Supporting Information

\title{
Photocatalytic Systems with Flavinium Salts: From Photolyase Models to Synthetic Tool for Cyclobutane Ring Opening
}

\author{
Tomáš Hartman and Radek Cibulka* \\ University of Chemistry and Technology, Prague Technická 5, 16628 Prague 6, \\ Czech Republic \\ *corresponding author: cibulkar@vscht.cz.
}

Table of Contents:

S1 General procedures and methods $\quad 2$

S2 Synthetic procedures and characterization of compounds $3 \quad 4$

S3 $[2+2]$ cycloreversion reactions catalyzed by $\mathbf{1}+\mathrm{TfOH}$

S4 $[2+2]$ cycloreversion reactions catalyzed by $\mathbf{2 c} \quad \mathbf{1 8}$

S5 Solvent screening in cycloreversion reactions 22

S6 UV-VIS and fluorescence spectra of the catalysts 23

S7 Fluorescence quenching of catalysts $\mathbf{1}$ and $\mathbf{2}$ with selected $\begin{array}{ll}\text { cyclobutanes } & \mathbf{2 7}\end{array}$

S8 Experimental setup for cycloreversion reaction under sunlight 30

S9 Effect of oxygen concentration on cycloreversion reaction with $\mathbf{2 c} \quad \mathbf{3 1}$

S10 Cycloreversion of isomeric pyrimidine dimers 32

S11 Stern-Volmer plots for quenching of $\mathbf{3 b}$-cycloreversion catalyzed by $\mathbf{2 c} \quad \mathbf{3 3}$

S12 Copy of NMR Spectra of compounds 3

S13 Copy of NMR Spectra of compounds 4

S14 Copy of NMR Spectra of compound 2c 54 
NMR spectra were recorded on a Varian Mercury Plus $300\left(299.97 \mathrm{MHz}\right.$ for ${ }^{1} \mathrm{H}, 75.44 \mathrm{MHz}$ for ${ }^{13} \mathrm{C}$, and $282.23 \mathrm{MHz}$ for ${ }^{19} \mathrm{~F}$ ) or Agilent 400-MR DDR2 (399.94 MHz for ${ }^{1} \mathrm{H}$ and 100.58 $\mathrm{MHz}$ for ${ }^{13} \mathrm{C}$ ) at $298 \mathrm{~K}$ unless otherwise indicated. Chemical shifts $\delta$ are given in ppm, using residual solvent or tetramethylsilane as an internal standard. Coupling constants $J$ are reported in Hz. UV-VIS spectra were recorded on a Varian Cary 50 spectrophotometer. Fluorescence spectra were recorded on Varian Cary Eclipse. High-resolution mass spectra were obtained on Q-Tof Micro (Waters), equipped with a quadrupole and TOF analyzers and MCP detector. TLC analyses were carried out on a DC Alufolien Kieselgel 60 F254 (Merck). Preparative column chromatography separations were performed on a silica gel Kieselgel 60 0.040-0.063 $\mathrm{mm}$ (Merck). Melting points were measured on a Boetius melting point apparatus and are uncorrected.

Electrochemical measurements were performed using a standard three-electrode system in a methrom type electrochemical cell with a glassy carbon working electrode, silver wire pseudo-reference electrode and a platinum wire auxiliary electrode. Cyclic voltammograms were collected by PGSTAT128N. The cyclic voltammetry measurements were carried out in acetonitrile containing a cyclobutane $3\left(c=1 \times 10^{-3} \mathrm{~mol} \mathrm{~L}^{-1}\right)$ and tetrabutylammonium hexafluorophosphate $\left(c=1 \times 10^{-1} \mathrm{~mol} \mathrm{~L}^{-1}\right)$ as supporting electrolyte under argon atmosphere. The scan rate was $100 \mathrm{mV} \mathrm{s}^{-1}$. The measured redox potentials were converted into values relative to SCE using standard redox couple $\mathrm{Fc}^{+} / \mathrm{Fc}$ as suggested by Addison and others: ${ }^{1}$ conversion of the measured values into those vs SCE involved subtraction of the difference between experimental $E_{1 / 2}$ values for standard redox couple $\mathrm{Fc}^{+} / \mathrm{Fc}$ measured after each experiment (relative to $\mathrm{Ag}$ wire) and $E_{1 / 2}$ value for standard redox couple $\mathrm{Fc}^{+} / \mathrm{Fc}$ measured against SCE at the same apparatus $(+0.381 \mathrm{~V}$, the value obtained as an average of 5 measurements).

Starting materials, reagents and substrates were obtained from commercial suppliers and used without further purification. The solvents were purified and dried using standard procedures. ${ }^{2}$ Riboflavin tetraacetate $(\mathbf{1}),{ }^{3}$ alloxazinium salts $2,{ }^{4}$ and isoalloxazinium salt $\mathbf{8}$

\footnotetext{
${ }^{1}$ a) Pavlishchuk, V. V.; Addison, A. W. Inorg. Chim. Acta 2000, 298, 97; b) Roth, H. G.; Romero, N. A.; Nicewicz, D. A. Synlett 2016, 27, 714.

2 D. D. Perrin, W. L. F. A. Purification of Laboratory Chemicals, 4th Ed.; Elsevier Science Ltd., Oxford, 1996.

${ }^{3}$ Neveselý, T.; Svobodová, E.; Chudoba, J.; Sikorski, M.; Cibulka, R. Adv. Synth. Catal. 2016, 358, 1654.

${ }^{4}$ Ménová, P.; Dvořáková, H.; Eigner, V.; Ludvík, J.; Cibulka, R. Adv. Synth. Catal. 2013, 355, 3451.
} 
$\left(\right.$ ref. $^{3}$ ) were prepared according to previously reported procedures. NMR and UV spectra are in agreement with previously reported data. ${ }^{2,3}$ 


\section{S2. Synthetic procedures and characterization of compounds 3}

$\left(6 \mathrm{a} R^{*}, 6 \mathrm{~b} R^{*}, 12 \mathrm{~b} R^{*}, 12 \mathrm{c} R^{*}\right)-5,8-D i m e t h y l-5,6 \mathrm{a}, 6 \mathrm{~b}, 8,12 \mathrm{~b}, 12 \mathrm{c}-h$ hexahydrocyclobuta-[1,2-c:4,3-c']diquinoline-6,7-dione (3a)

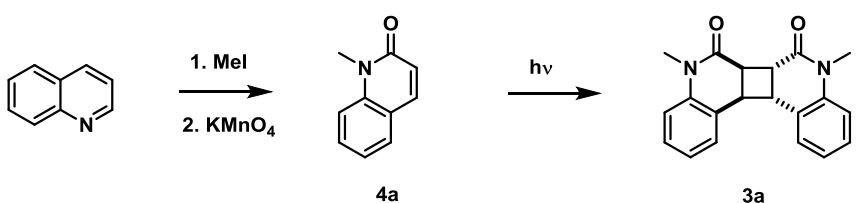

1-Methylquinolin-2(1H)-one (4a) Quinoline (15 g, $116 \mathrm{mmol})$ and iodomethane (16.48 g, 116 mmol) were mixed and allowed to stir/stand for 3 hours. Orange solids were dissolved in acetonitrile $(250 \mathrm{~mL})$ followed by addition of potassium permanganate $(45.9 \mathrm{~g}, 290 \mathrm{mmol})$ while stirring. Mixture color changed from violet to brown $\left(\mathrm{MnO}_{2}\right)$. Residual permanganate was decomposed with saturated solution of sodium sulfite. Resulting mixture was treated with $10 \% \mathrm{HCl}$ and extracted with chloroform. Chloroform solution was dried with magnesium sulfate and evaporated. Crude product was crystalized from ethanol to obtain 4a (9.8 g, 61.6 mmol, 53\% yield). M.p. $72-75^{\circ} \mathrm{C}$ (ref. $\left.55-76{ }^{\circ} \mathrm{C}\right) .{ }^{1} \mathrm{H}$ NMR $(400 \mathrm{MHz}$, Chloroform- $d$ ) $\delta 7.67$ $(\mathrm{d}, J=9.4 \mathrm{~Hz}, 1 \mathrm{H}), 7.61-7.53(\mathrm{~m}, 2 \mathrm{H}), 7.36(\mathrm{dd}, J=8.3,0.9 \mathrm{~Hz}, 1 \mathrm{H}), 7.29-7.18(\mathrm{~m}, 1 \mathrm{H})$, $6.71(\mathrm{~d}, J=9.5 \mathrm{~Hz}, 1 \mathrm{H}), 3.72(\mathrm{~s}, 3 \mathrm{H}) .{ }^{13} \mathrm{C}$ NMR $(75 \mathrm{MHz}$, Chloroform- $d$ ) $\delta 162.52,140.22$, $139.15,130.83,128.94,122.29,121.92,120.86,114.34,29.60$.

$\left(6 a R^{*}, 6 b R^{*}, 12 b R^{*}, 12 c R^{*}\right)-5,8-$ Dimethyl-5,6a,6b,8,12b,12c-hexahydrocyclobuta [1,2-c:4,3$c^{\prime}$ diquinoline-6,7-dione (3a) Solution of 1-methylquinolin-2(1H)-one (2 g, $\left.12.56 \mathrm{mmol}\right)$ in ethanol $(100 \mathrm{~mL})$ was irradiated in pyrex glassware for 10 hours using medium pressure mercury lamp $(125 \mathrm{~W})$. White precipitate was filtered off, washed with ethanol to give single isomer of 3a (1.5 g, $4.71 \mathrm{mmol}, 75 \%$ yield). M.p. $222-223{ }^{\circ} \mathrm{C}$ (ref. ${ }^{6} 220-222{ }^{\circ} \mathrm{C}$ ). ${ }^{1} \mathrm{H}$ NMR (400 MHz, Chloroform-d) $\delta 7.34-7.27$ (m, 2H), 7.07 - $6.98(\mathrm{~m}, 4 \mathrm{H}), 6.92$ (dd, $J=7.4,1.7$ $\mathrm{Hz}, 2 \mathrm{H}), 3.89-3.81(\mathrm{~m}, 2 \mathrm{H}), 3.79-3.71(\mathrm{~m}, 2 \mathrm{H}), 3.47(\mathrm{~s}, 6 \mathrm{H}) .{ }^{13} \mathrm{C}$ NMR $(101 \mathrm{MHz}$, Chloroform- $d$ ) $\delta 169.00,139.60,128.40,127.78,123.41,123.19,115.00,44.14,43.40,29.73$. HRMS (ESI+) Calcd for $\mathrm{C}_{20} \mathrm{H}_{18} \mathrm{O}_{2} \mathrm{~N}_{2}[\mathrm{M}+\mathrm{H}]^{+}:$320.14746; found: $\mathrm{m} / \mathrm{z}$ 320.14764. UV-VIS (acetonitrile): $259 \mathrm{~nm}$.

\footnotetext{
${ }^{5}$ Aksenov, A. V.; Smirnov, A. N.; Aksenov, N. A.; Aksenova, I. V.; Matheny, J. P.; Rubin, M. RSC Advances 2015, 5, 8647.

${ }^{6}$ Lewis, F. D.; Reddy, G. D.; Elbert, J. E.; Tillberg, B. E.; Meltzer, J. A.; Kojima, M. J. Org. Chem. 1991, 56, 5311.
} 
<smiles></smiles>

4b

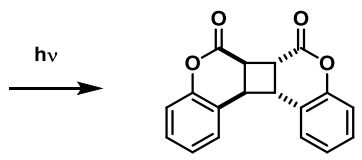

3b

Benzophenone $(0.156 \mathrm{~g}, 0.855 \mathrm{mmol})$ and coumarine $(2.5 \mathrm{~g}, 17.11 \mathrm{mmol})$ were dissolved in $150 \mathrm{~mL}$ of acetonitrile. Argon was bubbled through solution for 10 minutes followed by irradiation by medium pressure mercury lamp $(125 \mathrm{~W})$ in pyrex glassware. After 20 hours of irradiation, white precipitate was collected. Crystaline product was recrystalized from benzene to give $\mathbf{3 b}(2.375 \mathrm{~g}, 8.13 \mathrm{mmol}, 95 \%$ yield $)$ as a single isomer product. M.p. 176-178 ${ }^{\circ} \mathrm{C} .{ }^{1} \mathrm{H}$ NMR (400 MHz, Chloroform- $d$ ) $\delta 7.48-7.30(\mathrm{~m}, 2 \mathrm{H}), 7.23-7.07(\mathrm{~m}, 6 \mathrm{H}), 4.03$ $3.78(\mathrm{~m}, 4 \mathrm{H}) .{ }^{13} \mathrm{C}$ NMR (101 MHz, Chloroform- $d$ ) $\delta 165.98,151.18,129.70,127.78,125.39$, 120.24, 117.86, 43.86, 40.17. HRMS (ESI+) Calcd for $\mathrm{C}_{18} \mathrm{H}_{12} \mathrm{O}_{4}[\mathrm{M}+\mathrm{Na}]^{+}:$316.06613; found: $\mathrm{m} / \mathrm{z}$ 316.06641. UV-VIS (acetonitrile): 271, $280 \mathrm{~nm}$.

$\left(6 \mathrm{a} R^{*}, 6 \mathrm{~b} R^{*}, 12 \mathrm{~b} R^{*}, 12 \mathrm{c} R^{*}\right)-5,5,8,8-T e t r a m e t h y l-5,6 \mathrm{a}, 6 \mathrm{~b}, 8,12 \mathrm{~b}, 12 \mathrm{c}-h$ exahydrodibenzo[a,i]biphenylene-6,7-dione (3c)

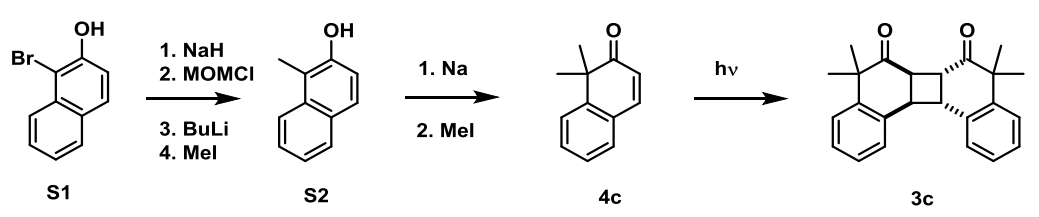

1-Methylnaphthalen-2-ol (S2) Sodium hydride (2.96 g, $74.0 \mathrm{mmol}$ ) was suspended in anhydrous THF $(100 \mathrm{ml})$. Mixture was cooled to $0{ }^{\circ} \mathrm{C}$ and 1-bromonaphthalen-2-ol $(15 \mathrm{~g}$, $67.2 \mathrm{mmol}$ ) was added portion wise. Mixture was stirred for one hour followed by addition of chloro(methoxy)methane $(7.66 \mathrm{~mL}, 101 \mathrm{mmol})$. Mixture was allowed to warm to room temperature and stirred for 2 hours. Reaction was quenched with ethanol and water. Mixture was extracted twice with ether. Ethereal part was washed with water, brine and dried using magnesium sulfate. Removal of solvent gave brown oil of 1-bromo-2(methoxymethoxy)naphthalene $(17.06 \mathrm{~g})$ which was used without further purification. ${ }^{1} \mathrm{H}$ NMR (400 MHz, Chloroform-d) $\delta 8.24$ (dd, $J=8.6,1.0 \mathrm{~Hz}, 1 \mathrm{H}), 7.84-7.76(\mathrm{~m}, 2 \mathrm{H}), 7.57$ (ddd, $J=8.5,6.9,1.3 \mathrm{~Hz}, 1 \mathrm{H}), 7.47-7.38(\mathrm{~m}, 2 \mathrm{H}), 5.36(\mathrm{~s}, 2 \mathrm{H}), 3.58(\mathrm{~s}, 3 \mathrm{H}) .{ }^{13} \mathrm{C}$ NMR $(101$ MHz, Chloroform-d) $\delta 151.7,133.1,130.5,128.9,128.1,127.7,126.4,124.9,117.0,110.5$, 95.6, 56.6. 1-Bromo-2-(methoxymethoxy)naphthalene (8 g, $29.9 \mathrm{mmol})$ was dissolved in THF $(100 \mathrm{~mL})$ under argon and cooled to $-70{ }^{\circ} \mathrm{C}$. While stirring, butyllithium $(13.18 \mathrm{~mL}, 32.9$ 
mmol) was added slowly. Mixture was allowed to stir for one hour at $-70{ }^{\circ} \mathrm{C}$, followed by addition of iodomethane $(4.66 \mathrm{~mL}, 74.9 \mathrm{mmol})$. Solution was allowed to warm to room temperature and stirred for 3 hours. Reaction was quenched by water $(10 \mathrm{~mL})$ and extracted with ether. Organic part was washed with water, brine and dried with magnesium sulfate. Ether was evaporated to give brown solid. Solids were dissolved in $\mathrm{MeOH}(60 \mathrm{~mL})$ and five drops of concentrated $\mathrm{HCl}$ were added. Solution was refluxed for 3 hours and evaporated to give S2 as yellow solid (2.73 g, $17.26 \mathrm{mmol}, 58 \%$ yield). ${ }^{1} \mathrm{H}$ NMR (400 MHz, Chloroform- $d$ ) $\delta 7.93(\mathrm{dd}, J=8.6,1.0 \mathrm{~Hz}, 1 \mathrm{H}), 7.80-7.76(\mathrm{~m}, 1 \mathrm{H}), 7.63(\mathrm{dd}, J=8.7,0.9 \mathrm{~Hz}, 1 \mathrm{H}), 7.51$ (ddd, $J=8.4,6.8,1.4 \mathrm{~Hz}, 1 \mathrm{H}), 7.35$ (ddd, $J=8.1,6.8,1.2 \mathrm{~Hz}, 1 \mathrm{H}), 7.07$ (d, $J=8.8 \mathrm{~Hz}, 1 \mathrm{H}$ ), $3.53(\mathrm{~s}, 1 \mathrm{H}), 2.55(\mathrm{~s}, 3 \mathrm{H}) .{ }^{13} \mathrm{C}$ NMR (101 MHz, Chloroform-d) $\delta 150.41,133.80,129.17$, $128.44,127.35,126.30,123.12-2$ signals, $117.55,115.18,10.47$.

1,1-Dimethylnaphthalen-2(1H)-one (4c) Sodium $(0.725 \mathrm{~g}, 31.5 \mathrm{mmol})$ was dissolved in $\mathrm{MeOH}(50 \mathrm{~mL})$. Solution of 1-methylnaphthalen-2-ol (S2, $4.75 \mathrm{~g}, 30.0 \mathrm{mmol})$ in $20 \mathrm{~mL}$ of methanol was added. Black solution was stirred overnight. Solvent was evaporated and solids were dried in vacuo. Brown solids were dissolved in iodomethane $(28.0 \mathrm{~mL}, 450 \mathrm{mmol})$ and refluxed for 20 hours. Iodomethane was evaporated, solids were dissolved in ether and water. Water was separated, extracted with ether. Ethereal part was washed with water, brine and dried with $\mathrm{MgSO}_{4}$. Crude oil was purified by column chromatography (hexane/ethyl acetate 10:1) to give $4 \mathrm{c}\left(2.2 \mathrm{~g}, 12.77 \mathrm{mmol}, 43 \%\right.$ yield) as an oil. ${ }^{1} \mathrm{H}$ NMR (400 MHz, Chloroform- $d$ ) $\delta 7.49-7.38(\mathrm{~m}, 3 \mathrm{H}), 7.35-7.27(\mathrm{~m}, 2 \mathrm{H}), 6.17(\mathrm{~d}, J=9.8 \mathrm{~Hz}, 1 \mathrm{H}), 1.47(\mathrm{~s}, 6 \mathrm{H}) .{ }^{13} \mathrm{C} \mathrm{NMR}$ (101 MHz, Chloroform-d) $\delta$ 204.55, 147.65, 144.79, 130.05, 129.44, 128.68, 126.67, 126.23, $124.50,47.44,27.86$.

$\left(6 a R^{*}, 6 b R^{*}, 12 b R^{*}, 12 c R^{*}\right)-5,5,8,8$-Tetramethyl-5,6a,6b, 8,12b,12c-hexahydrodibenzo-[a,i]biphenylene-6,7-dione (3c) 1,1-Dimethylnaphthalen-2(1H)-one (4c, $400 \mathrm{mg}, 2.323$ mmol) was dissolved in ethanol $(5 \mathrm{~mL})$ and solution was irradiated in pyrex glassware for 20 hrs using medium pressure mercury lamp $(125 \mathrm{~W})$. White precipitate was filtered off, washed with ethanol to give single isomer of 3c (364 mg, $1.057 \mathrm{mmol}, 91 \%$ yield). M.p. 192-194 ${ }^{\circ} \mathrm{C}$. ${ }^{1} \mathrm{H}$ NMR (400 MHz, Chloroform- $d$ ) $\delta 7.54-7.28(\mathrm{~m}, 8 \mathrm{H}), 4.21-4.11(\mathrm{~m}, 2 \mathrm{H}), 3.81(\mathrm{dt}, J=$ 8.4, $1.4 \mathrm{~Hz}, 2 \mathrm{H}), 1.58(\mathrm{~s}, 6 \mathrm{H}), 1.38(\mathrm{~s}, 6 \mathrm{H}) .{ }^{13} \mathrm{C}$ NMR (101 MHz, Chloroform-d) $\delta 208.94$, 139.99, 132.63, 123.97, 123.47, 123.30, 121.50, 44.63, 43.54, 38.14, 25.04, 17.23. HRMS (ESI+) calcd for $\mathrm{C}_{24} \mathrm{H}_{24} \mathrm{O}_{2}[\mathrm{M}+\mathrm{Na}]^{+}: 368.17021$; found: $368.17052 \mathrm{~m} / \mathrm{z}$. UV-VIS (acetonitrile): 219, $267 \mathrm{~nm}$. 


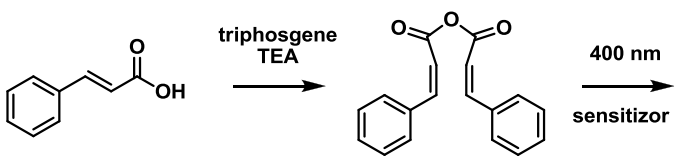

S3

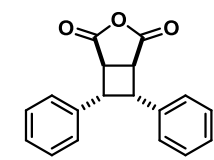

3d

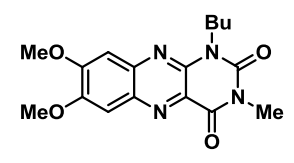

sensitizor

Cinnamic anhydride (4d) Cinnamic acid (10 g, $67.5 \mathrm{mmol})$ and triethylamine $(6.83 \mathrm{~g}, 67.5$ mmol) were stirred in ethyl acetate $(50 \mathrm{~mL})$ and cooled to $0{ }^{\circ} \mathrm{C}$. Bis(trichloromethyl) carbonate (3.91 g, $11.47 \mathrm{mmol}$ ) was added portion wise keeping the temperature below $10{ }^{\circ} \mathrm{C}$. Reaction was stirred for one hour, filtered and washed with ethyl acetate. Filtrate was evaporated to give white crystals of $4 \mathbf{d}$ (16.7 g, $60.0 \mathrm{mmol}, 89 \%$ yield). M.p. $134-135{ }^{\circ} \mathrm{C}$ (ref. $\left.{ }^{7} 134-136{ }^{\circ} \mathrm{C}\right) .{ }^{1} \mathrm{H}$ NMR $(300 \mathrm{MHz}$, Chloroform- $d) \delta 7.86(\mathrm{~d}, J=15.9 \mathrm{~Hz}, 2 \mathrm{H}), 7.62-$ $7.55(\mathrm{~m}, 4 \mathrm{H}), 7.47-7.39(\mathrm{~m}, 6 \mathrm{H}), 6.53(\mathrm{dd}, J=15.9,0.8 \mathrm{~Hz}, 2 \mathrm{H}) .{ }^{13} \mathrm{C}$ NMR $(75 \mathrm{MHz}$, Chloroform- $d$ ) $\delta 162.71,148.89,133.94,131.51,129.31,128.81,116.97$.

$\left(1 R^{*}, 5 S^{*}, 6 R^{*}, 7 S^{*}\right)-6,7-$ Diphenyl-3-oxabicyclo[3.2.0]heptane-2,4-dione (3d) Cinnamic anhydride (2 g, $7.19 \mathrm{mmol})$ and 1-butyl-7,8-dimethoxy-3-methylbenzo[g]pteridine2,4(1H,3H)-dione ${ }^{8} \mathbf{S 9}(0.247 \mathrm{~g}, 0.719 \mathrm{mmol})$ in acetonitrile $(50 \mathrm{~mL})$ were irradiated with 400 $\mathrm{nm}$ LED lamp for 3 hours. Solvent was evaporated to give yellow foam of 3d (1.92 g, 6.90 mmol, 96\% yield). ${ }^{1} \mathrm{H}$ NMR (400 MHz, Chloroform- $d$ ) $\delta 7.22-7.05(\mathrm{~m}, 6 \mathrm{H}), 6.95-6.84(\mathrm{~m}$, $4 \mathrm{H}), 4.44-4.36(\mathrm{~m}, 2 \mathrm{H}), 3.99-3.91(\mathrm{~m}, 2 \mathrm{H}) .{ }^{13} \mathrm{C}$ NMR (101 MHz, Chloroform- $d$ ) $\delta 172.18$, 136.32, 128.31, 127.63, 127.19, 46.94, 42.94. HRMS (ESI+) calcd for $\mathrm{C}_{18} \mathrm{H}_{15} \mathrm{O}_{3}[\mathrm{M}+\mathrm{H}]^{+}$: 279.10216; found: $279.10174 \mathrm{~m} / \mathrm{z}$. UV-VIS (acetonitrile): 217, $258 \mathrm{~nm}$.

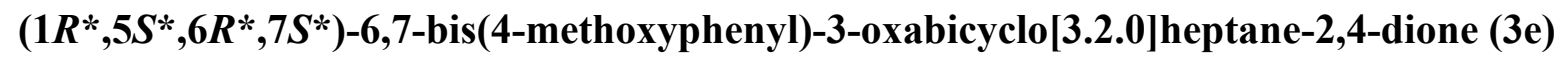

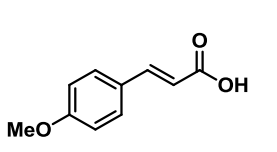

S4

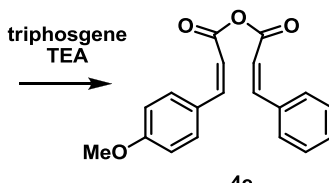

$4 \mathrm{e}$

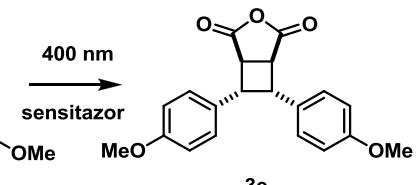

$3 e$

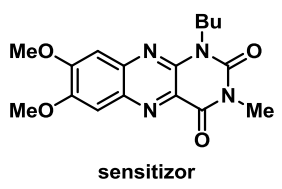

sensitizor

(E)-3-(4-methoxyphenyl)acrylic anhydride (4e) (E)-3-(4-Methoxyphenyl)acrylic acid (8.6 g, $48.3 \mathrm{mmol})$ and triethylamine $(6.94 \mathrm{~mL}, 48.3 \mathrm{mmol})$ were stirred in ethyl acetate $(50 \mathrm{~mL})$ and cooled to $0{ }^{\circ} \mathrm{C}$. Bis(trichloromethyl) carbonate $(2.435 \mathrm{~g}, 8.20 \mathrm{mmol})$ was added portion wise keeping the temperature below $10{ }^{\circ} \mathrm{C}$. Reaction was stirred for one hour, filtered and washed with ethyl acetate. Filtrate was evaporated to give white foam of $4 \mathbf{e}(6.61 \mathrm{~g}, 19.55$

\footnotetext{
${ }^{7}$ Konieczynska, M. D.; Dai, C.; Stephenson, C. R. J. Org. Biomol. Chem. 2012, 10, 4509.

${ }^{8}$ Mojr, V.; Svobodova, E.; Strakova, K.; Nevesely, T.; Chudoba, J.; Dvorakova, H.; Cibulka, R. Chem. Commun. 2015, 51, 12036.
} 
mmol, 81\% yield). ${ }^{1} \mathrm{H}$ NMR (400 MHz, Chloroform- $d$ ) $\delta 7.80(\mathrm{~d}, J=15.9 \mathrm{~Hz}, 2 \mathrm{H}), 7.58-$ $7.50(\mathrm{~m}, 4 \mathrm{H}), 6.97-6.91(\mathrm{~m}, 4 \mathrm{H}), 6.39(\mathrm{~d}, J=15.8 \mathrm{~Hz}, 2 \mathrm{H}), 3.86(\mathrm{~s}, 6 \mathrm{H}) .{ }^{13} \mathrm{C} \mathrm{NMR}(101$ MHz, Chloroform- $d$ ) $\delta 163.01,162.15,148.24,130.40,126.52,114.50,114.15,55.42$ (d, $J=$ $4.5 \mathrm{~Hz})$.

$\left(1 R^{*}, 5 S^{*}, 6 R^{*}, 7 S^{*}\right)-6,7-b i s(4-m e t h o x y p h e n y l)-3-o x a b i c y c l o[3.2 .0]$ heptane-2,4-dione (3e)

1-Butyl-7,8-dimethoxy-3-methylbenzo[g]pteridine-2,4(1H,3H)-dione ${ }^{8}(0.051 \mathrm{~g}, 0.148 \mathrm{mmol})$ and (E)-3-(4-methoxyphenyl)acrylic anhydride (4e, $1 \mathrm{~g}, 2.96 \mathrm{mmol})$ were dissolved in acetonitrile $(20 \mathrm{~mL})$. Solution was irradiated for 2 hours with LED (400 nm). Solvent was evaporated, crude product was purified by column chromatography (hexane/ethyl acetate 5:1) to gain $3 \mathrm{e}$ (460 mg, $1.360 \mathrm{mmol}, 46 \%$ yield) as an oil. ${ }^{1} \mathrm{H}$ NMR (400 MHz, Chloroform- $d$ ) $\delta$ $7.26(\mathrm{~s}, 1 \mathrm{H}), 6.88-6.77(\mathrm{~m}, 4 \mathrm{H}), 6.66(\mathrm{~d}, J=8.7 \mathrm{~Hz}, 4 \mathrm{H}), 4.35-4.28(\mathrm{~m}, 2 \mathrm{H}), 3.81(\mathrm{dd}, J=$ 5.5, $1.8 \mathrm{~Hz}, 2 \mathrm{H}), 3.70$ (s, 6H). ${ }^{13} \mathrm{C}$ NMR (101 MHz, Chloroform-d) $\delta 179.27,158.08,130.31$, 128.80, 113.52, 55.11, 44.11, 43.86. HRMS (ESI+) calcd for $\mathrm{C}_{20} \mathrm{H}_{18} \mathrm{O}_{5}[\mathrm{M}+\mathrm{H}]^{+}:$339.11542; found: m/z 339.11483. UV-VIS (acetonitrile): 228, $278 \mathrm{~nm}$.

\section{$\left(4 \mathrm{a} R^{*}, 4 \mathrm{~b} S^{*}, 8 \mathrm{a} S^{*}, 8 \mathrm{~b} R^{*}\right)-1,3,6,8-$ Tetramethylhexahydrocyclobuta[1,2-d:4,3-}

$d^{\prime}$ ]dipyrimidine-2,4,5,7(3H,6H)-tetraone (3f)

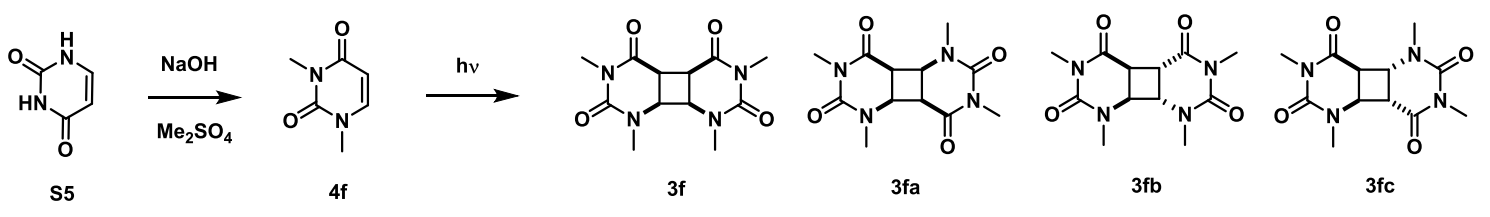

1,3-Dimethylpyrimidine-2,4(1H,3H)-dione (4f) Pyrimidine-2,4(1H,3H)-dione (15 g, 134 mmol) and sodium hydroxide $(12.85 \mathrm{~g}, 321 \mathrm{mmol})$ were dissolved in water $(150 \mathrm{~mL})$. Mixture was stirred for one hour followed by addition of dimethyl sulfate $(30.5 \mathrm{~mL}, 321$ mmol). Solution was heated to $60{ }^{\circ} \mathrm{C}$ overnight. Water was evaporated, white solids were dissolved in dichloromethane, washed twice with water, brine and organic part was dried using magnesium sulfate. After solvent removal, white crystals were dried to give $\mathbf{4 f}$ (14.5 g, 103 mmol, 77\% yield). M.p. 118-121 ${ }^{\circ} \mathrm{C}$ (ref. $\left.{ }^{9} \quad 122-124{ }^{\circ} \mathrm{C}\right) .{ }^{1} \mathrm{H}$ NMR (400 MHz, Chloroform- $d$ ) $\delta 7.12$ (d, $J=7.9 \mathrm{~Hz}, 1 \mathrm{H}), 5.73$ (d, $J=7.8 \mathrm{~Hz}, 1 \mathrm{H}), 3.39$ (s, 3H), 3.34 (s, 3H). ${ }^{13} \mathrm{C}$ NMR (101 MHz, Chloroform- $d$ ) $\delta$ 163.35, 151.92, 142.66, 101.38, 37.02, 27.75.

(4aR*,4bS*,8aS*,8bR*)-1,3,6,8-Tetramethylhexahydrocyclobuta[1,2-d:4,3-d'] dipyrimidine2,4,5,7(3H,6H)-tetraone (3f) 1,3-Dimethylpyrimidine-2,4(1H,3H)-dione $\quad(\mathbf{4 f}, \quad 10$ g, 71.4 mmol) was irradiated in acetone $(200 \mathrm{~mL})$ using quartz glassware and medium pressure

\footnotetext{
${ }^{9}$ Kolonko, K. J.; Shapiro, R. H.; Barkley, R. M.; Sievers, R. E. J. Org. Chem. 1979, 44, 3769.
} 
mercury lamp (125 W) for 5 hours. Solvent was evaporated, solids crystalized from ethanol to give mixture of four isomers of uracil dimer in ratio $\mathbf{3 f} / \mathbf{3 f a} / \mathbf{3 f b} / \mathbf{3 f c} 50: 30: 15: 5$. Mixture was separated by column chromatography on silica gel (hexan/acetone 6:4) as starting eluent and long column $1 \mathrm{~g} / 150 \mathrm{~g}$ of silica. After elution of first isomer $\mathbf{3 f c}$, eluent changed to hexane/acetone 4:6, to separate second $\mathbf{3 f b}$ and third isomer $\mathbf{3 f a}$. Pure acetone was used to elute fourth isomer 3f. Data for 3f: M.p. $245-247{ }^{\circ} \mathrm{C}$. (ref. $\left.{ }^{10} 241-243{ }^{\circ} \mathrm{C}\right) .{ }^{1} \mathrm{H}$ NMR $(400 \mathrm{MHz}$, Chloroform-d) $\delta 4.07$ (d, J = 9.8 Hz, 2H), 3.78 (d, J = 9.9 Hz, 2H), 3.15 (s, 6H), 2.99 (s, 6H). ${ }^{13} \mathrm{C}$ NMR of $3 \mathbf{f}$ (101 MHz, Chloroform-d) $\delta$ 165.69, 152.63. UV-VIS (acetonitrile): $225 \mathrm{~nm}$.

Data for 3fa: ${ }^{1} \mathrm{H}$ NMR (400 MHz, Chloroform- $d$ ) $\delta 4.09(\mathrm{t}, J=8.5 \mathrm{~Hz}, 2 \mathrm{H}), 3.76(\mathrm{t}, J=8.5$ $\mathrm{Hz}, 2 \mathrm{H}), 3.12$ (s, 6H), 3.10 (s, 6H). ${ }^{13} \mathrm{C}$ NMR (101 MHz, Chloroform- $d$ ) $\delta 165.94,152.03$, 49.16, 45.22, 35.51, 27.62 .

Data for 3fb: ${ }^{1} \mathrm{H}$ NMR (400 MHz, Chloroform- $d$ ) $\delta 3.87$ (d, $\left.J=8.4 \mathrm{~Hz}, 2 \mathrm{H}\right), 3.61$ (d, $J=8.4$ $\mathrm{Hz}, 2 \mathrm{H}), 3.24$ (s, 6H), 3.06 (s, 6H). ${ }^{13} \mathrm{C}$ NMR (101 MHz, Chloroform- $d$ ) $\delta 168.07,151.74$, 59.28, 39.13, 35.09, 28.27 .

Data for 3fc: ${ }^{1} \mathrm{H}$ NMR (400 MHz, Chloroform-d) $\delta 4.16-4.06(\mathrm{~m}, 2 \mathrm{H}), 3.57-3.45(\mathrm{~m}, 2 \mathrm{H})$, $3.25(\mathrm{~s}, 6 \mathrm{H}), 3.09$ (s, 6H). ${ }^{13} \mathrm{C} \mathrm{NMR}(101 \mathrm{MHz}$, Chloroform- $d$ ) $\delta$ 166.95, 151.38, 53.41, $44.33,33.68,27.93$.

\section{$\left(1 S^{*}, 6 S^{*}\right)-2,4,7,7,8,8-H e x a m e t h y l-2,4-d i a z a b i c y c l o[4.2 .0]$ octane-3,5-dione (3g)}

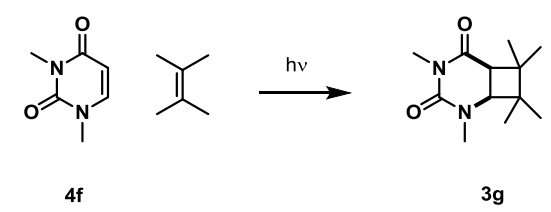

1,3-Dimethylpyrimidine-2,4(1H,3H)-dione (1.5 g, $10.70 \mathrm{mmol})$ was dissolved in acetone (150 $\mathrm{mL}$ ) and 2,3-dimethylbut-2-ene (12.87 mL, $107 \mathrm{mmol})$ was added. Mixture was bubbled with argon for 15 minutes. Solution was irradiated in quartz glassware by medium pressure mercury lamp $(125 \mathrm{~W})$. After 8 hours of irradiation, solvent was removed and oily residual was purified by column chromatography (hexane/acetone $7 / 3$ ) to give colorless oil of $\mathbf{3 g}$ (1.1 g, $4.90 \mathrm{mmol}, 46 \%$ yield). ${ }^{1} \mathrm{H}$ NMR (400 MHz, Chloroform- $d$ ) $\delta 3.57$ (d, $\left.J=10.1 \mathrm{~Hz}, 1 \mathrm{H}\right)$, $3.20(\mathrm{~s}, 3 \mathrm{H}), 3.01-2.93(\mathrm{~m}, 4 \mathrm{H}), 1.22(\mathrm{~s}, 3 \mathrm{H}), 1.10(\mathrm{~s}, 3 \mathrm{H}), 0.97(\mathrm{~s}, 3 \mathrm{H}), 0.92(\mathrm{~s}, 3 \mathrm{H}) .{ }^{13} \mathrm{C}$ NMR (101 MHz, Chloroform-d) $\delta$ 169.43, 153.02, 57.42, 45.25, 44.95, 41.86, 35.87, 27.28, 27.02, 24.51, 20.27, 19.19. HRMS (ESI+) Calcd for $\mathrm{C}_{12} \mathrm{H}_{20} \mathrm{O}_{2} \mathrm{~N}_{2}[\mathrm{M}+\mathrm{H}]^{+}:$225.15975; found: $\mathrm{m} / \mathrm{z} 225.15947$. UV-VIS (acetonitrile): $<225 \mathrm{~nm}$.

\footnotetext{
${ }^{10}$ Gorelic, L. S.; Lisagor, P.; Yang, N. C. Photochem. Photobiol. 1972, 16, 465.
} 


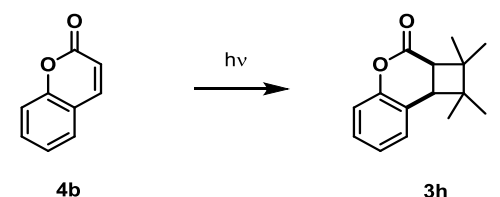

(2aR*,8bS*)-1,1,2,2-Tetramethyl-1,2,2a,8b-tetrahydro-3H-cyclobuta[c]chromen-3-one (3h) 2H-Chromen-2-one (1 g, $6.84 \mathrm{mmol})$ and benzophenone $(0.125 \mathrm{~g}, 0.684 \mathrm{mmol})$ were dissolved in dioxane $(150 \mathrm{~mL})$ and argon was bubbled through solution for 10 minutes. Then 2,3-dimethylbut-2-ene (2.88 g, $34.2 \mathrm{mmol})$ was added and mixture was irradiated for 6 hours in pyrex glassware using medium pressure mercury lamp (125 W). Solvent was evaporated, crude product purified by column chromatography (hexane/ethyl acetate 10:1) and crystalized from hexane to give $\mathbf{3 h}$ (500 mg, $2.171 \mathrm{mmol}, 32 \%$ yield). M.p. $>320{ }^{\circ} \mathrm{C}$. ${ }^{1} \mathrm{H}$ NMR (400 $\mathrm{MHz}$, Chloroform-d) $\delta 7.20$ (ddd, J = 7.9, 1.7, 0.7 Hz, 1H), 7.07 (td, J = 7.4, $1.2 \mathrm{~Hz}, 1 \mathrm{H}), 7.01$ - 6.97 (m, 2H), 3.37 (d, J = 9.7 Hz, 2H), 3.19 (d, J = 9.7 Hz, 2H), 1.26 (s, 3H), 1.21 (s, 3H), $1.01(\mathrm{~s}, 3 \mathrm{H}), 0.75$ (s, 3H). ${ }^{13} \mathrm{C}$ NMR (101 MHz, Chloroform-d) $\delta 167.24,151.62,129.19$, 128.18, 124.15, 120.55, 117.10, 45.22, 44.52, 43.07, 41.45, 26.31, 26.01, 21.34, 20.86. HRMS (ESI+) Calcd for $\mathrm{C}_{15} \mathrm{H}_{18} \mathrm{O}_{2}[\mathrm{M}+\mathrm{Na}]^{+}$: 253.11990; found: $\mathrm{m} / \mathrm{z}$ 253.12021. UV-VIS (acetonitrile): 271, $279 \mathrm{~nm}$.

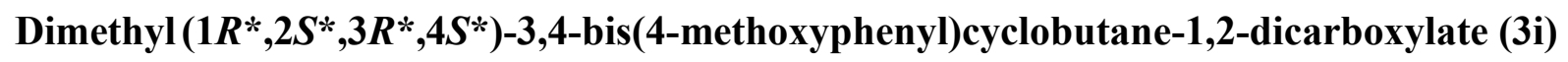

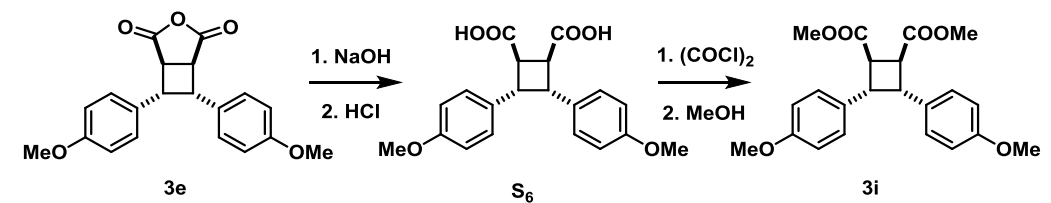

Dimethyl (1R*,2S*,3R*,4S*)-3,4-bis(4-methoxyphenyl)cyclobutane-1,2-dicarboxylate (3i)

3e $(850 \mathrm{mg}, 2.51 \mathrm{mmol})$ was dissolved in $\mathrm{MeOH}(5 \mathrm{~mL})$. Solution of potassium hydroxide (282 mg, $5.02 \mathrm{mmol})$ in $\mathrm{MeOH}(5 \mathrm{~mL})$ was added. White precipitate formed immediately. Solids were separated, dissolved in water $(2 \mathrm{~mL})$ and dichloromethane $(2 \mathrm{~mL})$. Solution was acidified using concentrated $\mathrm{HCl}$. Mixture was stirred for 20 minutes, organic part was separated, water phase was washed with dichloromethane. Organic part was dried with magnesium sulfate and evaporated to give crude acid $\mathbf{S 6}(850 \mathrm{mg})$ which was used in following step without purification. S6 $(480 \mathrm{mg}, 1.347 \mathrm{mmol})$ was suspended in dichloromethane $(3 \mathrm{~mL})$ and oxalyl dichloride $(289 \mu 1,3.37 \mathrm{mmol})$ was added. Reaction was started with 3 drops of DMF. When solution became homogenous, solution of methanol (272 
$\mu 1,6.73 \mathrm{mmol})$ in dichloromethane $(3 \mathrm{~mL})$ was added dropwise. Reaction was quenched by water, organic part separated, washed with saturated $\mathrm{NaHCO}_{3}$, brine and dried with magnesium sulfate. Solvent evaporation gave orange oil, which was further purified by column chromatography (hexane/ethyl acetate 5:1). 3i (310 mg, $0.806 \mathrm{mmol}, 60 \%$ yield) was obtained as colorless oil. ${ }^{1} \mathrm{H}$ NMR (400 MHz, Chloroform- $d$ ) $\delta 6.83(\mathrm{~d}, J=8.7 \mathrm{~Hz}, 4 \mathrm{H}), 6.66$ (d, $J=8.8 \mathrm{~Hz}, 4 \mathrm{H}), 4.33-4.27(\mathrm{~m}, 2 \mathrm{H}), 3.76(\mathrm{~d}, J=6.0 \mathrm{~Hz}, 2 \mathrm{H}), 3.74(\mathrm{~s}, 6 \mathrm{H}), 3.71(\mathrm{~s}, 6 \mathrm{H})$. ${ }^{13} \mathrm{C}$ NMR (101 MHz, Chloroform-d) $\delta$ 173.04, 157.98, 130.67, 128.81, 113.43, 55.11, 52.11, 44.32, 43.55. HRMS (ESI+) Calcd for $\mathrm{C}_{22} \mathrm{H}_{24} \mathrm{O}_{6}[\mathrm{M}+\mathrm{Na}]^{+}: 407.14651$; found: $\mathrm{m} / \mathrm{z} 407.14693$. UV-VIS (acetonitrile): 228, $278 \mathrm{~nm}$.

\section{Dimethyl $\left(1 R^{*}, 2 R^{*}, 3 S^{*}, 4 S^{*}\right)-3,4-$-bis(4-methoxyphenyl)cyclobutane-1,2-dicarboxylate (3j)}

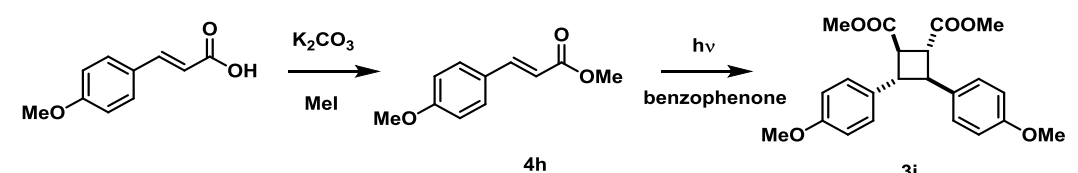

$4 h$

3j

Methyl (E)-3-(4-methoxyphenyl)acrylate (4h) To a suspension of (E)-3-(4methoxyphenyl)acrylic acid (18.5 g, $104 \mathrm{mmol})$ and potassium carbonate $(28.7 \mathrm{~g}, 208 \mathrm{mmol})$ in acetone $(300 \mathrm{~mL})$ dimethyl sulfate $(19.69 \mathrm{~mL}, 208 \mathrm{mmol})$ was added. Mixture was heated to $50{ }^{\circ} \mathrm{C}$ overnight. Solids were filtered off, washed with ether. Filtrate was evaporated and crude product crystalized from diisopropyl ether to afford $\mathbf{4 h}$ (14 g, $72.8 \mathrm{mmol}, 70 \%$ yield). M.p. 89-92 ${ }^{\circ} \mathrm{C} .{ }^{1} \mathrm{H}$ NMR (400 MHz, Chloroform-d) $\delta 7.65(\mathrm{~d}, \mathrm{~J}=16.0 \mathrm{~Hz}, 1 \mathrm{H}), 7.55-7.42$ $(\mathrm{m}, 2 \mathrm{H}), 6.90(\mathrm{~d}, \mathrm{~J}=8.8 \mathrm{~Hz}, 2 \mathrm{H}), 6.31(\mathrm{~d}, \mathrm{~J}=16.0 \mathrm{~Hz}, 1 \mathrm{H}), 3.83(\mathrm{~s}, 3 \mathrm{H}), 3.79(\mathrm{~s}, 3 \mathrm{H}) .{ }^{13} \mathrm{C}$ NMR (101 MHz, Chloroform-d) $\delta 167.75,161.36,144.51,129.70,127.09,115.24,114.30$, $55.36,51.57$.

Dimethyl (1R*,2R*,3S*,4S*)-3,4-bis(4-methoxyphenyl)cyclobutane-1,2-dicarboxylate (3j) Benzophenone $(0.190 \mathrm{~g}, 1.041 \mathrm{mmol})$ and $4 \mathrm{~h}(1 \mathrm{~g}, 5.20 \mathrm{mmol})$ were dissolved in benzene $(100 \mathrm{~mL})$. Argon was bubbled through mixture for $10 \mathrm{mins}$ followed by 20 hours of irradiation using medium pressure mercury arc lamp (125 W). Solvent removal and column chromatography (hexane/ethyl acetate 10:1) afforded starting material and mixture of $\mathbf{3 i}$ and 3j. 3j (250 mg, $0.650 \mathrm{mmol}, 25 \%$ yield) was obtained as an oil. ${ }^{1} \mathrm{H}$ NMR (400 MHz, Chloroform-d) $\delta 7.20$ (d, J = 8.7 Hz, 4H), 6.85 (d, J = 8.7 Hz, 4H), 3.79 (s, 3H), 3.73 (s, 3H), $3.59(\mathrm{~d}, \mathrm{~J}=9.6 \mathrm{~Hz}, 2 \mathrm{H}), 3.42(\mathrm{~d}, \mathrm{~J}=9.6 \mathrm{~Hz}, 2 \mathrm{H}) .{ }^{13} \mathrm{C}$ NMR (101 MHz, Chloroform-d) $\delta$ 173.07, 158.66, 133.07, 127.90, 113.97, 55.26, 52.15, 47.30, 44.55. HRMS (ESI+) Calcd for $\mathrm{C}_{22} \mathrm{H}_{24} \mathrm{O}_{6}[\mathrm{M}+\mathrm{Na}]^{+}:$407.14651; found: m/z 407.14699. UV-VIS (acetonitrile): 280, $286 \mathrm{~nm}$. 
$\left(1 R^{*}, 2 R^{*}, 3 S^{*}, 4 S^{*}\right)-1,2,3,4-T e t r a k i s(4-m e t h o x y p h e n y l) c y c l o b u t a n e ~(3 k)$

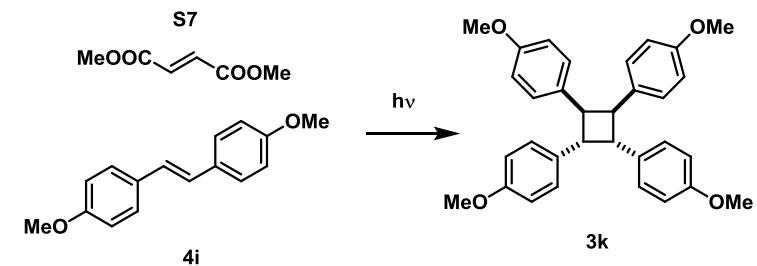

(Z)-1,2-Bis(4-methoxyphenyl)ethene (4i) (4-Methoxybenzyl)triphenylphosphonium bromide (37.2 $\mathrm{g}, 80 \mathrm{mmol})$ was suspended in toluene $(200 \mathrm{~mL})$ and potassium 2methylpropan-2-olate $(10.81 \mathrm{~g}, 96 \mathrm{mmol})$ was added. Mixture was stirred at room temperature for 3 hours, followed by addition of 4-methoxybenzaldehyde (14.65 mL, $120 \mathrm{mmol})$. Solution was stirred overnight. Solvent was evaporated, hexane was added to precipitate triphenylphosphine oxide. Suspension was filtered and evaporated to give crude product, which was used in following isomeration reaction. Crude (Z)-1,2-bis(4-methoxyphenyl)ethene (4 g, $16.65 \mathrm{mmol})$ was dissolved in THF $(50 \mathrm{~mL})$ and 1,2-diphenyldisulfane $(0.727 \mathrm{~g}$, $3.33 \mathrm{mmol}$ ) was added. Mixture was refluxed for 6 hours and evaporated. Crude product was purified by column chromatography (hexane/ethyl acetate 10:1). Pure 4i (3 g, 12.48 mmol, $75 \%$ yield) was obtained as white crystals. M.p. 211-214 ${ }^{\circ} \mathrm{C} .{ }^{1} \mathrm{H}$ NMR $(400 \mathrm{MHz}$, Chloroform-d) $\delta 7.43$ (d, J = 8.7 Hz, 4H), 6.93 (s, 2H), 6.89 (d, J = 8.7 Hz, 4H), 3.83 (s, 6H). ${ }^{13} \mathrm{C}$ NMR (101 MHz, Chloroform-d) $\delta$ 158.96, 130.44, 127.39, 126.14, 114.07, 55.31.

$\left(1 R^{*}, 2 R^{*}, 3 S^{*}, 4 S^{*}\right)-1,2,3,4-$ Tetrakis(4-methoxyphenyl)cyclobutane (3k) (E)-1,2-Bis(4methoxyphenyl)ethene $(800 \mathrm{mg}, 3.33 \mathrm{mmol})$ in benzene was irradiated in pyrex glassware using medium pressure mercury arc lamp (125 W) for 20 hours. Solvent was evaporated and crude yellow oil was purified by column chromatography (hexane/ethyl acetate 10:1). Beside starting material, 3k (300 mg, $0.624 \mathrm{mmol}, 19 \%$ yield) was obtained as an oil. ${ }^{1} \mathrm{H}$ NMR (400 MHz, Chloroform-d) $\delta 7.01$ (d, J = 8.7 Hz, 8H), 6.70 (d, J = 8.7 Hz, 8H), 4.30 (s, 4H), 3.72 (s, 12H). ${ }^{13} \mathrm{C}$ NMR (101 MHz, Chloroform-d) $\delta$ 157.60, 133.10, 129.08, 113.34, 55.10, 47.13. HRMS (ESI+) Calcd for $\mathrm{C}_{22} \mathrm{H}_{24} \mathrm{O}_{6}[\mathrm{M}+\mathrm{Na}]^{+}$: 503.21687; found: $\mathrm{m} / \mathrm{z}$ 503.21599. UV-VIS (acetonitrile): 281, $287 \mathrm{~nm}$.

Dimethyl $\left(1 R^{*}, 2 S^{*}, 3 R^{*}, 4 S^{*}\right)-3,4-$-bis(furan-2-yl)cyclobutane-1,2-dicarboxylate (3I)
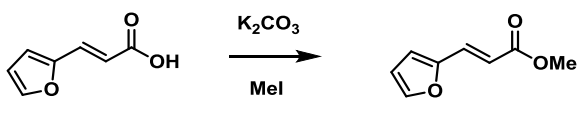

$4 \mathbf{j}$
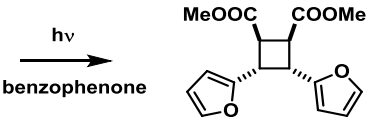

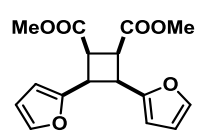

$31 a$ 
and potassium carbonate $(20.01 \mathrm{~g}, 145 \mathrm{mmol})$ were suspended in acetone $(150 \mathrm{~mL})$ and dimethyl sulfate $(13.73 \mathrm{~mL}, 145 \mathrm{mmol})$ was added. Mixture was heated to $50^{\circ} \mathrm{C}$ overnight. Solids were filtered off, filtrate evaporated. Residual was dissolved in dichloromethane and washed with water, brine and evaporated to give $4 \mathbf{j}$ ( $8.5 \mathrm{~g}, 55.9 \mathrm{mmol}$, 77\% yield) as yellow oil. ${ }^{1} \mathrm{H}$ NMR (400 MHz, Chloroform-d) $\delta 7.48$ (dt, J = 1.8, $0.6 \mathrm{~Hz}, 1 \mathrm{H}$ ), 7.43 (d, J = $15.7 \mathrm{~Hz}$, 1H), $7.26(\mathrm{~s}, 0 \mathrm{H}), 6.61(\mathrm{dd}, \mathrm{J}=3.4,0.6 \mathrm{~Hz}, 1 \mathrm{H}), 6.46(\mathrm{dd}, \mathrm{J}=3.4,1.8 \mathrm{~Hz}, 1 \mathrm{H}), 6.31(\mathrm{~d}, \mathrm{~J}=$ $15.7 \mathrm{~Hz}, 1 \mathrm{H}), 3.78(\mathrm{~s}, 3 \mathrm{H}) .{ }^{13} \mathrm{C}$ NMR (101 MHz, Chloroform-d) $\delta$ 167.46, 150.85, 144.71, $131.17,115.43,114.76,112.24,51.64$.

Dimethyl (1 $\left.R^{*}, 2 S^{*}, 3 R^{*}, 4 S^{*}\right)$-3,4-di(furan-2-yl)cyclobutane-1,2-dicarboxylate (3l) Methyl (E)-3-(furan-2-yl)acrylate $(4 \mathbf{j}, 1.5 \mathrm{~g}, 9.86 \mathrm{mmol})$ and benzophenone $(0.15 \mathrm{~g}, 0.823 \mathrm{mmol})$ were dissolved in acetonitrile $(150 \mathrm{~mL})$. Solution was irradiated in pyrex glassware using medium pressure mercury acr lamp $(125 \mathrm{~W})$ for 6 hours. Solvent was evaporated and residual was purified by column chromatography (hexane/ethyl acetate 10:1). The obtained mixture of isomers 31 and 3la was separated by column chromatography (hexane/ethyl acetate 20:1). Pure 31 (170 mg, $0.559 \mathrm{mmol}, 6 \%$ yield) was obtained. ${ }^{1} \mathrm{H}$ NMR (400 MHz, Chloroform-d) $\delta$ $7.36(\mathrm{dd}, \mathrm{J}=1.8,0.8 \mathrm{~Hz}, 2 \mathrm{H}), 6.30(\mathrm{dd}, \mathrm{J}=3.2,1.9 \mathrm{~Hz}, 2 \mathrm{H}), 6.16(\mathrm{dd}, \mathrm{J}=3.2,0.8 \mathrm{~Hz}, 2 \mathrm{H})$, $3.77(\mathrm{~d}, \mathrm{~J}=9.6 \mathrm{~Hz}, 2 \mathrm{H}), 3.73(\mathrm{~s}, 6 \mathrm{H}), 3.54(\mathrm{~d}, \mathrm{~J}=9.6 \mathrm{~Hz}, 2 \mathrm{H}) .{ }^{13} \mathrm{C} \mathrm{NMR}(101 \mathrm{MHz}$, Chloroform-d) $\delta$ 167.46, 148.34, 137.47, 105.61, 101.92, 47.49, 38.27, 34.88. HRMS (ESI+) Calcd for $\mathrm{C}_{16} \mathrm{H}_{16} \mathrm{O}_{6}[\mathrm{M}+\mathrm{Na}]^{+}$327.08391, found: $327.08395 \mathrm{~m} / \mathrm{z}$. UV-VIS (acetonitrile): 223 $\mathrm{nm}$. 


\section{S3 $[2+2]$ cycloreversion reactions catalyzed by $1+\mathrm{TfOH}$}

\section{General procedure for cycloreversions on analytical scale}

Cyclobutane derivative $(0.02 \mathrm{mmol})$ and riboflavine tetraacetate (1, usually $0.001 \mathrm{mmol}, 2.5$ mol \%) were placed in Schlenk tube, anhydrous acetonitrile $(0.9 \mathrm{~mL})$ and $0.1 \mathrm{~mL}$ of $0.1 \mathrm{M}$ triflic acid in acetonitrile were added. Homogenous solution was three-times degassed using freeze-pump-thaw technique. The mixture was irradiated (LED, $400 \mathrm{~nm}$ ) for given time depending on cyclobutane substrate (usually $10 \mathrm{~min}$ ). The reaction mixture was analyzed by ${ }^{1} \mathrm{H}$ NMR.

\section{General procedure for cycloreversions on preparative scale}

Cyclobutane derivative $(0.4 \mathrm{mmol})$ and riboflavine tetraacetate $(1,0.02 \mathrm{mmol}, 5 \mathrm{~mol} \%)$ were placed in Schlenk tube, anhydrous acetonitrile $(19.8 \mathrm{~mL})$ and $0.2 \mathrm{~mL}$ of $1 \mathrm{M}$ triflic acid in acetonitrile were added. Homogenous solution was three-times degassed using freeze-pumpthaw technique. Mixture tube was irradiated (LED, $400 \mathrm{~nm}$ ) for given time depending on cyclobutane substrate. After irradiation, solvent was removed and crude product was purified by column chromatography to give pure product in good to high yields.

\section{1-Methylquinolin-2(1H)-one (4a)}

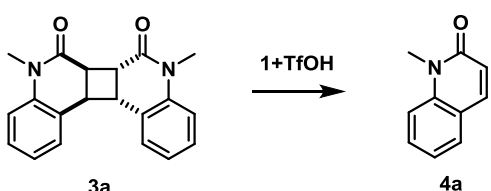

Solution containing 3a (127 mg, $0.4 \mathrm{mmol})$ and $\mathbf{1}(10.89 \mathrm{mg}, 0.020 \mathrm{mmol})$ was irradiated with LED $(400 \mathrm{~nm})$ for 10 minutes. Crude product was purified by column chromatography (dichloromethane) to afford $4 \mathbf{a}\left(118 \mathrm{mg}, 0.741 \mathrm{mmol}, 93 \%\right.$ yield). M.p. $72-75{ }^{\circ} \mathrm{C} .{ }^{1} \mathrm{H}$ NMR (400 MHz, Chloroform- $d$ ) $\delta 7.67(\mathrm{~d}, J=9.4 \mathrm{~Hz}, 1 \mathrm{H}), 7.61-7.53(\mathrm{~m}, 2 \mathrm{H}), 7.36(\mathrm{dd}, J=8.3$, $0.9 \mathrm{~Hz}, 1 \mathrm{H}), 7.29-7.18(\mathrm{~m}, 1 \mathrm{H}), 6.71(\mathrm{~d}, J=9.5 \mathrm{~Hz}, 1 \mathrm{H}), 3.72(\mathrm{~s}, 3 \mathrm{H}) .{ }^{13} \mathrm{C} \mathrm{NMR}(75 \mathrm{MHz}$, Chloroform- $d$ ) $\delta 162.52,140.22,139.15,130.83,128.94,122.29,121.92,120.86,114.34$, 29.60 .

2H-Chromen-2-one (4b) from $3 \boldsymbol{b}$

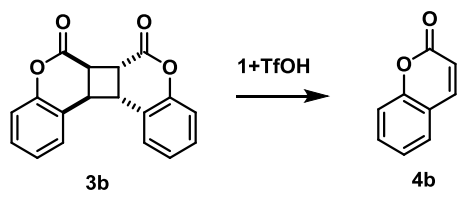


Solution containing 3b $(0.117 \mathrm{~g}, 0.400 \mathrm{mmol}), \mathbf{1}(10.90 \mathrm{mg}, 0.020 \mathrm{mmol})$ and trifluoromethanesulfonic acid $(0.200 \mathrm{~mL}, 0.200 \mathrm{mmol})$ was irradiated for 10 minutes using LED (400 nm). Crude product was purified by column chromatography (hexane/ethyl acetate 1:1) to afford 4b (105 mg, $0.718 \mathrm{mmol}, 90 \%$ yield). M.p. $69-71{ }^{\circ} \mathrm{C} .{ }^{1} \mathrm{H}$ NMR $(400 \mathrm{MHz}$, Chloroform- $d$ ) $\delta 7.71(\mathrm{dd}, J=9.6,0.6 \mathrm{~Hz}, 1 \mathrm{H}), 7.60-7.44(\mathrm{~m}, 2 \mathrm{H}), 7.39-7.26(\mathrm{~m}, 3 \mathrm{H}), 6.43$ $(\mathrm{d}, J=9.5 \mathrm{~Hz}, 1 \mathrm{H}) .{ }^{13} \mathrm{C}$ NMR (101 MHz, Chloroform-d) $\delta 160.76,154.05,143.39,131.82$, $127.83,124.40,118.82,116.92,116.72$.

2H-Chromen-2-one (4b) from $3 \boldsymbol{h}$

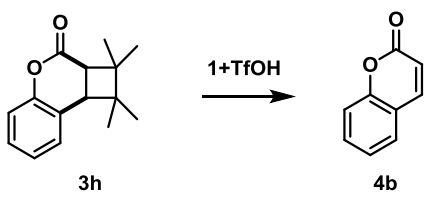

Solution containing $\mathbf{3 h}$ (92 $\mathrm{mg}, \quad 0.4 \mathrm{mmol}), \quad \mathbf{1}(10.90 \mathrm{mg}, 0.020 \mathrm{mmol})$ and trifluoromethanesulfonic acid $(0.200 \mathrm{~mL}, 0.200 \mathrm{mmol})$ was irradiated for 240 minutes using LED (400 nm). Crude product was purified by column chromatography (hexane/ethyl acetate 1:1) to afford $\mathbf{4 b}\left(40 \mathrm{mg}, 0.274 \mathrm{mmol}, 68 \%\right.$ yield). M.p. $69-71{ }^{\circ} \mathrm{C} .{ }^{1} \mathrm{H}$ NMR $(400 \mathrm{MHz}$, Chloroform- $d$ ) $\delta 7.71(\mathrm{dd}, J=9.6,0.6 \mathrm{~Hz}, 1 \mathrm{H}), 7.60-7.44(\mathrm{~m}, 2 \mathrm{H}), 7.39-7.26(\mathrm{~m}, 3 \mathrm{H}), 6.43$ $(\mathrm{d}, J=9.5 \mathrm{~Hz}, 1 \mathrm{H}) .{ }^{13} \mathrm{C} \mathrm{NMR}(101 \mathrm{MHz}$, Chloroform- $d) \delta 160.76,154.05,143.39,131.82$, $127.83,124.40,118.82,116.92,116.72$.

(Z)-3-(4-Methoxyphenyl) acrylic anhydride (4e)

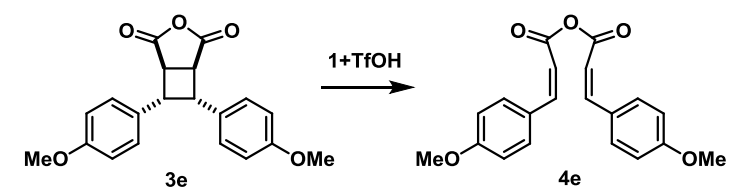

Solution containing $3 \mathrm{e}(0.135 \mathrm{~g}, \quad 0.4 \mathrm{mmol}), \quad \mathbf{1}(10.90 \mathrm{mg}, 0.020 \mathrm{mmol})$ and trifluoromethanesulfonic acid $(0.200 \mathrm{~mL}, 0.200 \mathrm{mmol})$ was irradiated for 30 minutes using LED (400 nm). Crude product was purified by column chromatography (hexane/ethyl acetate $10: 1)$ to afford $4 \mathrm{e}(0.111 \mathrm{~g}, 0.328 \mathrm{mmol}, 78 \%$ yield $)$. Polymerization of anhydride was observed. ${ }^{1} \mathrm{H}$ NMR (400 MHz, Chloroform- $d$ ) $\delta 7.26(\mathrm{~s}, 1 \mathrm{H}), 6.88-6.77$ (m, 4H), 6.66 (d, $J=$ $8.7 \mathrm{~Hz}, 4 \mathrm{H}), 4.35-4.28(\mathrm{~m}, 2 \mathrm{H}), 3.81(\mathrm{dd}, J=5.5,1.8 \mathrm{~Hz}, 2 \mathrm{H}), 3.70(\mathrm{~s}, 6 \mathrm{H}) .{ }^{13} \mathrm{C} \mathrm{NMR}(101$ MHz, Chloroform-d) $\delta 179.27,158.08,130.31,128.80,113.52,55.11,44.11,43.86$.

\section{1,3-Dimethylpyrimidine-2,4(1H,3H)-dione (4f)}

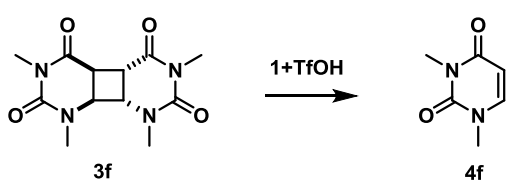


Solution containing $\mathbf{3 f}(0.115 \mathrm{~g}, \quad 0.410 \mathrm{mmol}), \quad \mathbf{1}(0.011 \mathrm{~g}, 0.021 \mathrm{mmol})$ and trifluoromethanesulfonic acid $(0.200 \mathrm{~mL}, 0.200 \mathrm{mmol})$ was irradiated for 10 minutes. Crude product was purified by column chromatography (dichloromethane/methanol 10:1) to afford 4f (0.108 g, $0.771 \mathrm{mmol}, 94 \%$ yield). M.p. $118-119{ }^{\circ} \mathrm{C} .{ }^{1} \mathrm{H}$ NMR (400 MHz, Chloroform- $\left.d\right) \delta$ $7.12(\mathrm{~d}, J=7.9 \mathrm{~Hz}, 1 \mathrm{H}), 5.73(\mathrm{~d}, J=7.8 \mathrm{~Hz}, 1 \mathrm{H}), 3.39$ (s, 3H), 3.34 (s, 3H). ${ }^{13} \mathrm{C}$ NMR $(101$ MHz, Chloroform- $d$ ) $\delta 163.35,151.92,142.66,101.38,37.02,27.75$.

\section{1,3-Dimethylpyrimidine-2,4(1H,3H)-dione (4f)}

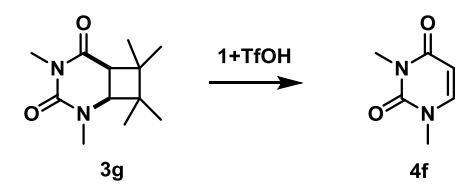

Solution containing $\mathbf{3 g}$ (90 $\mathrm{mg}, 0.401 \mathrm{mmol}), \quad 1 \quad(10.92 \mathrm{mg}, 0.020 \mathrm{mmol})$ and trifluoromethanesulfonic acid $(0.200 \mathrm{~mL}, 0.200 \mathrm{mmol})$ was irradiated for 120 minutes. Crude product was purified by column chromatography (dichloromethane/methanol 10:1) to afford 4f (35 mg, $0.250 \mathrm{mmol}, 62 \%$ yield). M.p. $118-119^{\circ} \mathrm{C} .{ }^{1} \mathrm{H}$ NMR (400 MHz, Chloroform- $d$ ) $\delta$ $7.12(\mathrm{~d}, J=7.9 \mathrm{~Hz}, 1 \mathrm{H}), 5.73(\mathrm{~d}, J=7.8 \mathrm{~Hz}, 1 \mathrm{H}), 3.39(\mathrm{~s}, 3 \mathrm{H}), 3.34(\mathrm{~s}, 3 \mathrm{H}) .{ }^{13} \mathrm{C}$ NMR $(101$ $\mathrm{MHz}$, Chloroform-d) $\delta 163.35,151.92,142.66,101.38,37.02,27.75$.

Methyl (E)-3-(4-methoxyphenyl)acrylate (4h)

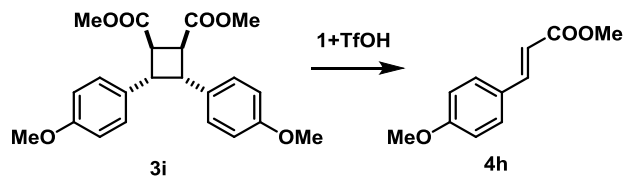

Solution containing $\mathbf{3 i}(0.154 \mathrm{~g}, \quad 0.4 \mathrm{mmol}), \quad 1 \quad(10.90 \mathrm{mg}, \quad 0.020 \mathrm{mmol})$ and trifluoromethanesulfonic acid $(0.200 \mathrm{~mL}, 0.200 \mathrm{mmol})$ was irradiated for 30 minutes. Crude product was purified by column chromatography (hexane/ethyl acetate 10:1) to afford $\mathbf{4 h}$ (0.066 g, 0.344 mmol, 86\% yield). M.p. 89-92 ${ }^{\circ} \mathrm{C} .{ }^{1} \mathrm{H}$ NMR (400 MHz, Chloroform- $d$ ) $\delta 7.65$ $(\mathrm{d}, J=16.0 \mathrm{~Hz}, 1 \mathrm{H}), 7.52-7.40(\mathrm{~m}, 2 \mathrm{H}), 6.90(\mathrm{~d}, J=8.8 \mathrm{~Hz}, 2 \mathrm{H}), 6.31(\mathrm{~d}, J=16.0 \mathrm{~Hz}, 2 \mathrm{H})$, $3.83(\mathrm{~s}, 3 \mathrm{H}), 3.79(\mathrm{~s}, 3 \mathrm{H}) .{ }^{13} \mathrm{C}$ NMR (101 MHz, Chloroform-d) $\delta$ 167.75, 161.36, 144.51, $129.70,127.09,115.24,114.30,55.36,51.57$.

(E)-1,2-Bis(4-methoxyphenyl)ethene (4i)

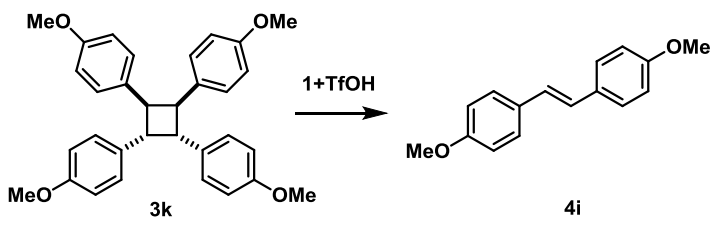


Solution containing $\mathbf{3 k}(0.192 \mathrm{~g}, \quad 0.4 \mathrm{mmol}), \quad \mathbf{1}(10.90 \mathrm{mg}, 0.020 \mathrm{mmol})$ and trifluoromethanesulfonic acid $(0.200 \mathrm{~mL}, 0.200 \mathrm{mmol})$ was irradiated for 30 minutes. Crude product was purified by column chromatography (hexane/ethyl acetate 10:1) to afford $\mathbf{4 i}$ (0.086 g, $0.356 \mathrm{mmol}, 89 \%$ yield) containing $10 \%$ of Z-isomer. M.p. $211-214{ }^{\circ} \mathrm{C} .{ }^{1} \mathrm{H}$ NMR (400 MHz, Chloroform-d) $\delta 7.43$ (d, $J=8.7 \mathrm{~Hz}, 4 \mathrm{H}), 6.93$ (s, 2H), 6.89 (d, $J=8.7 \mathrm{~Hz}, 4 \mathrm{H})$, 3.83 (s, 6H). ${ }^{13} \mathrm{C}$ NMR (101 MHz, Chloroform- $d$ ) $\delta$ 158.96, 130.44, 127.39, 126.14, 114.07, 55.32. Z-isomer: ${ }^{1} \mathrm{H}$ NMR (400 MHz, Chloroform-d) $\delta 7.22(\mathrm{~d}, \mathrm{~J}=7.8 \mathrm{~Hz}, 4 \mathrm{H}), 6.78(\mathrm{~d}, \mathrm{~J}=$ $7.8 \mathrm{~Hz}, 4 \mathrm{H}), 6.46$ (s, 2H), 3.80 (s, 6H). ${ }^{13} \mathrm{C}$ NMR (75 MHz, Chloroform-d) $\delta 158.4,130.0$, $129.9,128.3,113.5,55.2$. 


\section{General procedure for cycloreversions on analytical scale}

Cyclobutane derivative $(0.02 \mathrm{mmol})$ and 5-ethyl-1,3-dimethyl-8-trifluoromethylalloxazinium perchlorate (2c; $0.001 \mathrm{mmol}, 2.5 \mathrm{~mol} \%)$ were placed in Schlenk tube and anhydrous acetonitrile $(1 \mathrm{~mL})$ was added. Homogenous solution was three-times deggased using freezepump-thaw technique. Solution in Schlenk tube was irradiated (LED, $450 \mathrm{~nm}$ ) for given time depending on cyclobutane substrate (usually $10 \mathrm{~min}$ ). The reaction mixture was analyzed by ${ }^{1} \mathrm{H}$ NMR.

\section{General procedure for cycloreversions on preparative scale}

Cyclobutane derivative $(0.4 \mathrm{mmol})$ and 5-ethyl-1,3-dimethyl-8-trifluoromethylalloxazinium perchlorate (2c; $0.02 \mathrm{mmol}, 5 \mathrm{~mol} \%)$ were placed in Schlenk tube and anhydrous acetonitrile $(20 \mathrm{~mL})$ was added. Homogenous solution was three-times deggased using freeze-pump-thaw technique. Solution in Schlenk tube was irradiated (LED, $450 \mathrm{~nm}$ ) for given time depending on cyclobutane substrate. After irradiation, solvent was removed and crude product purified by column chromatography to give pure product in good to high yield.

\section{1-Methylquinolin-2(1H)-one (4a)}

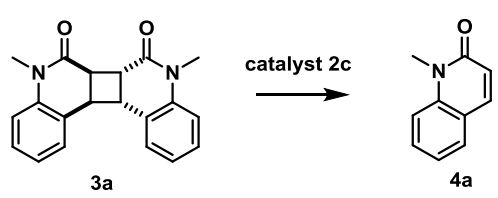

Solution containing $\mathbf{2 c}(8.77 \mathrm{mg}, 0.020 \mathrm{mmol})$ and $\mathbf{3 a}(0.127 \mathrm{~g}, 0.4 \mathrm{mmol})$ in acetonitrile (20 $\mathrm{mL}$ ) was irradiated for 30 minutes using LED $(450 \mathrm{~nm})$. Crude product was purified by column cromatography (dihloromethane/methanol 20:1) to afford $4 \mathbf{a}(0.056 \mathrm{~g}, 0.352 \mathrm{mmol}$, 88\% yield). M.p. $72-75{ }^{\circ} \mathrm{C} .{ }^{1} \mathrm{H}$ NMR (400 MHz, Chloroform-d) $\delta 7.67$ (d, $\left.J=9.4 \mathrm{~Hz}, 1 \mathrm{H}\right)$, $7.61-7.53(\mathrm{~m}, 2 \mathrm{H}), 7.36(\mathrm{dd}, J=8.3,0.9 \mathrm{~Hz}, 1 \mathrm{H}), 7.29-7.18(\mathrm{~m}, 1 \mathrm{H}), 6.71(\mathrm{~d}, J=9.5 \mathrm{~Hz}$, 1H), $3.72(\mathrm{~s}, 3 \mathrm{H}) .{ }^{13} \mathrm{C}$ NMR (75 MHz, Chloroform- $d$ ) $\delta 162.52,140.22,139.15,130.83$, $128.94,122.29,121.92,120.86,114.34,29.60$.

2H-Chromen-2-one (4b) from $3 \boldsymbol{b}$

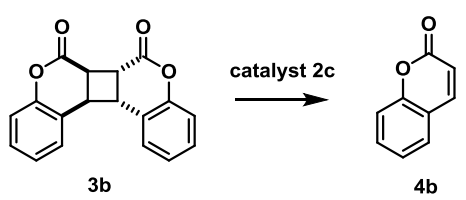

Solution containing $2 \mathbf{c}(8.77 \mathrm{mg}, 0.020 \mathrm{mmol})$ and $\mathbf{3 b}(0.117 \mathrm{~g}, 0.4 \mathrm{mmol})$ in acetonitrile (20 $\mathrm{mL}$ ) was irradiated for 30 minutes using LED $(450 \mathrm{~nm})$. Crude product was purified by 
column chromatography (hexane/ethyl acetate 1:1) to afford $\mathbf{4 b}(0.108 \mathrm{~g}, 0.736 \mathrm{mmol}, 92 \%$ yield). M.p. $69-71{ }^{\circ} \mathrm{C} .{ }^{1} \mathrm{H}$ NMR (400 MHz, Chloroform- $d$ ) $\delta 7.71(\mathrm{dd}, J=9.6,0.6 \mathrm{~Hz}, 1 \mathrm{H})$, $7.60-7.44(\mathrm{~m}, 2 \mathrm{H}), 7.39-7.26(\mathrm{~m}, 3 \mathrm{H}), 6.43(\mathrm{~d}, J=9.5 \mathrm{~Hz}, 1 \mathrm{H}) .{ }^{13} \mathrm{C}$ NMR $(101 \mathrm{MHz}$, Chloroform- $d$ ) $\delta 160.76,154.05,143.39,131.82,127.83,124.40,118.82,116.92,116.72$.

2H-Chromen-2-one (4b) from $3 \boldsymbol{h}$

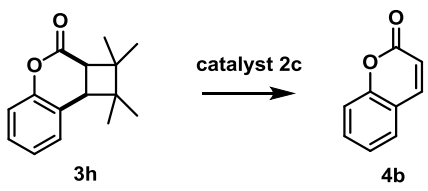

Solution containing $\mathbf{2 c}(8.77 \mathrm{mg}, 0.020 \mathrm{mmol})$ and $\mathbf{3 h}(0.092 \mathrm{~g}, 0.4 \mathrm{mmol})$ in acetonitrile (20 $\mathrm{mL}$ ) was irradiated for 30 minutes using LED $(450 \mathrm{~nm})$. Crude product was purified by column chromatography (hexane/ethyl acetate $1: 1)$ to afford $\mathbf{4 b}(0.029 \mathrm{~g}, 0.200 \mathrm{mmol}, 50 \%$ yield). M.p. $69-71{ }^{\circ} \mathrm{C} .{ }^{1} \mathrm{H}$ NMR (400 MHz, Chloroform- $d$ ) $\delta 7.71$ (dd, $\left.J=9.6,0.6 \mathrm{~Hz}, 1 \mathrm{H}\right)$, $7.60-7.44(\mathrm{~m}, 2 \mathrm{H}), 7.39-7.26(\mathrm{~m}, 3 \mathrm{H}), 6.43(\mathrm{~d}, J=9.5 \mathrm{~Hz}, 1 \mathrm{H}) .{ }^{13} \mathrm{C}$ NMR $(101 \mathrm{MHz}$, Chloroform- $d$ ) $\delta 160.76,154.05,143.39,131.82,127.83,124.40,118.82,116.92,116.72$.

\section{1,1-Dimethylnaphthalen-2(1H)-one (4c)}

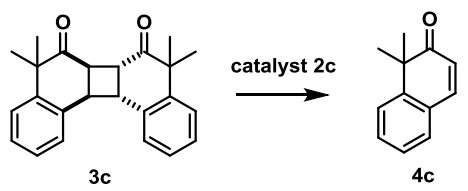

Solution containing $2 \mathbf{c}(8.77 \mathrm{mg}, 0.020 \mathrm{mmol})$ and $3 \mathbf{c}(0.138 \mathrm{~g}, 0.4 \mathrm{mmol})$ in nitromethane $(20 \mathrm{~mL})$ was irradiated for 240 minutes using LED $(450 \mathrm{~nm})$. Crude product was purified by column chromatography (hexane/ethylacetate $10: 1)$ to afford $\mathbf{4 c}(0.072 \mathrm{~g}, 0.416 \mathrm{mmol}, 52 \%$ yield). M.p. $71-74{ }^{\circ} \mathrm{C} .{ }^{1} \mathrm{H}$ NMR (400 MHz, Chloroform- $d$ ) $\delta 7.50-7.28$ (m, 8H), $4.19-4.09$ $(\mathrm{m}, 2 \mathrm{H}), 3.81(\mathrm{dt}, J=8.4,1.4 \mathrm{~Hz}, 2 \mathrm{H}), 1.58(\mathrm{~s}, 6 \mathrm{H}), 1.38(\mathrm{~s}, 6 \mathrm{H}) .{ }^{13} \mathrm{C}$ NMR $(101 \mathrm{MHz}$, Chloroform- $d$ ) $\delta 208.94,139.99,132.63,123.97,123.47,123.30,121.50,44.63,43.54,38.14$, 25.04, 17.23.

(Z)-3-(4-Methoxyphenyl)acrylic anhydride (4e)

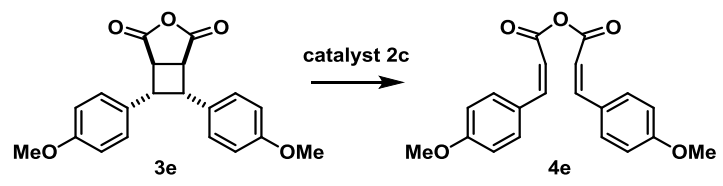

Solution containing $3 \mathbf{e}(0.135 \mathrm{~g}, 0.4 \mathrm{mmol})$ and $\mathbf{2 c}(8.77 \mathrm{mg}, 0.020 \mathrm{mmol})$ in acetonitrile (20 $\mathrm{mL}$ ) was irradiated for 30 minutes using LED $(450 \mathrm{~nm})$. Crude product was purified by column chromatography (hexane/ethyl acetate $10: 1)$ to afford $4 \mathbf{e}(0.111 \mathrm{~g}, 0.328 \mathrm{mmol}, 86 \%$ 
yield). ${ }^{1} \mathrm{H}$ NMR (400 MHz, Chloroform- $d$ ) $\delta 7.26(\mathrm{~s}, 1 \mathrm{H}), 6.88-6.77$ (m, 4H), 6.66 (d, $J=$ $8.7 \mathrm{~Hz}, 4 \mathrm{H}), 4.35-4.28(\mathrm{~m}, 2 \mathrm{H}), 3.81(\mathrm{dd}, J=5.5,1.8 \mathrm{~Hz}, 2 \mathrm{H}), 3.70(\mathrm{~s}, 6 \mathrm{H}) .{ }^{13} \mathrm{C} \mathrm{NMR}(101$ $\mathrm{MHz}$, Chloroform- $d$ ) $\delta$ 179.27, 158.08, 130.31, 128.80, 113.52, 55.11, 44.11, 43.86.

\section{1,3-Dimethylpyrimidine-2,4(1H,3H)-dione (4f) from $\mathbf{3 f}$}

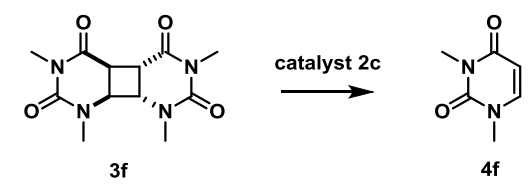

Solution containing $\mathbf{3 f}(112 \mathrm{mg}, 0.4 \mathrm{mmol})$ and $2 \mathrm{c}(8.77 \mathrm{mg}, 0.020 \mathrm{mmol})$ in acetonitrile (20 $\mathrm{mL}$ ) was irradiated for 30 minutes using LED $(450 \mathrm{~nm})$. Crude product was purified by column chromatography (dichloromethane/methanol 10:1) to afford $\mathbf{4 f}$ (102 $\mathrm{mg}, 0.728 \mathrm{mmol}$, 91\% yield). M.p. $118-119{ }^{\circ} \mathrm{C} .{ }^{1} \mathrm{H}$ NMR (400 MHz, Chloroform- $d$ ) $\delta 7.12$ (d, $\left.J=7.9 \mathrm{~Hz}, 1 \mathrm{H}\right)$, $5.73(\mathrm{~d}, J=7.8 \mathrm{~Hz}, 1 \mathrm{H}), 3.39(\mathrm{~s}, 3 \mathrm{H}), 3.34(\mathrm{~s}, 3 \mathrm{H}) .{ }^{13} \mathrm{C}$ NMR $(101 \mathrm{MHz}$, Chloroform- $d) \delta$ $163.35,151.92,142.66,101.38,37.02,27.75$.

\section{1,3-Dimethylpyrimidine-2,4(1H,3H)-dione (4f) from $3 \mathbf{g}$}

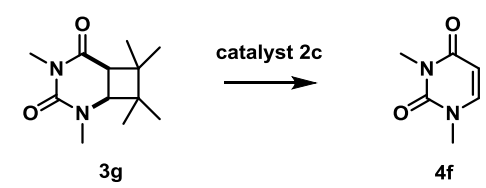

Solution containing $\mathbf{3 g}(0.090 \mathrm{~g}, 0.4 \mathrm{mmol})$ and $2 \mathbf{c}(8.77 \mathrm{mg}, 0.020 \mathrm{mmol})$ in acetonitrile (20 $\mathrm{mL}$ was irradiated for 120 minutes using LED $(450 \mathrm{~nm})$. Crude product was purified by column chromatography (dichloromethane/methanol 10:1) to affrod $\mathbf{4 f}(0.042 \mathrm{~g}, 0.300 \mathrm{mmol}$, 75\% yield). M.p. $118-119{ }^{\circ} \mathrm{C} .{ }^{1} \mathrm{H}$ NMR (400 MHz, Chloroform- $d$ ) $\delta 7.12$ (d, $\left.J=7.9 \mathrm{~Hz}, 1 \mathrm{H}\right)$, $5.73(\mathrm{~d}, J=7.8 \mathrm{~Hz}, 1 \mathrm{H}), 3.39$ (s, 3H), 3.34 (s, 3H). ${ }^{13} \mathrm{C}$ NMR (101 MHz, Chloroform- $d$ ) $\delta$ $163.35,151.92,142.66,101.38,37.02,27.75$.

Methyl (E)-3-(4-methoxyphenyl)acrylate (4h)

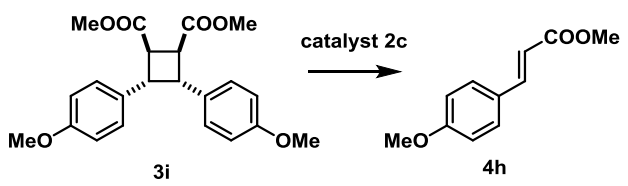

Solution containing $2 \mathbf{c}(8.77 \mathrm{mg}, 0.020 \mathrm{mmol})$ and $\mathbf{3 i}(0.154 \mathrm{~g}, 0.4 \mathrm{mmol})$ in acetonitrile (20 $\mathrm{mL}$ was irradiated for 30 minutes using LED $(450 \mathrm{~nm})$. Crude product was purified by column chromatography (hexane/ethyl acetate $10: 1)$ to afford $4 \mathrm{~h}(0.145 \mathrm{~g}, 0.752 \mathrm{mmol}, 94 \%$ yield). M.p. 89-92 ${ }^{\circ} \mathrm{C} .{ }^{1} \mathrm{H}$ NMR (400 MHz, Chloroform- $d$ ) $\delta 7.65$ (d, $\left.J=16.0 \mathrm{~Hz}, 1 \mathrm{H}\right), 7.52$ 7.40 (m, 2H), 6.90 (d, $J=8.8 \mathrm{~Hz}, 2 \mathrm{H}), 6.31$ (d, $J=16.0 \mathrm{~Hz}, 2 \mathrm{H}), 3.83$ (s, 3H), 3.79 (s, 3H). 
${ }^{13} \mathrm{C}$ NMR (101 MHz, Chloroform-d) $\delta$ 167.75, 161.36, 144.51, 129.70, 127.09, 115.24, $114.30,55.36,51.57$.

(E)-1,2-Bis(4-methoxyphenyl)ethene (4i)

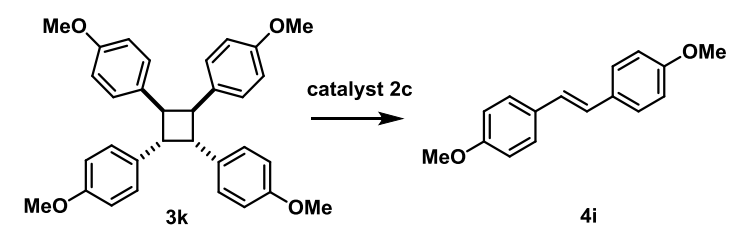

Solution containing 3k (192 mg, $0.4 \mathrm{mmol})$ and $\mathbf{2 c}(8.77 \mathrm{mg}, 0.020 \mathrm{mmol})$ in acetonitrile (20 $\mathrm{mL}$ ) was irradiated for 30 minutes using LED $(450 \mathrm{~nm})$. Crude product chromatographed using hexane/ethylacetate 10:1 to give pure (E)-1,2-bis(4-methoxyphenyl)ethene 4i (143mg, $0.595 \mathrm{mmol}, 88 \%$ yield) containing $10 \%$ of Z-isomer. M.p. $211-214{ }^{\circ} \mathrm{C} .{ }^{1} \mathrm{H}$ NMR $(400 \mathrm{MHz}$, Chloroform- $d$ ) $\delta 7.43(\mathrm{~d}, J=8.7 \mathrm{~Hz}, 4 \mathrm{H}), 6.93(\mathrm{~s}, 2 \mathrm{H}), 6.89$ (d, $J=8.7 \mathrm{~Hz}, 4 \mathrm{H}), 3.83(\mathrm{~s}, 6 \mathrm{H})$. ${ }^{13} \mathrm{C}$ NMR (101 MHz, Chloroform- $d$ ) $\delta$ 158.96, 130.44, 127.39, 126.14, 114.07, 55.32. Zisomer: ${ }^{1} \mathrm{H}$ NMR (400 MHz, Chloroform-d) $\delta 7.22(\mathrm{~d}, \mathrm{~J}=7.8 \mathrm{~Hz}, 4 \mathrm{H}), 6.78(\mathrm{~d}, \mathrm{~J}=7.8 \mathrm{~Hz}$, 4H), 6.46 (s, 2H), $3.80(\mathrm{~s}, 6 \mathrm{H}) .{ }^{13} \mathrm{C}$ NMR (75 MHz, Chloroform-d) $\delta$ 158.4, 130.0, 129.9, $128.3,113.5,55.2$.

Methyl (E)-3-(furan-2-yl)acrylate (4j)

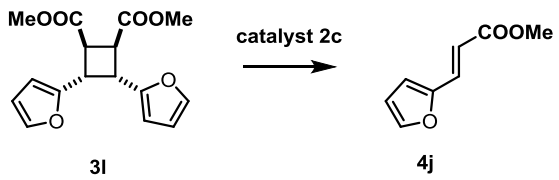

Solution containing $2 \mathrm{c}(8.77 \mathrm{mg}, 0.020 \mathrm{mmol})$ and $\mathbf{3 l}(0.122 \mathrm{~g}, 0.4 \mathrm{mmol})$ in acetonitrile (20 $\mathrm{mL}$ ) was irradiated for 30 minutes using LED $(450 \mathrm{~nm})$. Crude product was purified by column chromatography (hexane/ethyl acetate 10:1) to afford $4 \mathbf{j}(0.105 \mathrm{~g}, 0.688 \mathrm{mmol}, 86 \%$ yield). ${ }^{1} \mathrm{H}$ NMR (400 MHz, Chloroform-d) $\delta 7.48$ (dt, J = 1.8, $\left.0.6 \mathrm{~Hz}, 1 \mathrm{H}\right), 7.43$ (d, J = 15.7 $\mathrm{Hz}, 1 \mathrm{H}), 6.61(\mathrm{dd}, \mathrm{J}=3.4,0.6 \mathrm{~Hz}, 1 \mathrm{H}), 6.46(\mathrm{dd}, \mathrm{J}=3.4,1.8 \mathrm{~Hz}, 1 \mathrm{H}), 6.31(\mathrm{~d}, \mathrm{~J}=15.7 \mathrm{~Hz}$, 1H), 3.78 (s, 3H). ${ }^{13} \mathrm{C}$ NMR (101 MHz, Chloroform-d) $\delta 167.46,150.85,144.71,131.17$, 115.43, 114.76, 112.24, 51.64. 


\section{S5 Solvent screening in cycloreversion reactions}

Solvent screening was done under conditions of experiments on analytical scale, see S2 and $\mathrm{S} 3$ for General procedure.

\begin{tabular}{|c|c|}
\hline Solvent & Conversion $(10 \mathrm{~min})$ \\
\hline Acetonitrile & 100 \\
\hline $\mathrm{CHCl}_{3}$ & 8 \\
\hline DMF & 0 \\
\hline Dioxane & 0 \\
\hline Acetone & 24 \\
\hline Toluene & 0 \\
\hline $\mathrm{MeOH}$ & 0 \\
\hline $\mathrm{MeNO}_{2}$ & 100 \\
\hline
\end{tabular}

Dichloromethane

40

Reaction conditions: $3 \mathbf{b}(0 . \overline{\mathrm{mmol}})$, photocatalyst (2.5 mol\%), TfOH $(0.01 \mathrm{mmol})$, solvent $(1 \mathrm{~mL})$, visible light, rt, Ar.

\begin{tabular}{cc} 
Colvent & Conversion $(10 \mathrm{~min})$ \\
\hline Acetonitrile & 100 \\
$\mathrm{CHCl}_{3}$ & 6 \\
$\mathrm{DMF}$ & 11 \\
Dioxane & 0 \\
Acetone & 12 \\
Toluene & 6 \\
MeOH & 3 \\
MeNO & 85 \\
Dichloromethane & 5
\end{tabular}

Reaction conditions: $3 \mathbf{b}(0.02 \mathrm{mmol})$, photocatalyst $(2.5 \mathrm{~mol} \%)$, solvent $(1 \mathrm{~mL})$, visible light, $\mathrm{rt}$, Ar. 
UV-VIS spectrum of $\mathbf{1}$ in acetonitrile $\left(c(\mathbf{1})=1 \times 10^{-5} \mathrm{M}\right)$

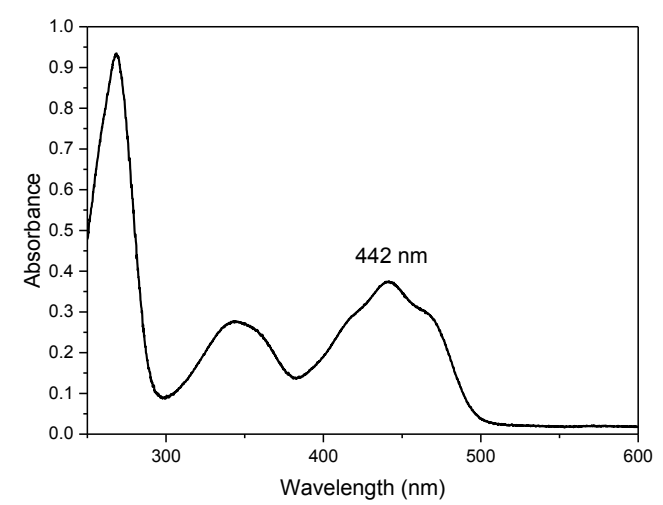

Fluorescence spectrum of $\mathbf{1}$ in acetonitrile $\left(c(\mathbf{1})=1 \times 10^{-6} \mathrm{M}\right)$

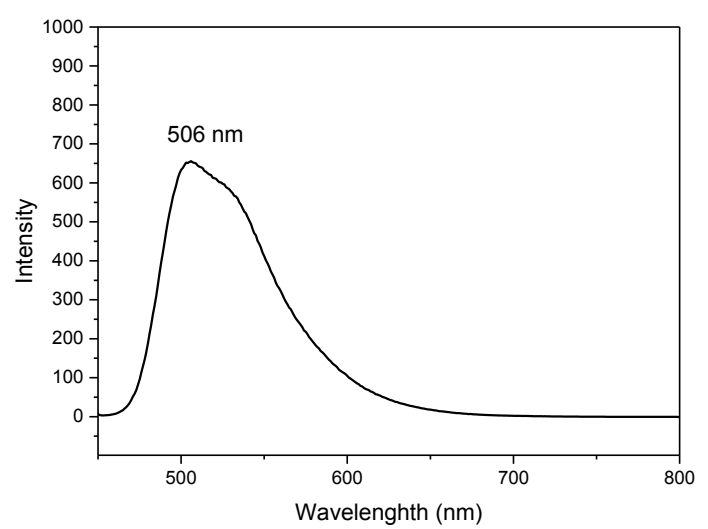

UV-VIS spectrum of $\mathbf{1}+\mathbf{T f O H}$ in acetonitrile $\left(c(\mathbf{1})=1 \times 10^{-5} \mathrm{M} ; c(\mathrm{TfOH})=0.01 \mathrm{M}\right)$

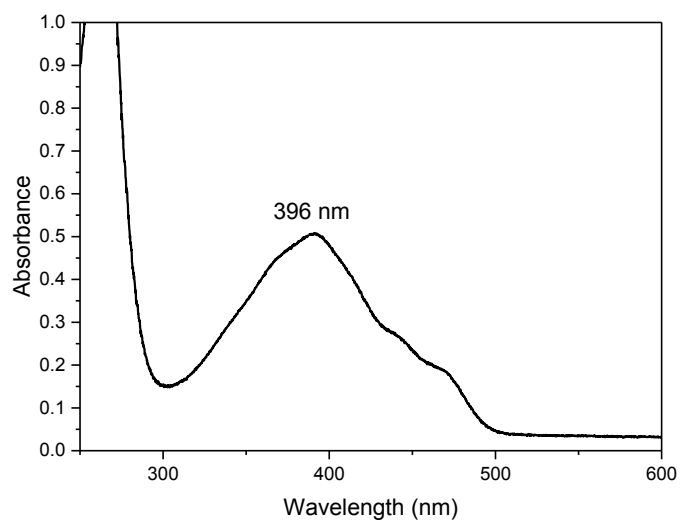


Fluorescence spectrum of $\mathbf{1}+\mathbf{T f O H}$ in acetonitrile $\left(c(\mathbf{1})=1 \times 10^{-5} \mathrm{M} ; c(\mathrm{TfOH})=0.01 \mathrm{M}\right)$

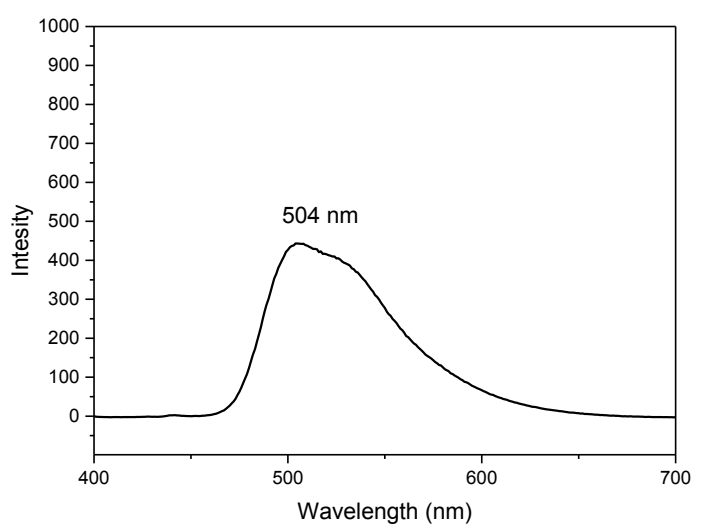

UV-VIS spectrum of $\mathbf{5}+\mathbf{T f O H}$ in acetonitrile $\left(c(\mathbf{5})=1 \times 10^{-5} \mathrm{M} ; c(\mathrm{TfOH})=0.01 \mathrm{M}\right)$

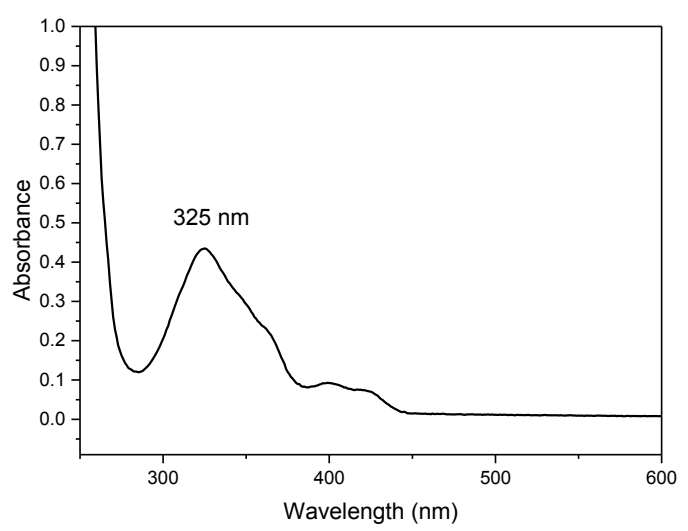

Fluorescence spectrum of $\mathbf{5}+\mathbf{T f O H}$ in acetonitrile $\left(c(\mathbf{5})=1 \times 10^{-5} \mathrm{M} ; c(\mathrm{TfOH})=0.01 \mathrm{M}\right)$

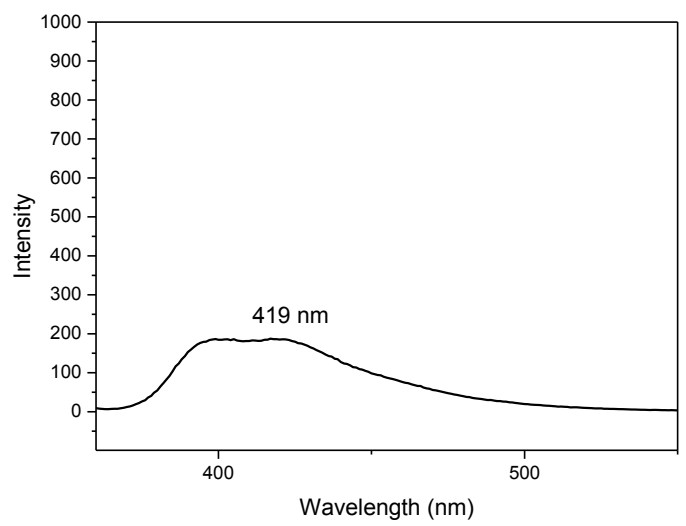


UV-VIS spectrum of $\mathbf{2 a}$ in acetonitrile $\left(c(\mathbf{2 a})=3.75 \times 10^{-5} \mathrm{M}\right)$

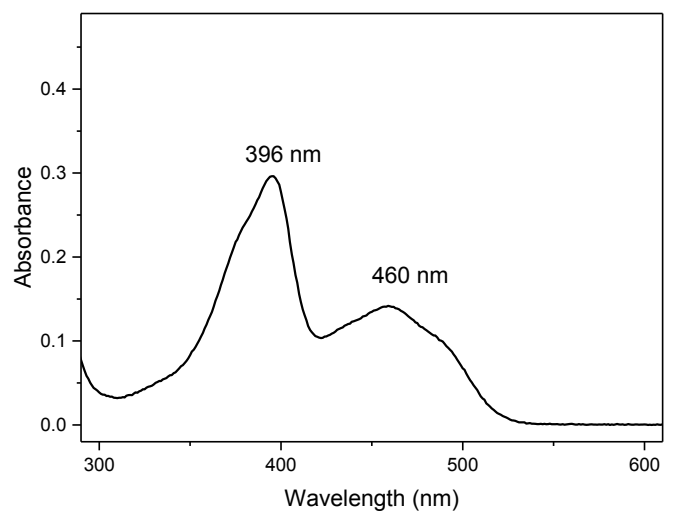

Fluorescence spectrum of $\mathbf{2 a}$ in acetonitrile $\left(c(\mathbf{2 a})=2.5 \times 10^{-5} \mathrm{M}\right)$

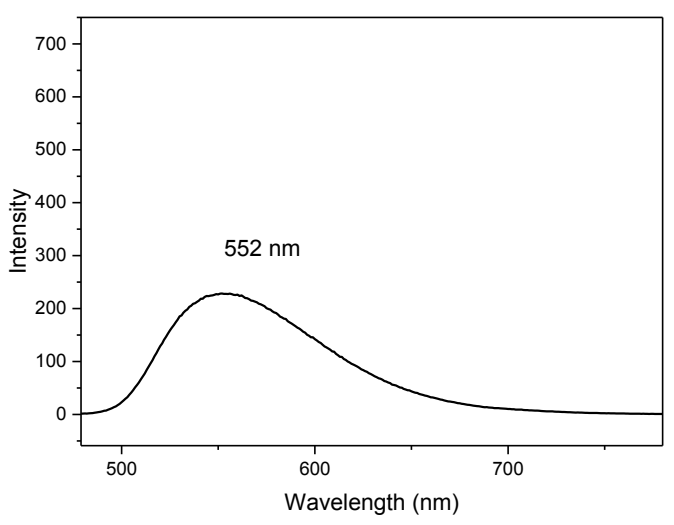

UV-VIS spectrum of $\mathbf{2 b}$ in acetonitrile $\left(c(\mathbf{2} \mathbf{b})=3.75 \times 10^{-5} \mathrm{M}\right)$

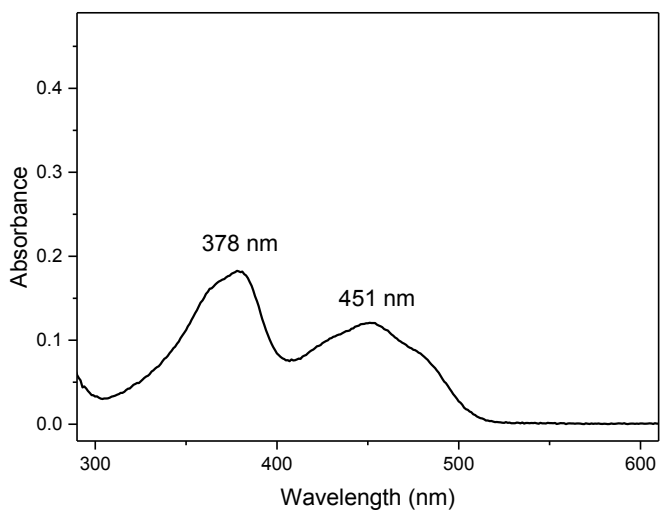


Fluorescence spectrum of $\mathbf{2 b}$ in acetonitrile $\left(c(\mathbf{2 b})=2.5 \times 10^{-5} \mathrm{M}\right)$

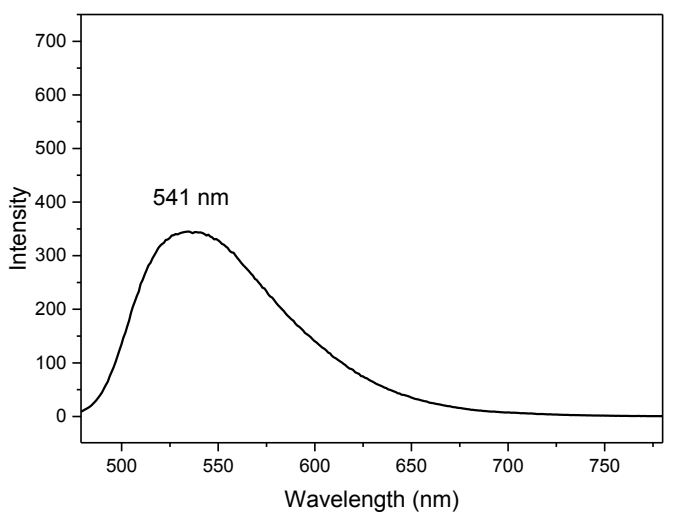

UV-VIS spectrum of $\mathbf{2 c}$ in acetonitrile $\left(c(\mathbf{2 c})=3.75 \times 10^{-5} \mathrm{M}\right)$

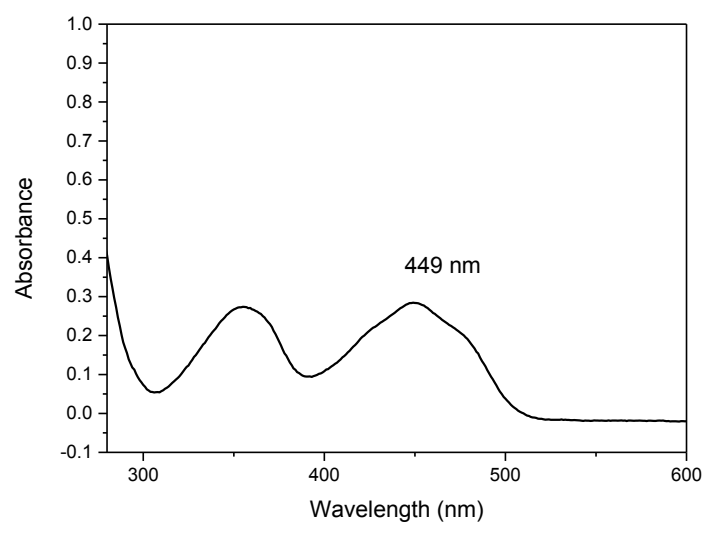

Fluorescence spectrum of $2 \mathrm{c}$ in acetonitrile $\left(c(\mathbf{1})=2.5 \times 10^{-5} \mathrm{M}\right)$

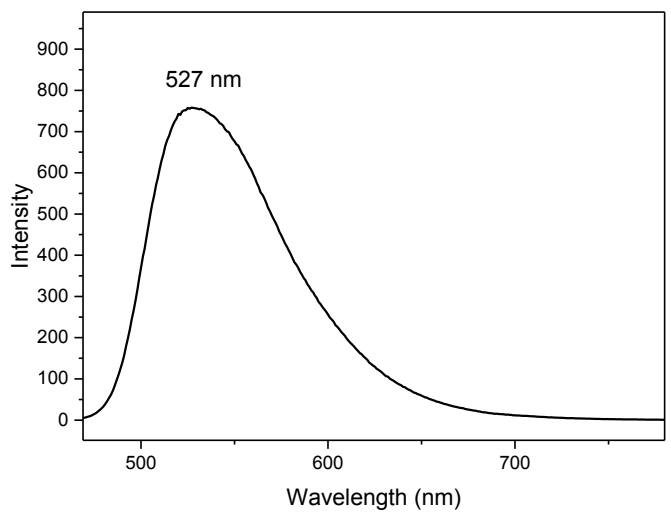


S7 Fluorescence quenching of catalysts 1 and 2 with selected cyclobutanes $3 \mathrm{a}, 3 \mathrm{~b}$

Stern-Volmer constants $K_{\mathrm{S}}$ for the electron transfer from $\mathbf{3 a}$ or $\mathbf{3 b}$ to flavin $\mathbf{1}$ (in the presence of $\mathrm{TfOH}, c(\mathrm{TfOH}=0.01 \mathrm{M})$ and $\mathbf{2}$ in acetonitrile.

\begin{tabular}{ccc}
\hline catalyst & Quencher & $K \mathrm{~s}\left(\mathrm{~L} \mathrm{~mol}^{-1}\right)$ \\
\hline 1+TfOH & 3b & 62 \\
2a & 3b & 8 \\
2b & 3b & 16 \\
2c & 3b & 29 \\
2c & 3a & 65 \\
\hline
\end{tabular}

Quenching of 1 with $\mathbf{3 b}$

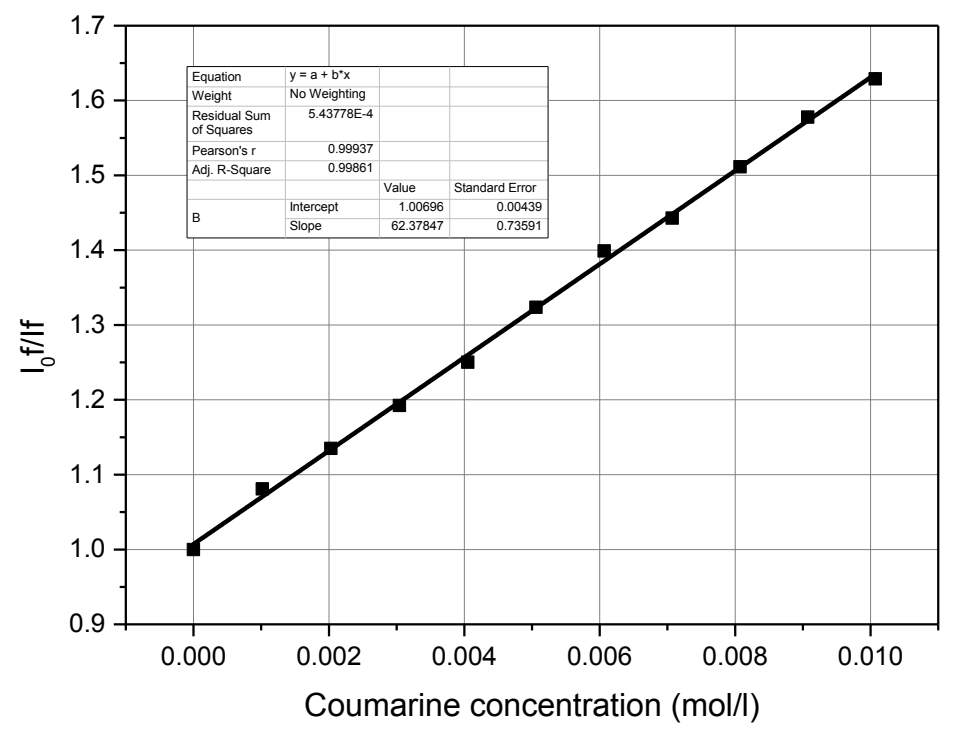


Quenching of 2a with 3b

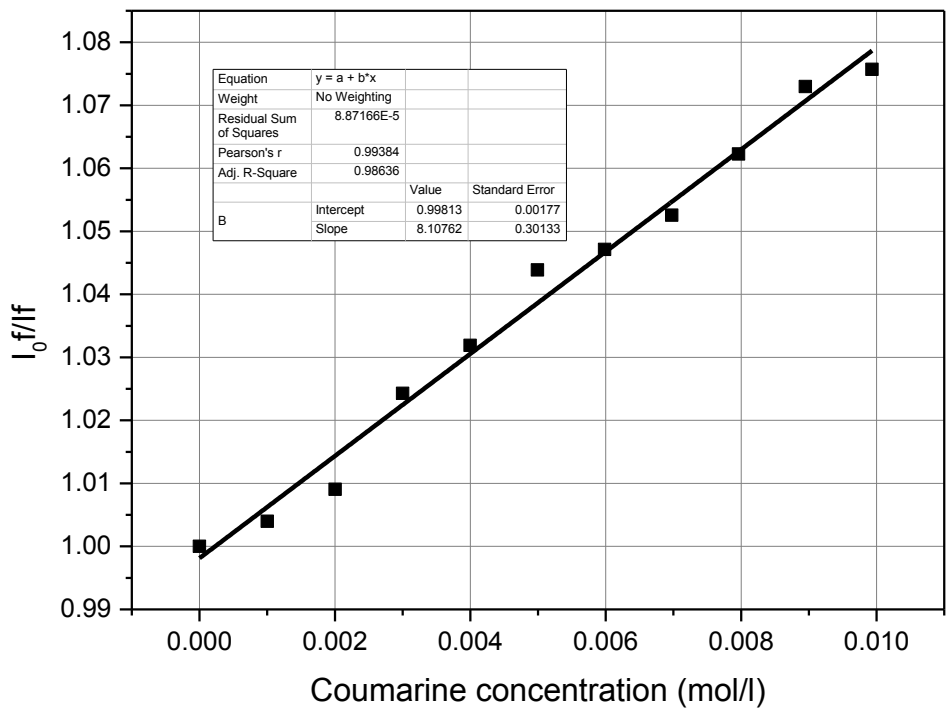

Quenching of $\mathbf{2 b}$ with $\mathbf{3 b}$

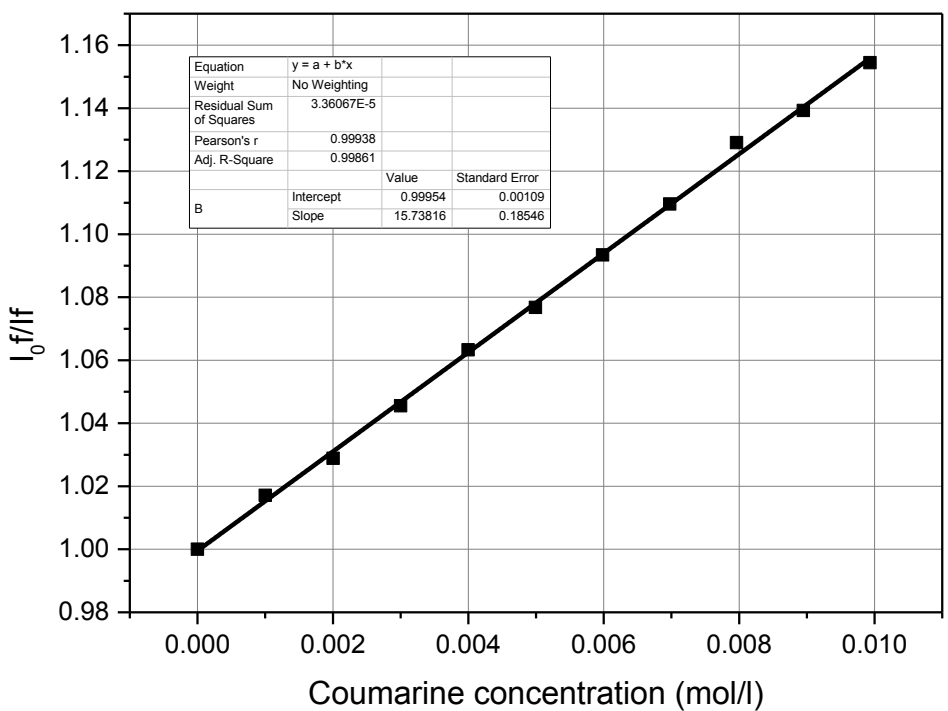


Quenching of $\mathbf{2 c}$ with $\mathbf{3 b}$

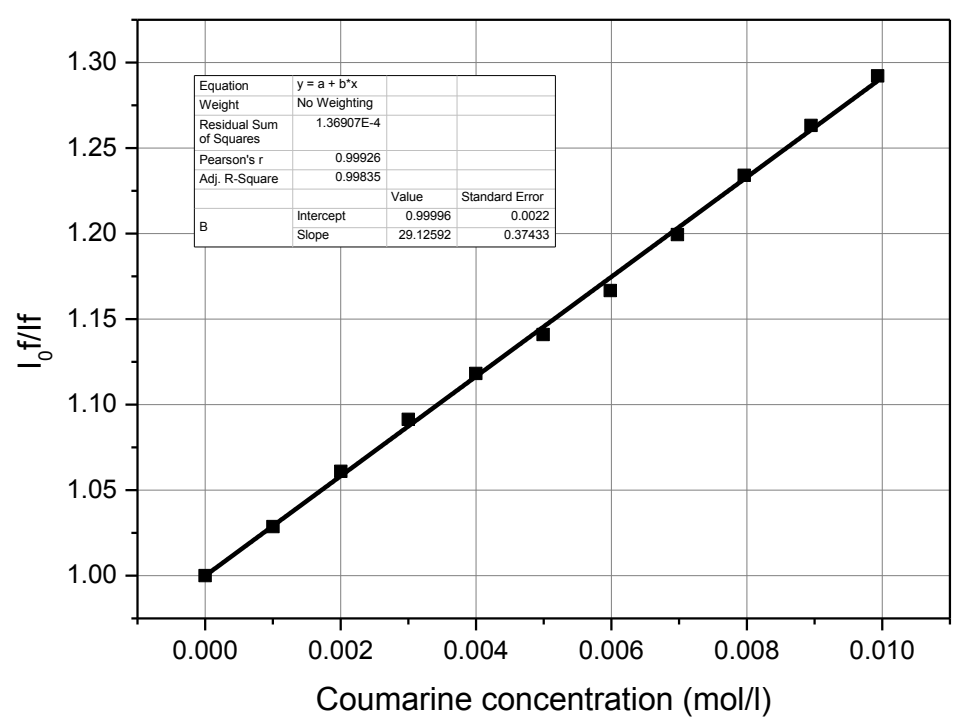

Quenching of 2c with 3a

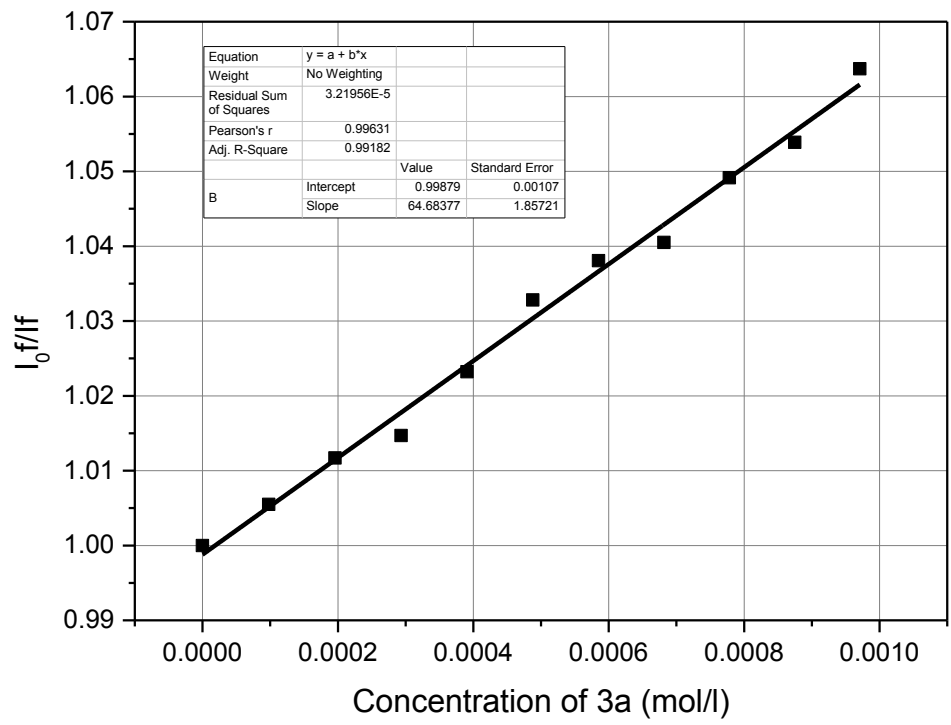


S8 Experimental setup for cycloreversion reaction under sunlight

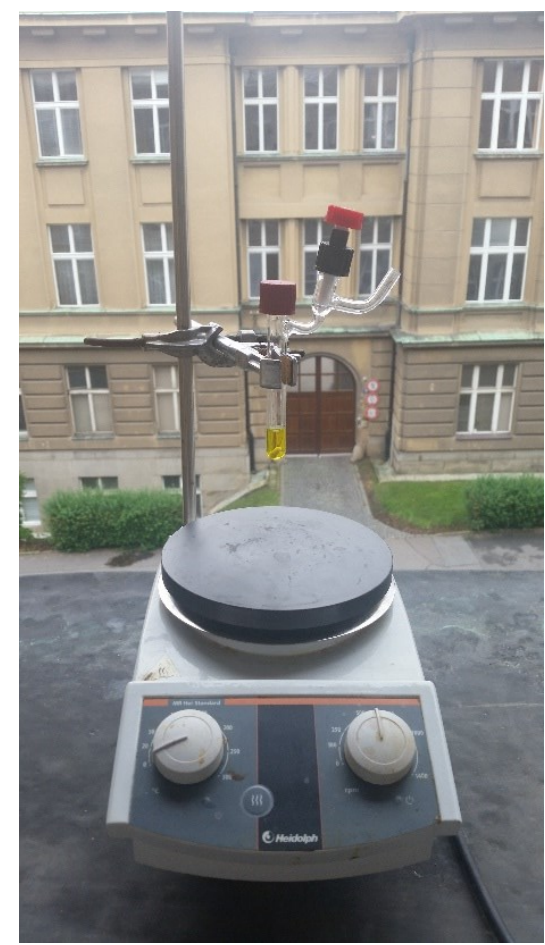

Cycloreversion experiment using sunlight: Prague, Czech Republic, 19.5.2016 9:30-9:45 am. GPS: N 50.101728, E 014.389929. 
S9 Effect of oxygen concentration on cycloreversion reaction with 2c

\begin{tabular}{ccccccc}
\hline \multirow{2}{*}{ Entry } & Cyclobutane & $\begin{array}{c}\text { Time } \\
{[\mathrm{min}]}\end{array}$ & Catalyst & \multicolumn{3}{c}{$\begin{array}{c}\text { Conversion } \\
{[\%]}\end{array}$} \\
\cline { 5 - 7 } & & & & $\begin{array}{c}\text { Deggased } \\
\text { under } \operatorname{Ar}^{a}\end{array}$ & $\operatorname{Air}^{b}$ & $\mathrm{O}_{2}{ }^{c}$ \\
\hline 1 & $\mathbf{3 b}$ & 2 & $\mathbf{2 c}$ & 49 & 45 & 47 \\
2 & $\mathbf{3 f}$ & 4 & $\mathbf{2 c}$ & 79 & 79 & 85 \\
\hline
\end{tabular}

Reaction conditions: $3 \mathbf{b}(0.02 \mathrm{mmol})$, photocatalyst $(2.5 \mathrm{~mol} \%)$, solvent $(1 \mathrm{~mL})$, visible light, $\mathrm{rt}$.

${ }^{a}$ Three-times degassed using freeze-pump-thaw technique; ${ }^{b}$ Experiment under air; ${ }^{c}$ Bubbled with oxygen $(2 \mathrm{~min})$ before irradiation. 
S10 Cycloreversion of isomeric pyrimidine dimers

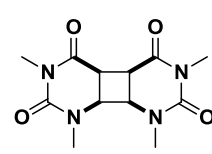

3f

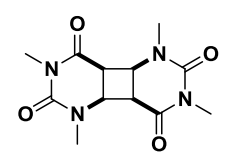

$3 f a$

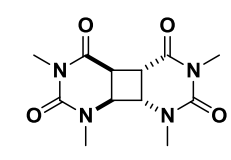

$3 \mathrm{fb}$

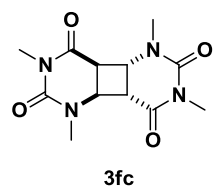

$3 \mathrm{fc}$

$$
\begin{gathered}
2 c \\
450 \mathrm{~nm}
\end{gathered}
$$

$$
\text { if }
$$

\begin{tabular}{ccc}
\hline Entry & Cyclobutane & $\begin{array}{c}\text { Conv. }(3 \mathrm{~min}) \\
{[\%]}\end{array}$ \\
\hline 1 & $\mathbf{3 f}$ & 30 \\
2 & $\mathbf{3 f a}$ & 17 \\
3 & $\mathbf{3 f b}$ & 20 \\
4 & $\mathbf{3 f c}$ & 36 \\
\hline
\end{tabular}

Quantitative conversion was observed within 30 minutes for all isomers 


\section{S11 Stern-Volmer plots for quenching of 3b-cycloreversion catalyzed by 2c}

Reaction conditions: $\mathbf{3 b}$ (0.02 mmol), $\mathbf{2 c}$ (2.5 mol \%), quencher (various concentrations), acetonitrile $(1 \mathrm{~mL})$, visible light $(450 \mathrm{~nm}), \mathrm{rt}, \mathrm{Ar}, 2 \mathrm{~min}$.

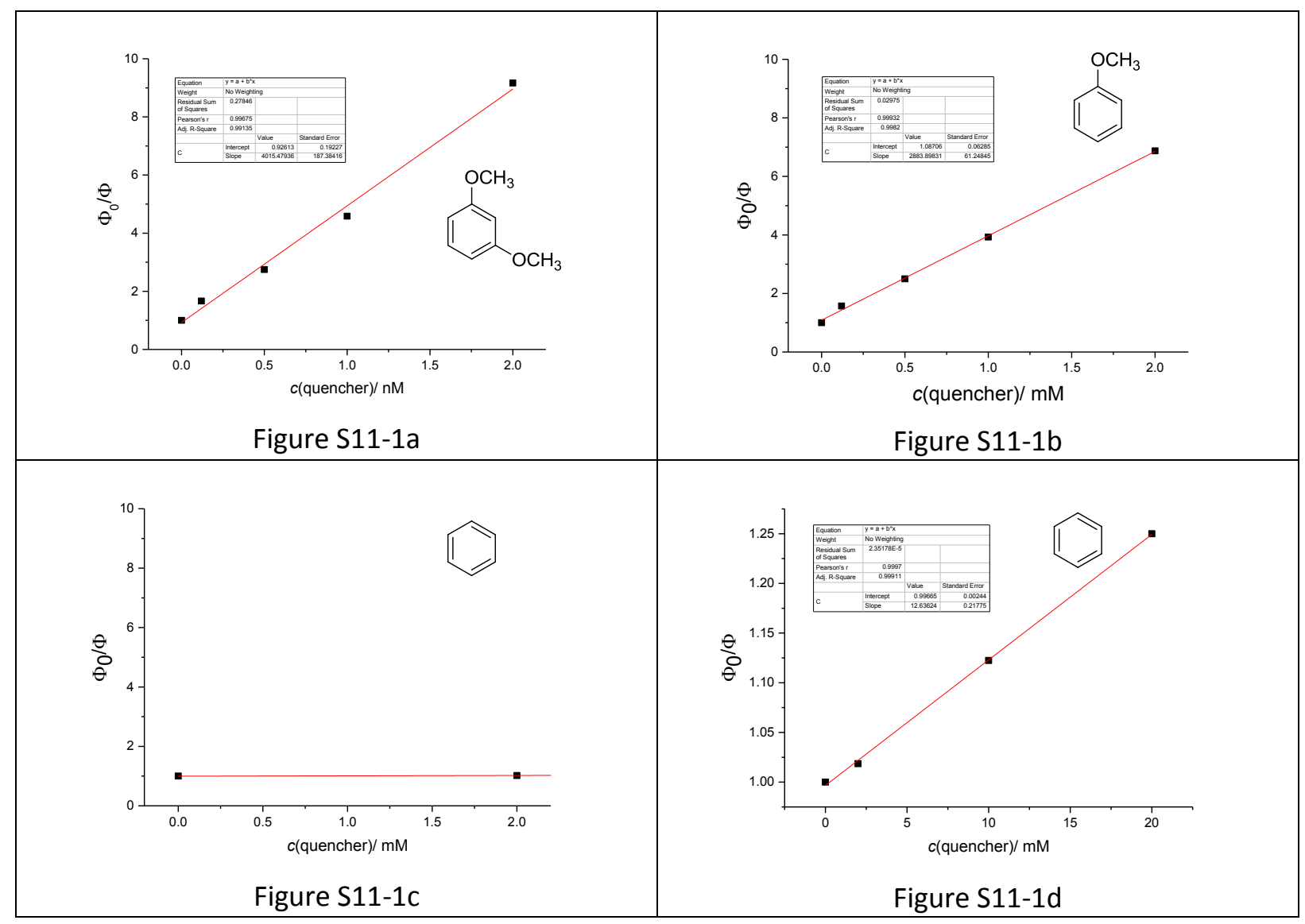

Effective quenching of $\mathbf{3 b}$-photocycloreversion mediated by catalyst $\mathbf{2 c}$ was observed using quenchers (anisole and 1,3-dimethoxybenzene) with lower oxidation potentials ${ }^{11}$ compared to $3 \mathbf{b}\left(E^{\mathrm{ox}}=2.05 \mathrm{~V}\right.$ vs $\mathrm{SCE})$. On the other hand benzene ${ }^{12}$ having higher oxidation potential did not almost cause quenching of photocycloreversion (see Figure S11-2).

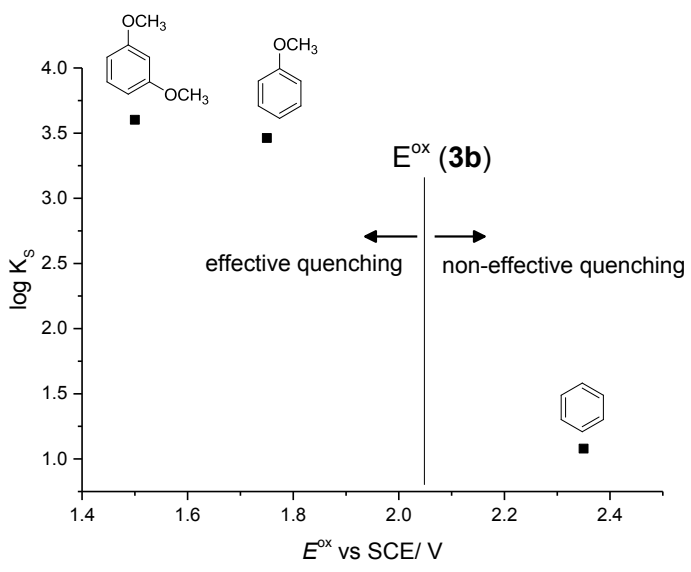

Figure S11-2

\footnotetext{
${ }^{11}$ Porcal, G.; Bertolotti, S. G.; Previtali, C. M.; Encinas, M. V. Phys. Chem. Chem. Phys. 2003, 5, 4123.

${ }^{12}$ Fukuzumi, S.; Okhubo, K.; Suenobu, T.; Kato, K.; Fujitsuka, M.; Ito, O. J. Am. Chem. Soc. 2001, 123, 8459.
} 


\section{S12 Copy of NMR Spectra of compounds 3}

$3 a$

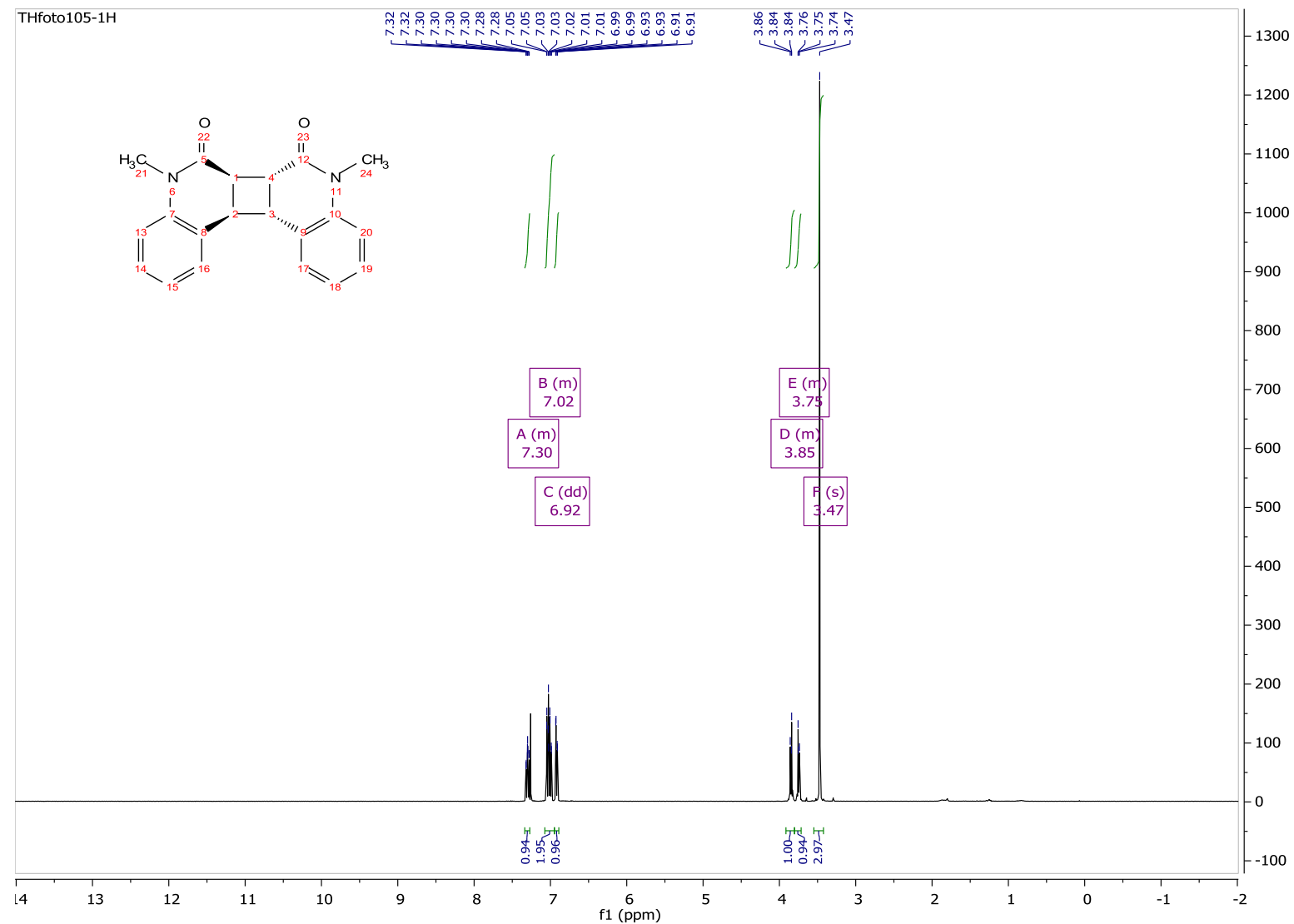

THfoto105-13C

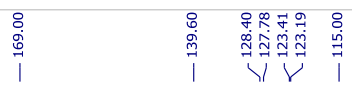

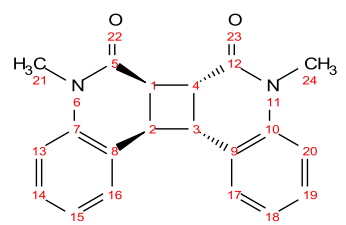

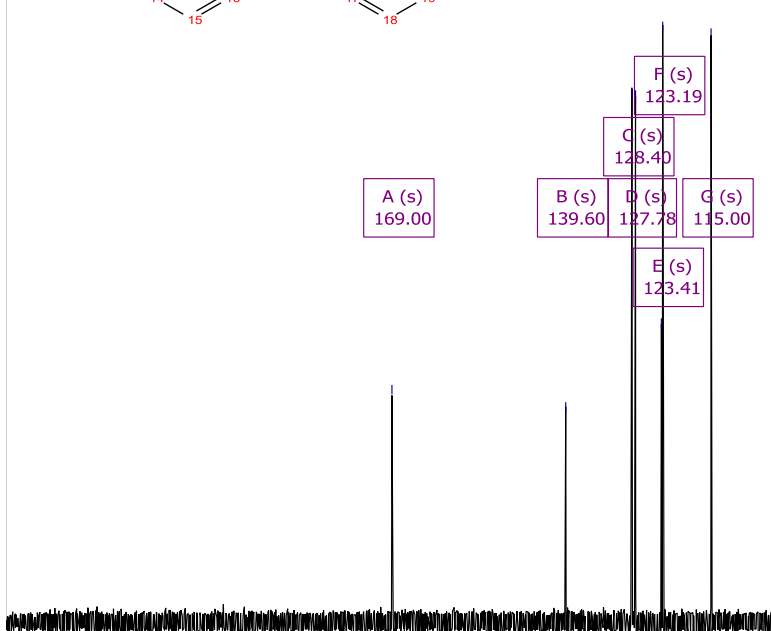

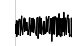

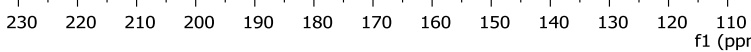


3b
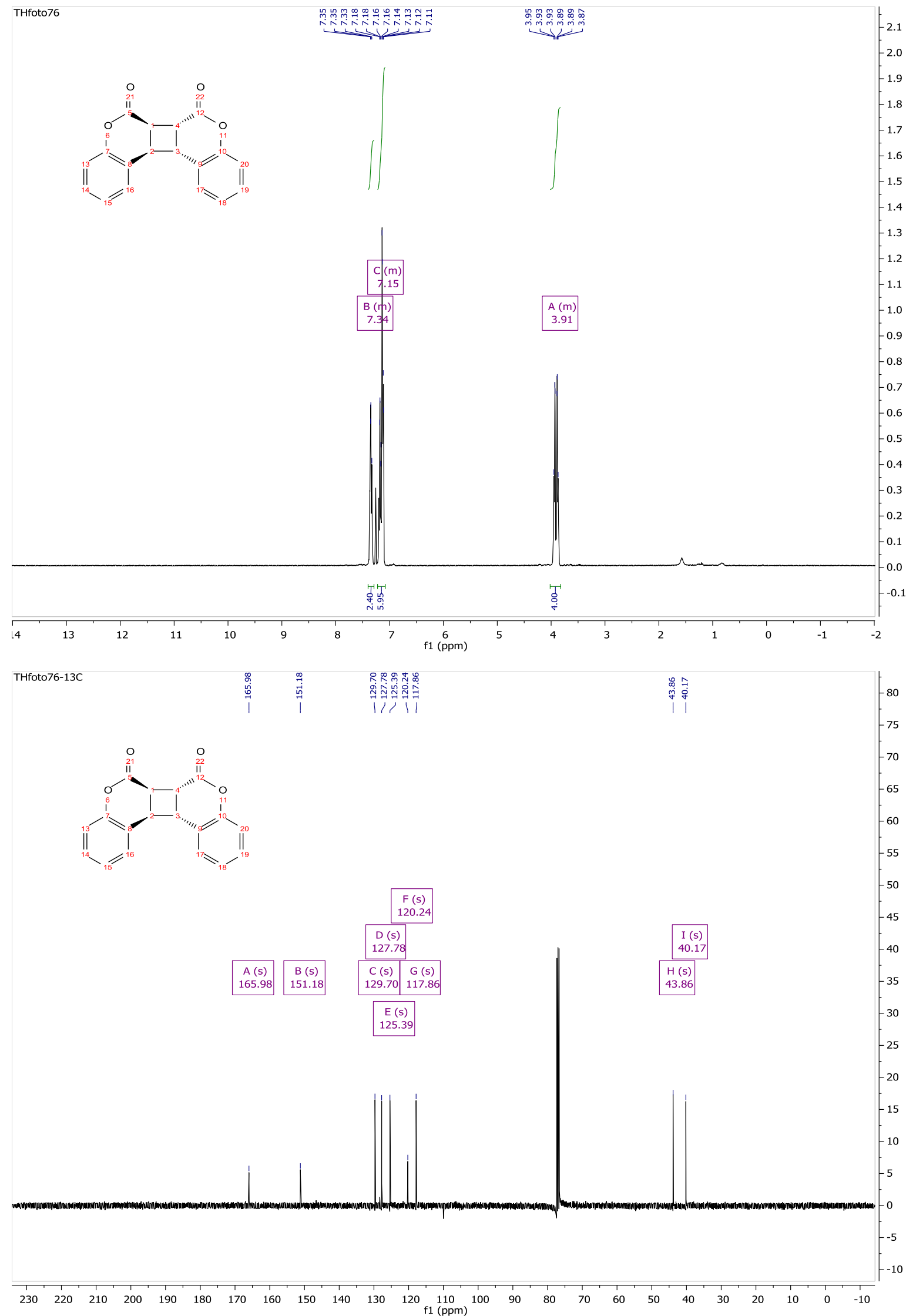
3c

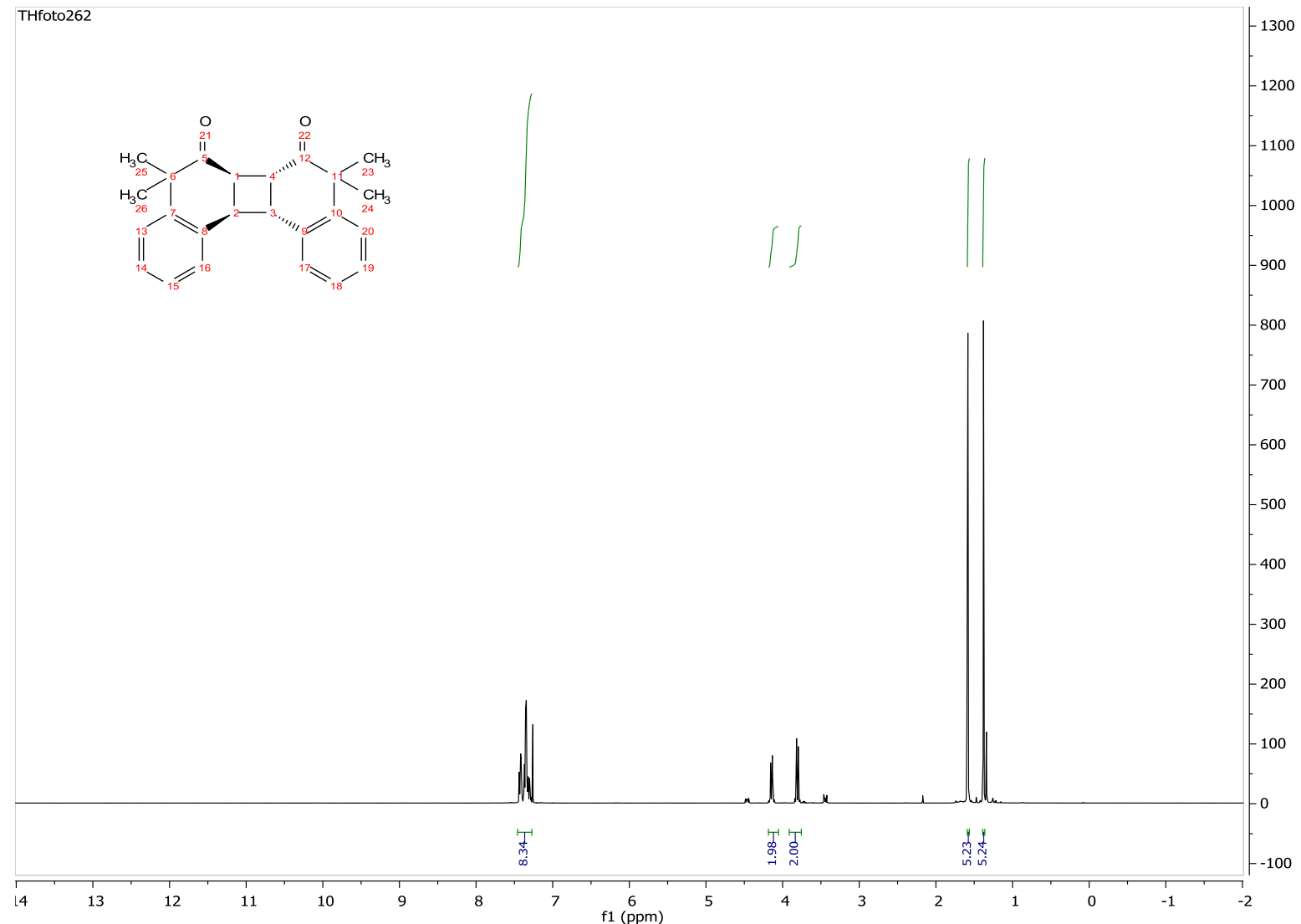

THfoto262-13C : DEUTERIUM OB唯RVE STANDARD PARAMMETERS

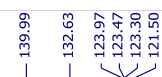

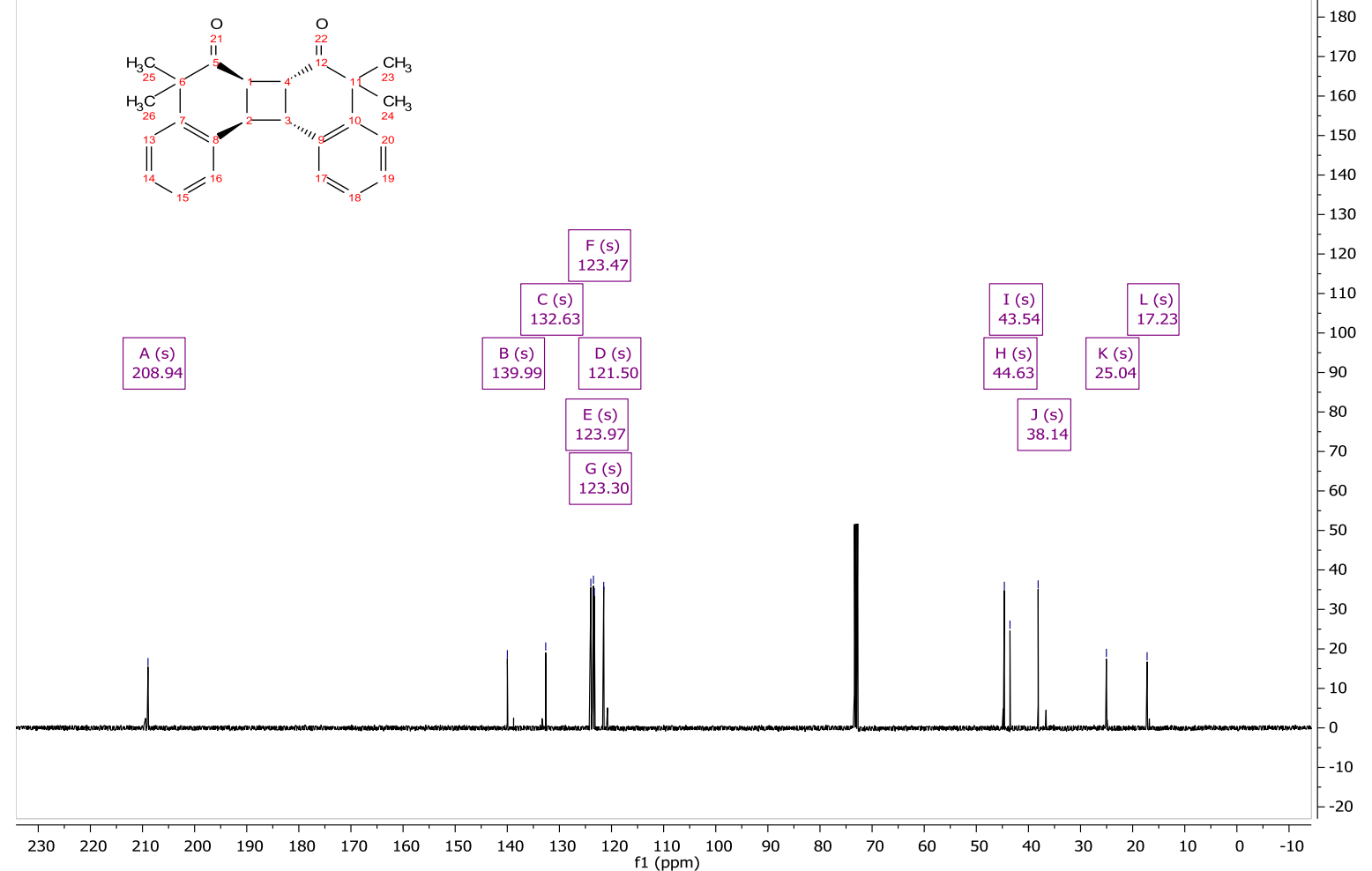


3d
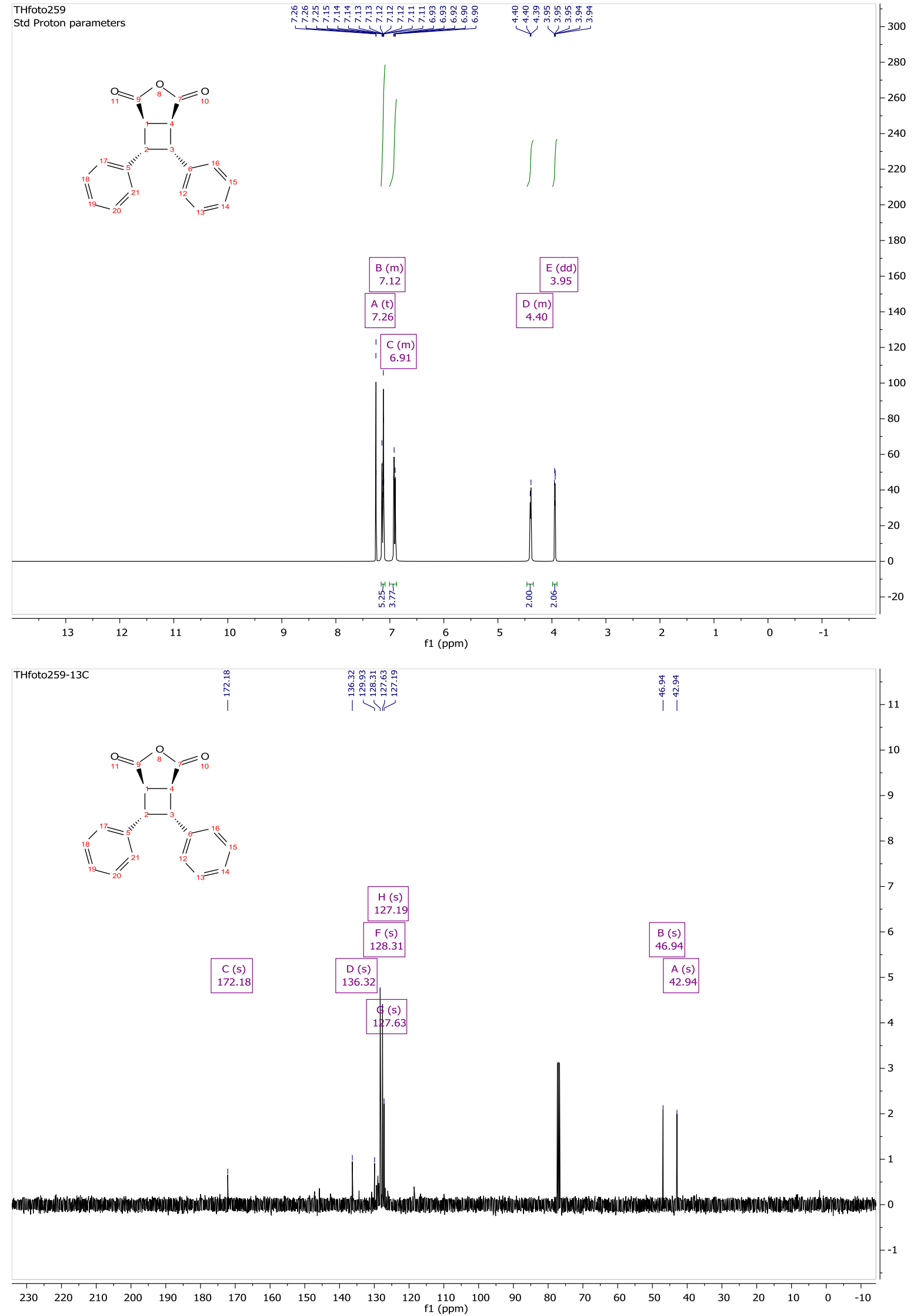
$3 e$

THfoto282-1H

Selective band center: $5.85(\mathrm{ppm})$; width: $11.5(\mathrm{~Hz})$

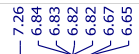

Selective band center: $5.26(\mathrm{ppm})$; width: $11.2(\mathrm{~Hz})$<smiles></smiles>
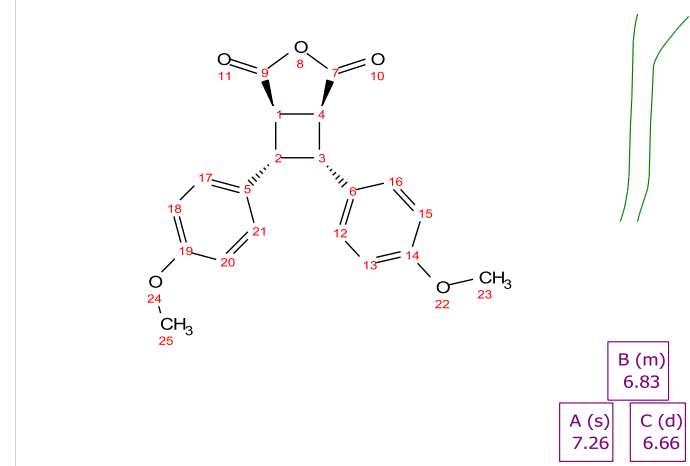

\begin{tabular}{l}
$3(m)$ \\
6.83 \\
\hline$C(d)$
\end{tabular}

$7.26 \quad 6.66$
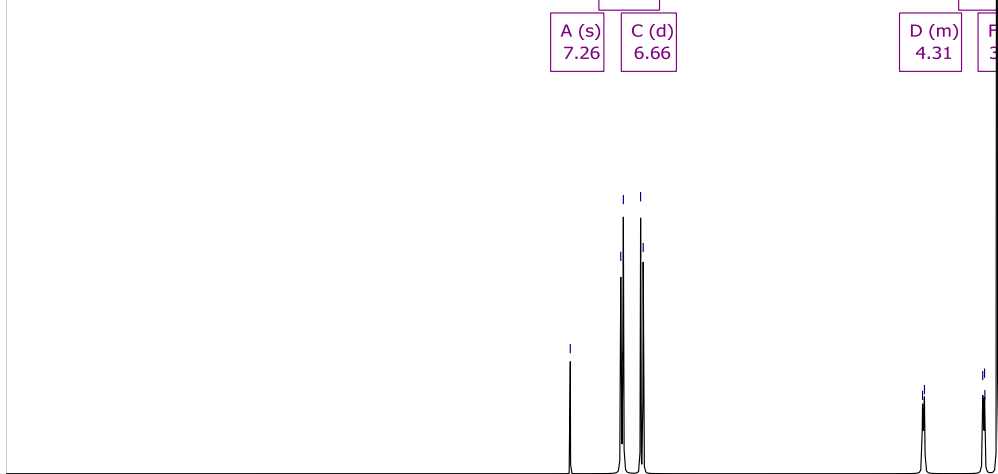

雾点

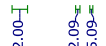

$\begin{array}{lllllllllllll}11.5 & 11.0 & 10.5 & 10.0 & 9.5 & 9.0 & 8.5 & 8.0 & 7.5 & 7.0 & 6.5 & 6.0 & 5.5 \\ \mathrm{f} 1(\mathrm{ppm})\end{array}$

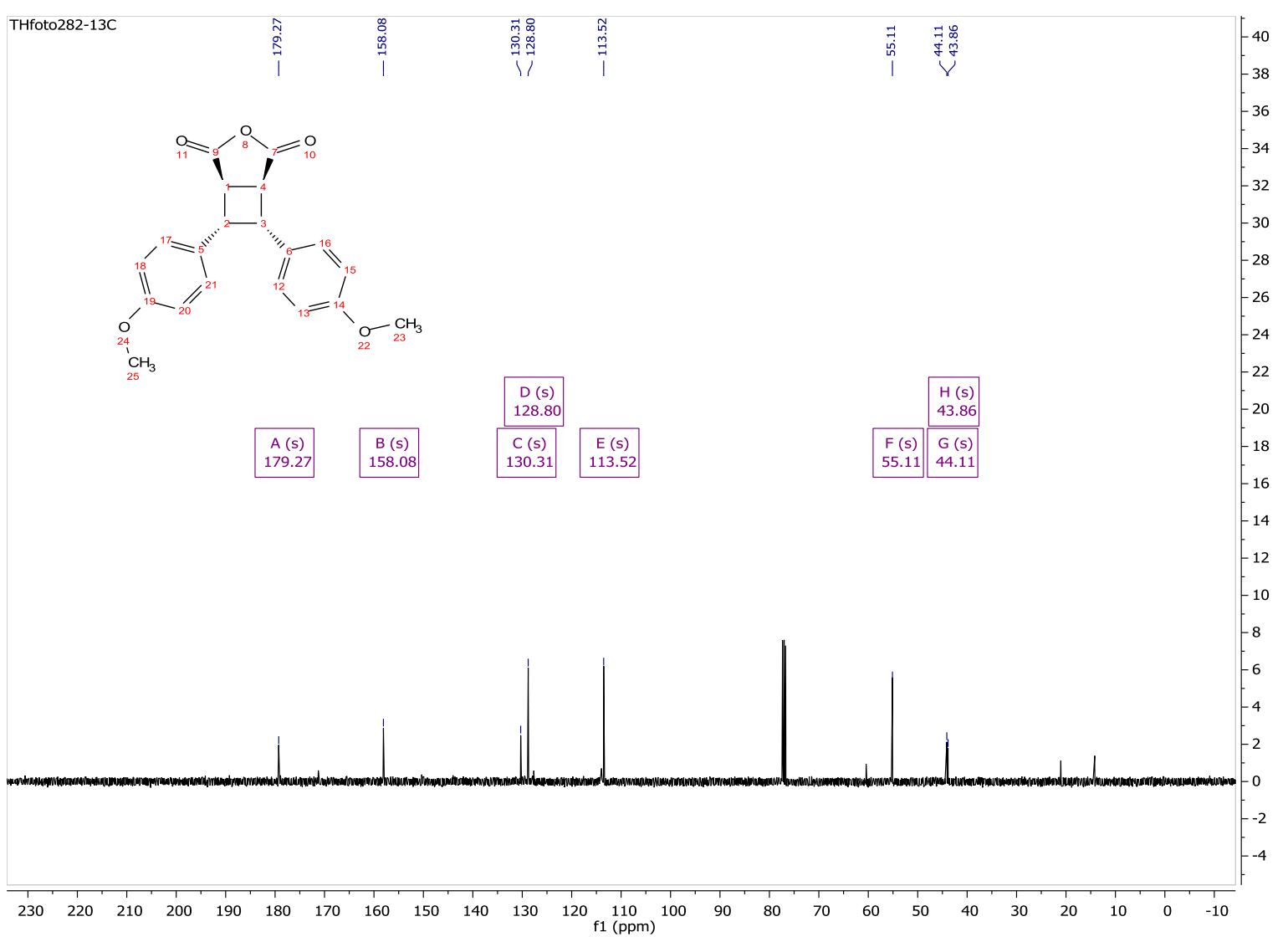




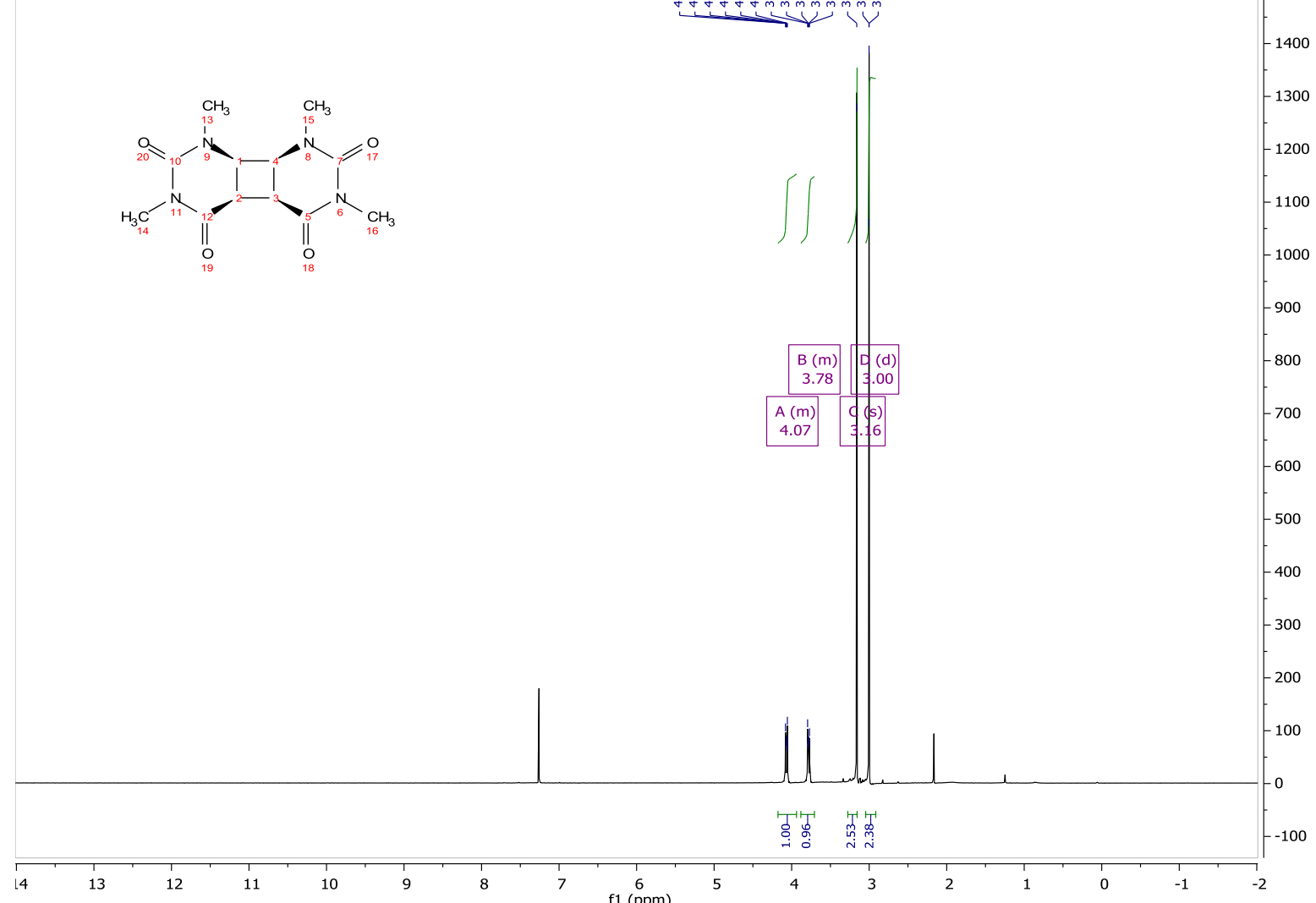

THfoto89-chroum-1-isomer-13C 268_k

壹

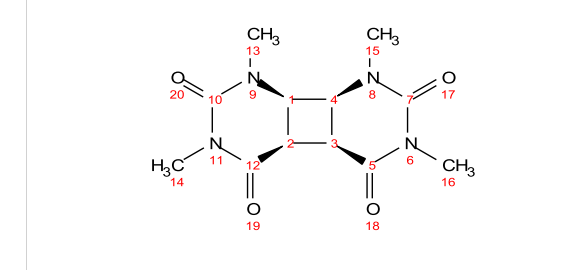

f1 (ppm) ।

$$
\begin{array}{|c|c|}
\hline A(s) & B(s) \\
165.64 & 152.63 \\
\hline
\end{array}
$$

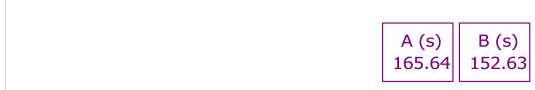

$\begin{array}{llllllllllll}230 & 220 & 210 & 200 & 190 & 180 & 170 & 160 & 150 & 140 & 130 & 120 \\ \mathrm{f} 1(\mathrm{ppm}) & 110 & 10\end{array}$ 
$3 g$

THfoto111-chroum Std Proton parameters<smiles>CN1C(=O)N(C)[C@H]2[C@H]1[I](C)(C)P2(C)(C)C</smiles>

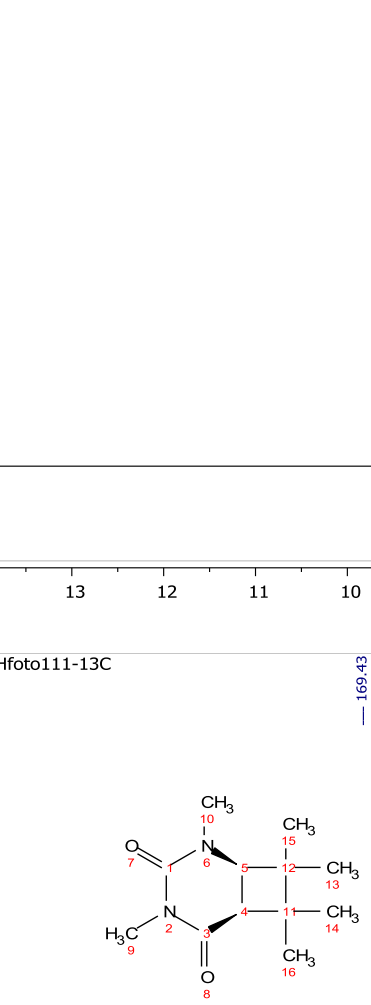

THfoto111-13C

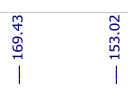

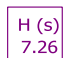

$\begin{array}{r}H(s) \\ 7.26 \\ \hline\end{array}$

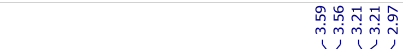

บิำ

Mุi

600

550

500

$-450$

400

300

250

200

50

100

6
$\mathrm{f} 1(\mathrm{ppm})$

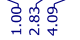

इलंखित
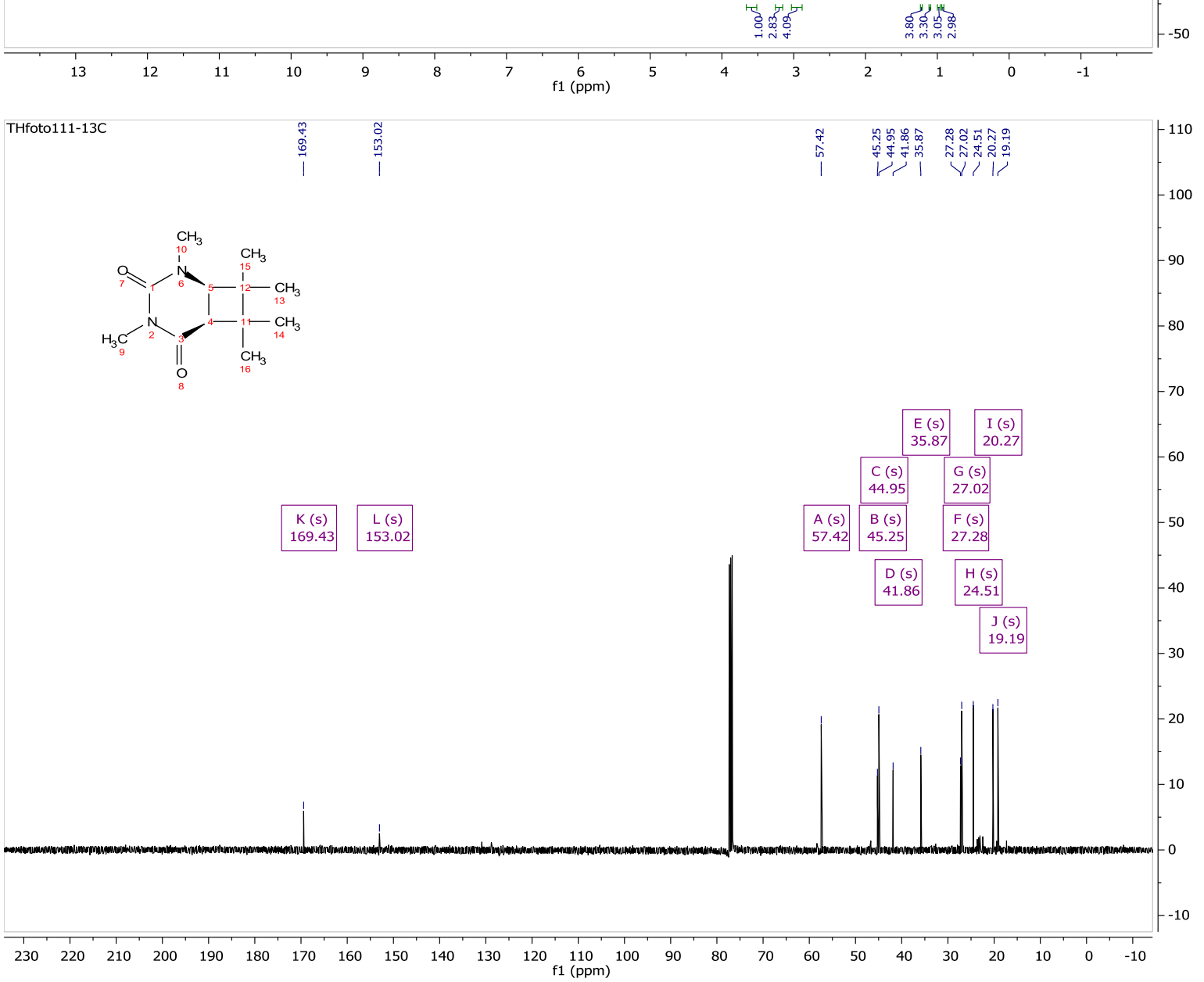
3h
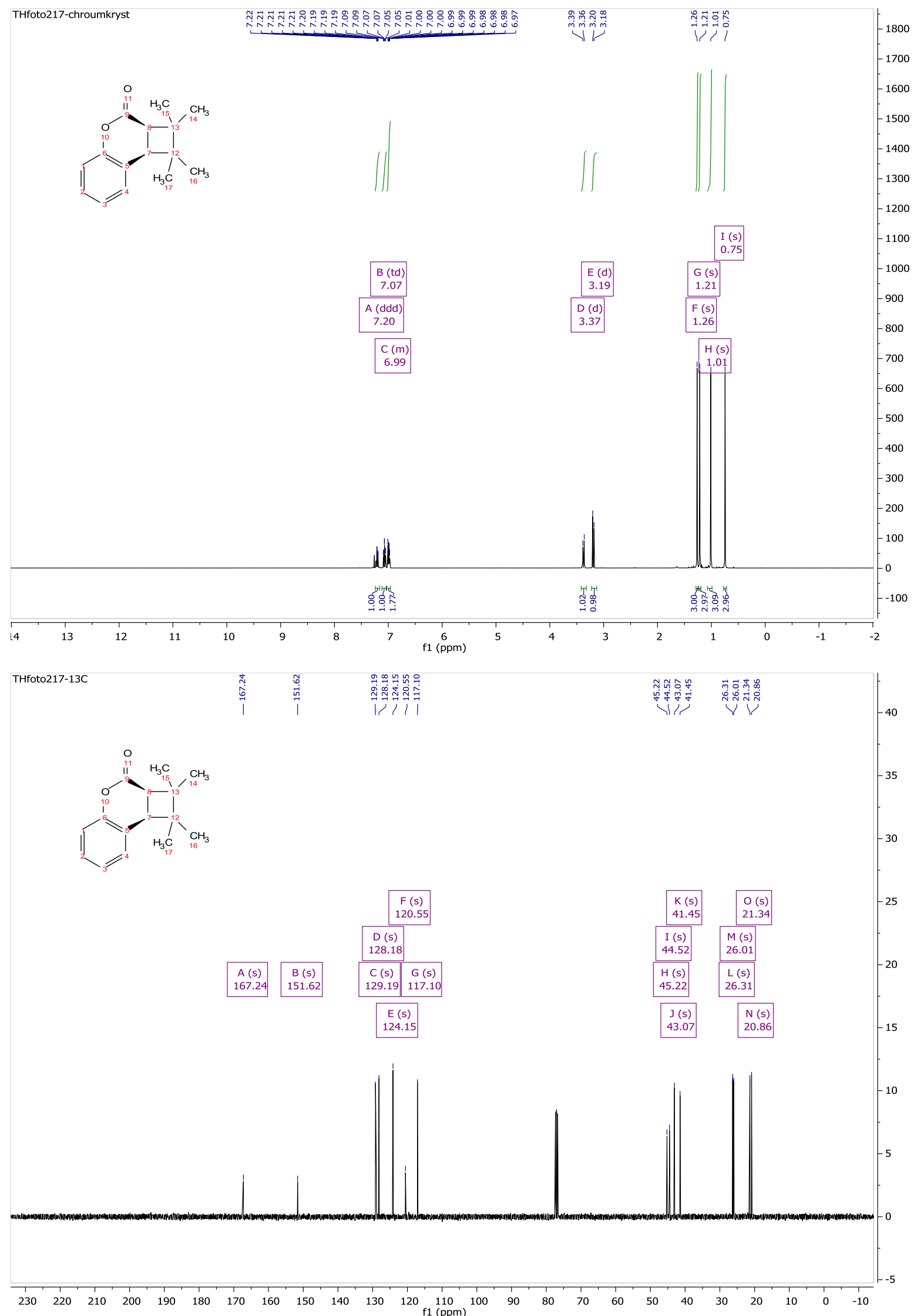

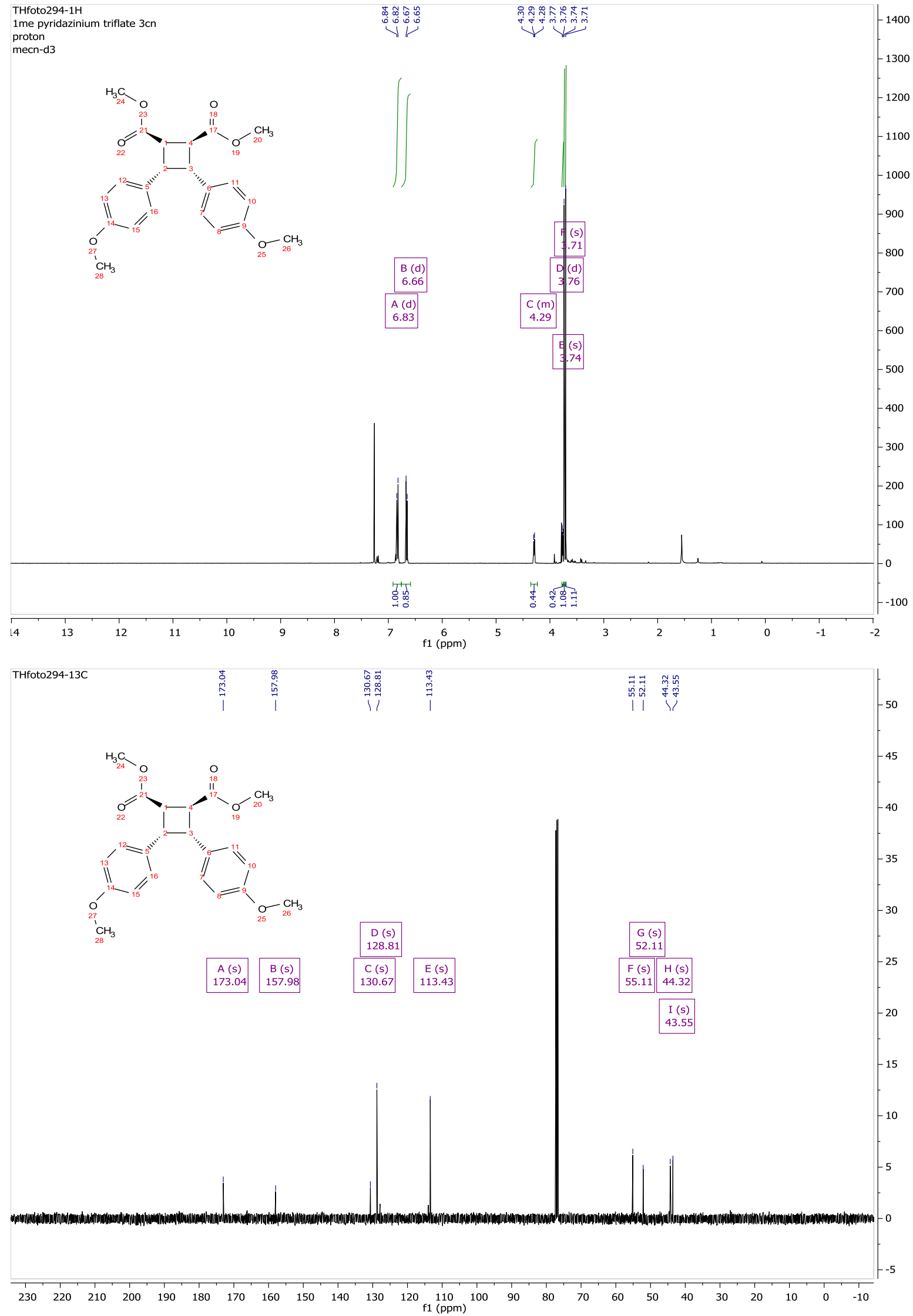
3j
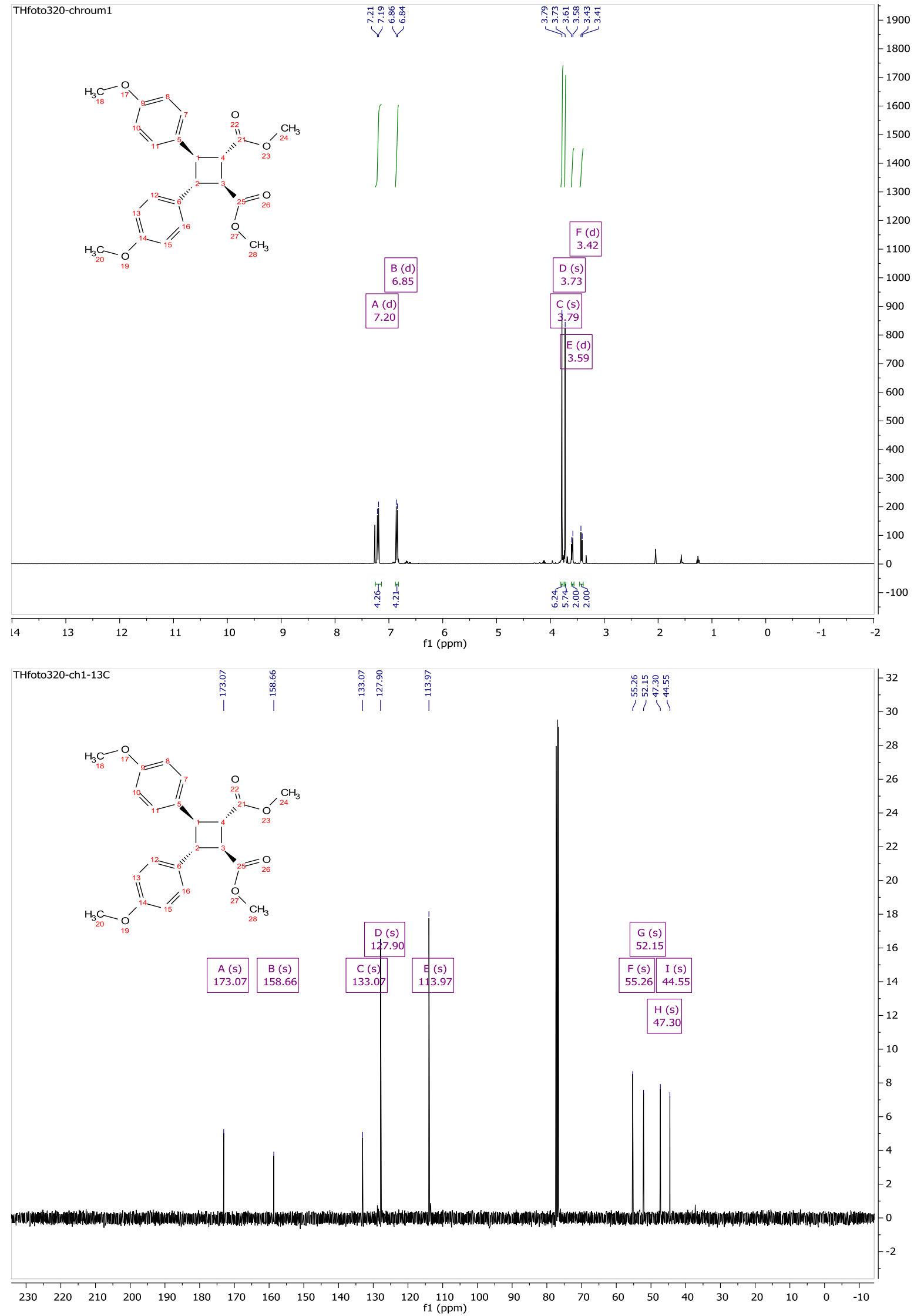
3k
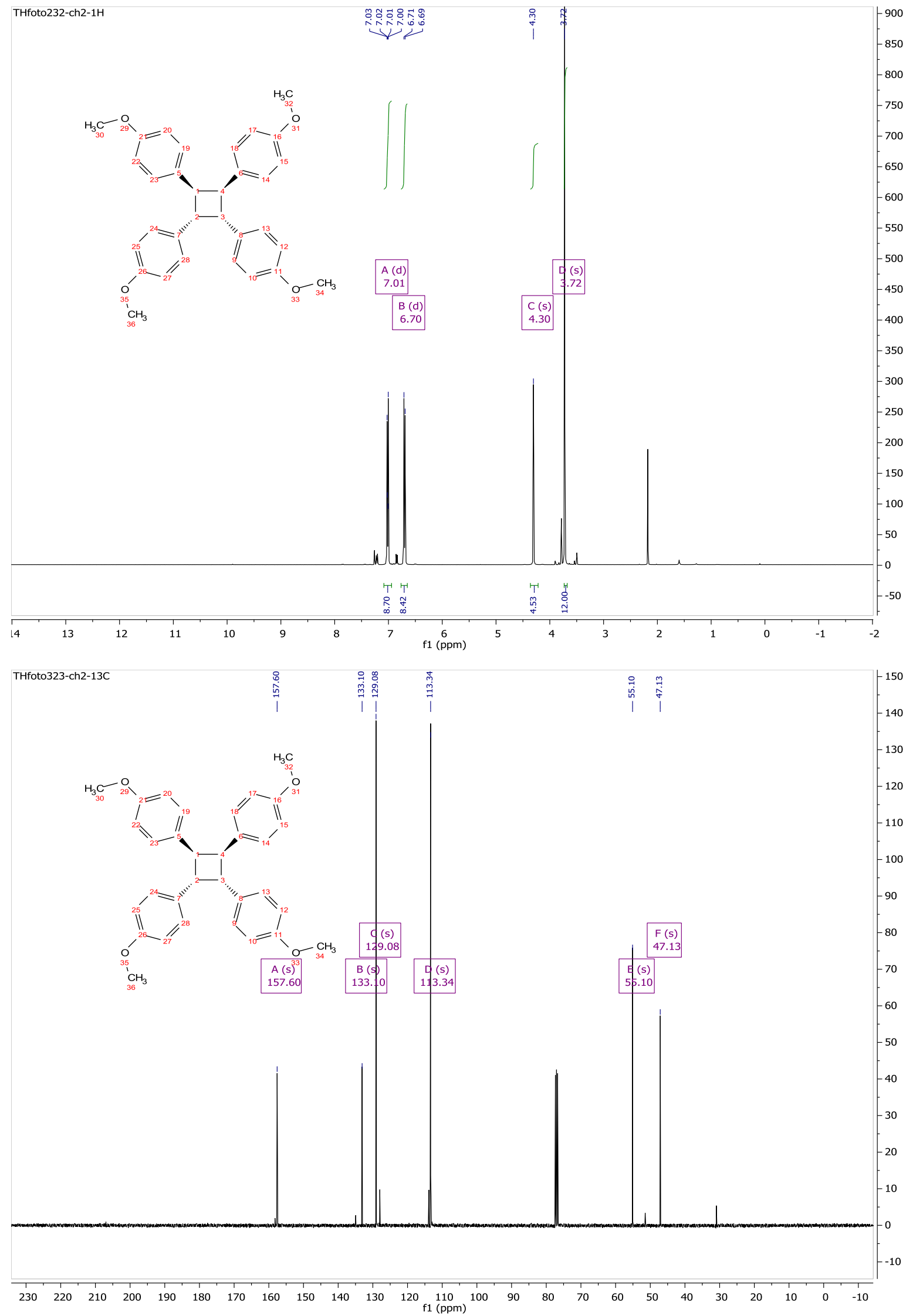
31

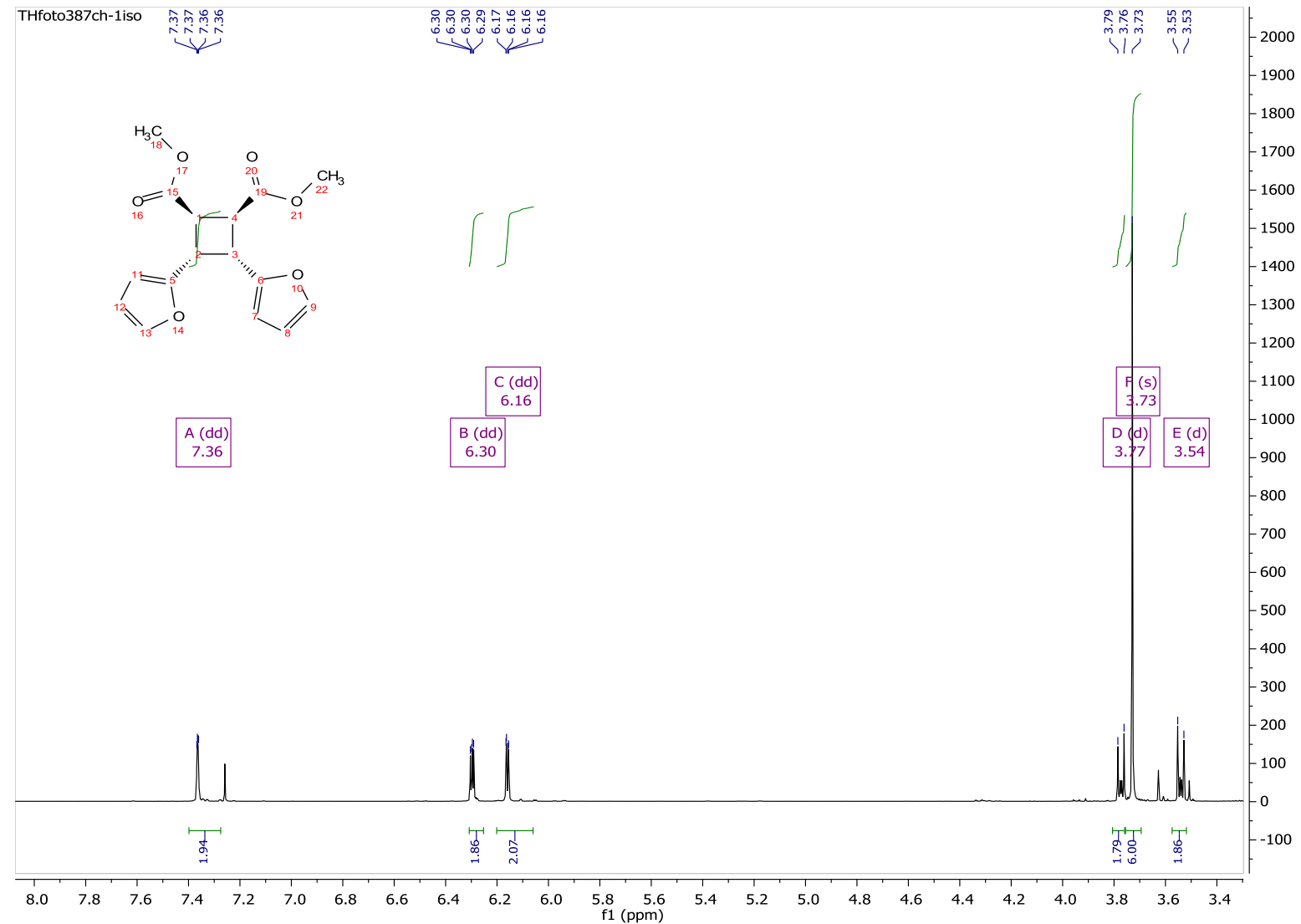

THfoto387-1iso-13C

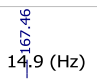

$\stackrel{\substack{n \\ 0}}{\substack{\tilde{m} \\ 0}}$

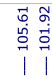

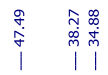<smiles></smiles>
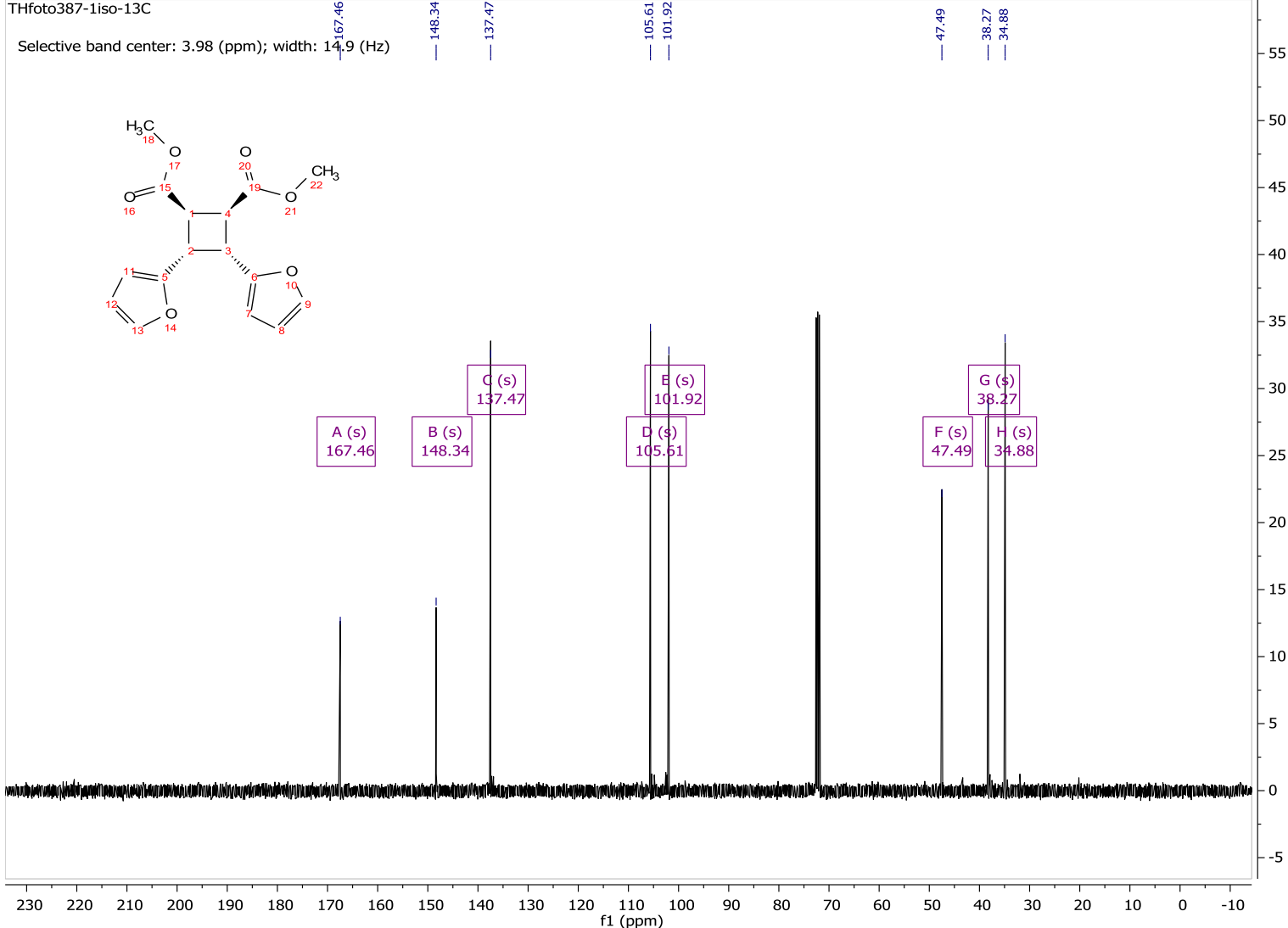


\section{S13 Copy of NMR Spectra of compounds 4}

$4 a$

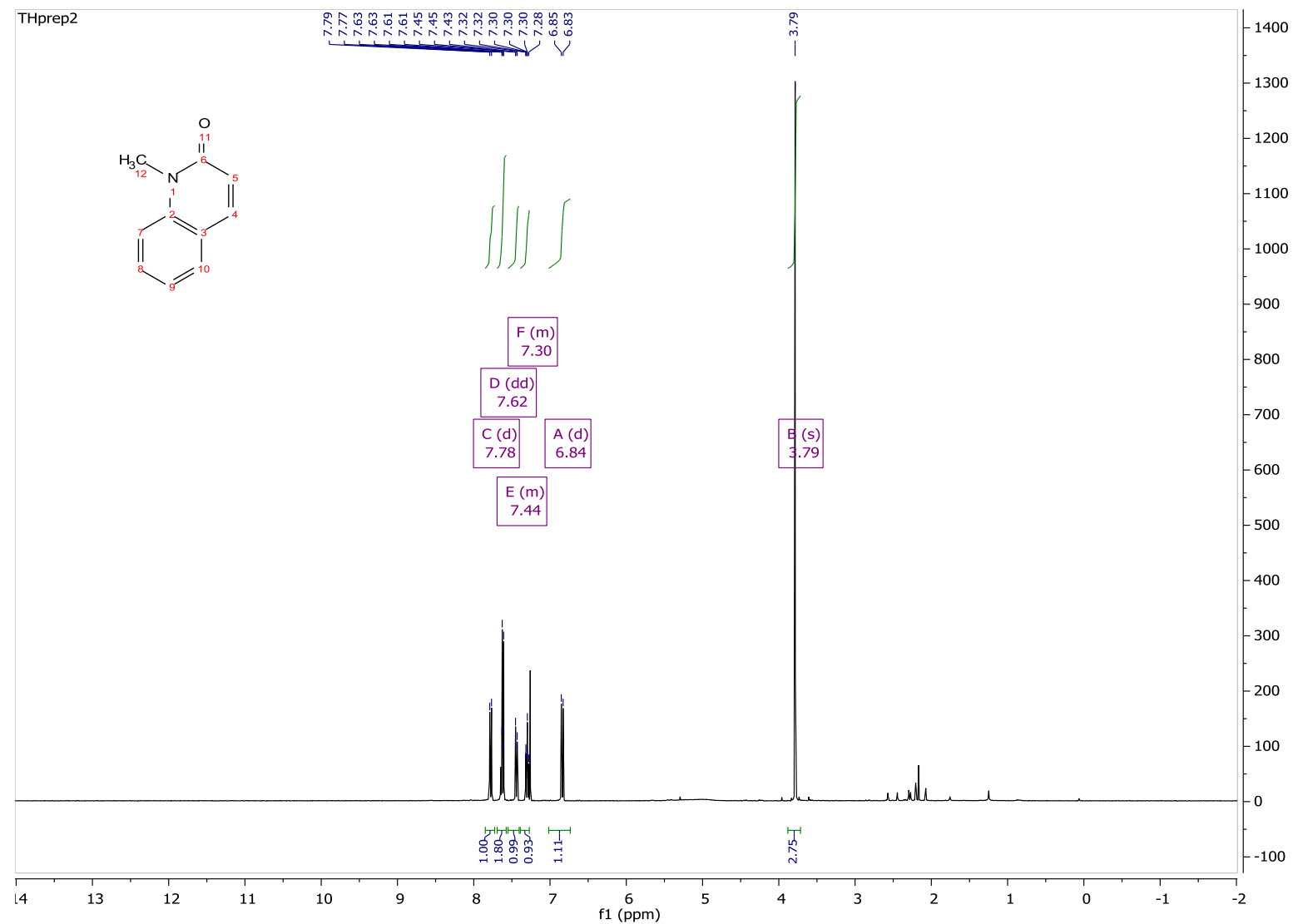

THprep2-13C
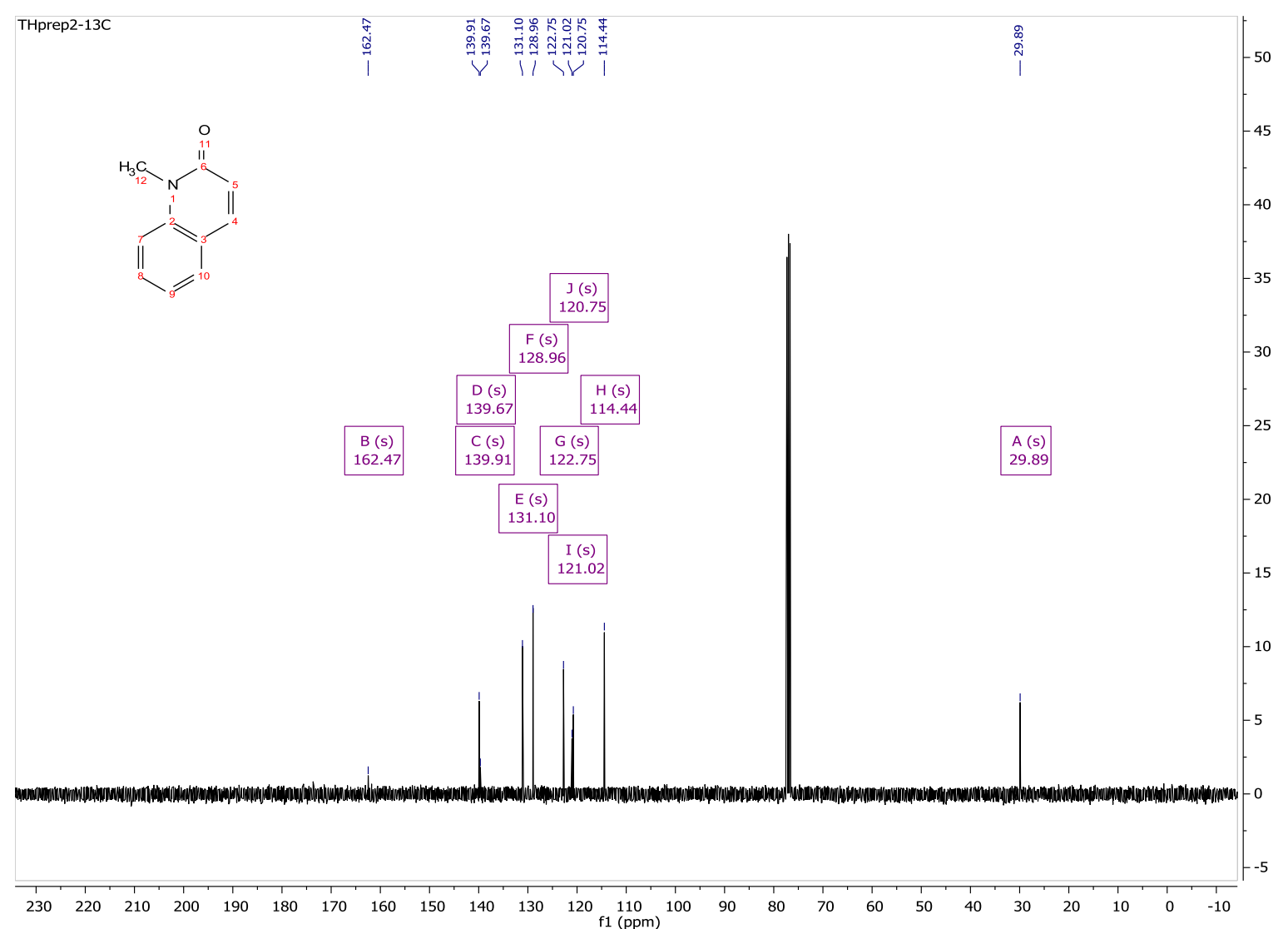
4b
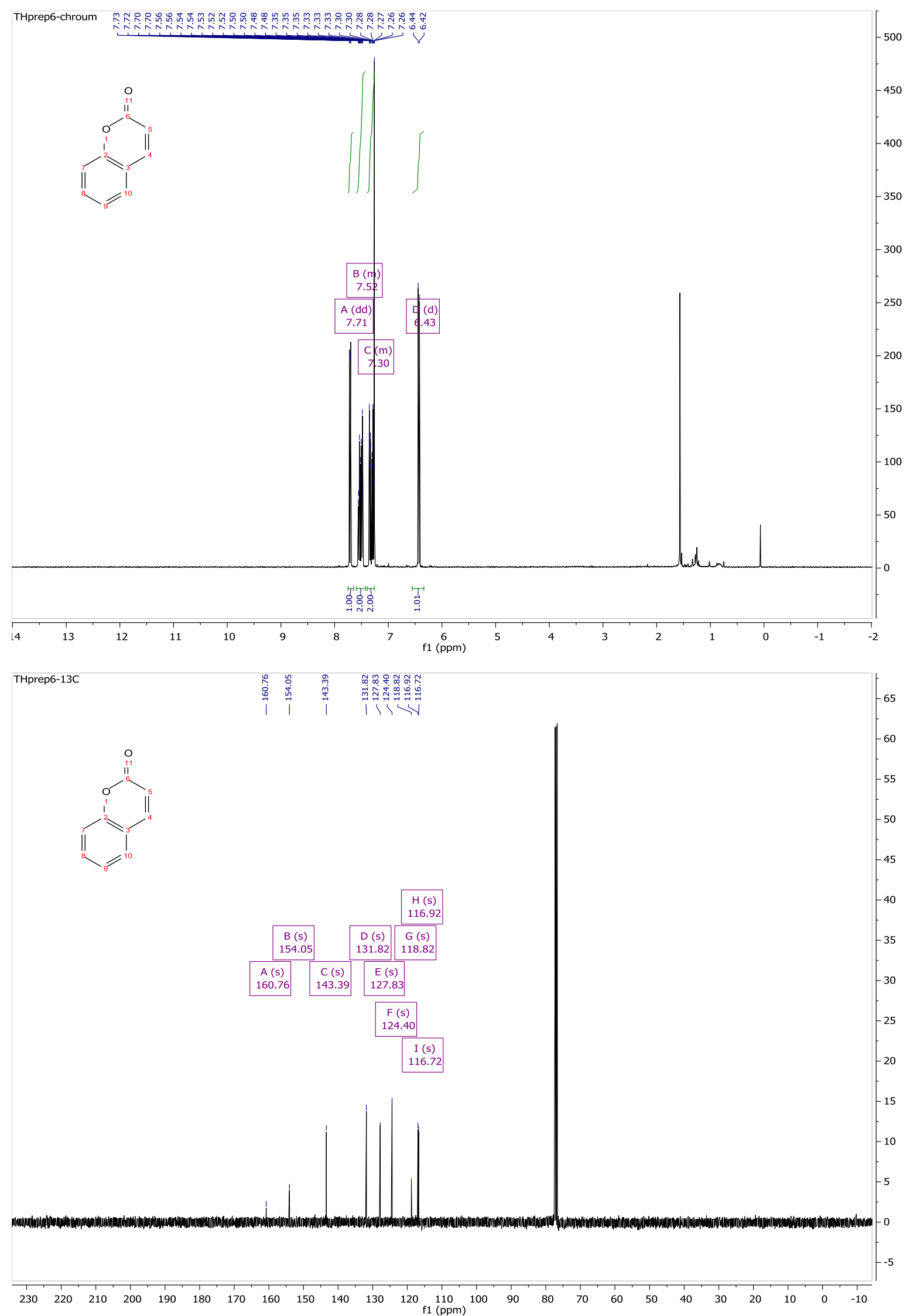
4c
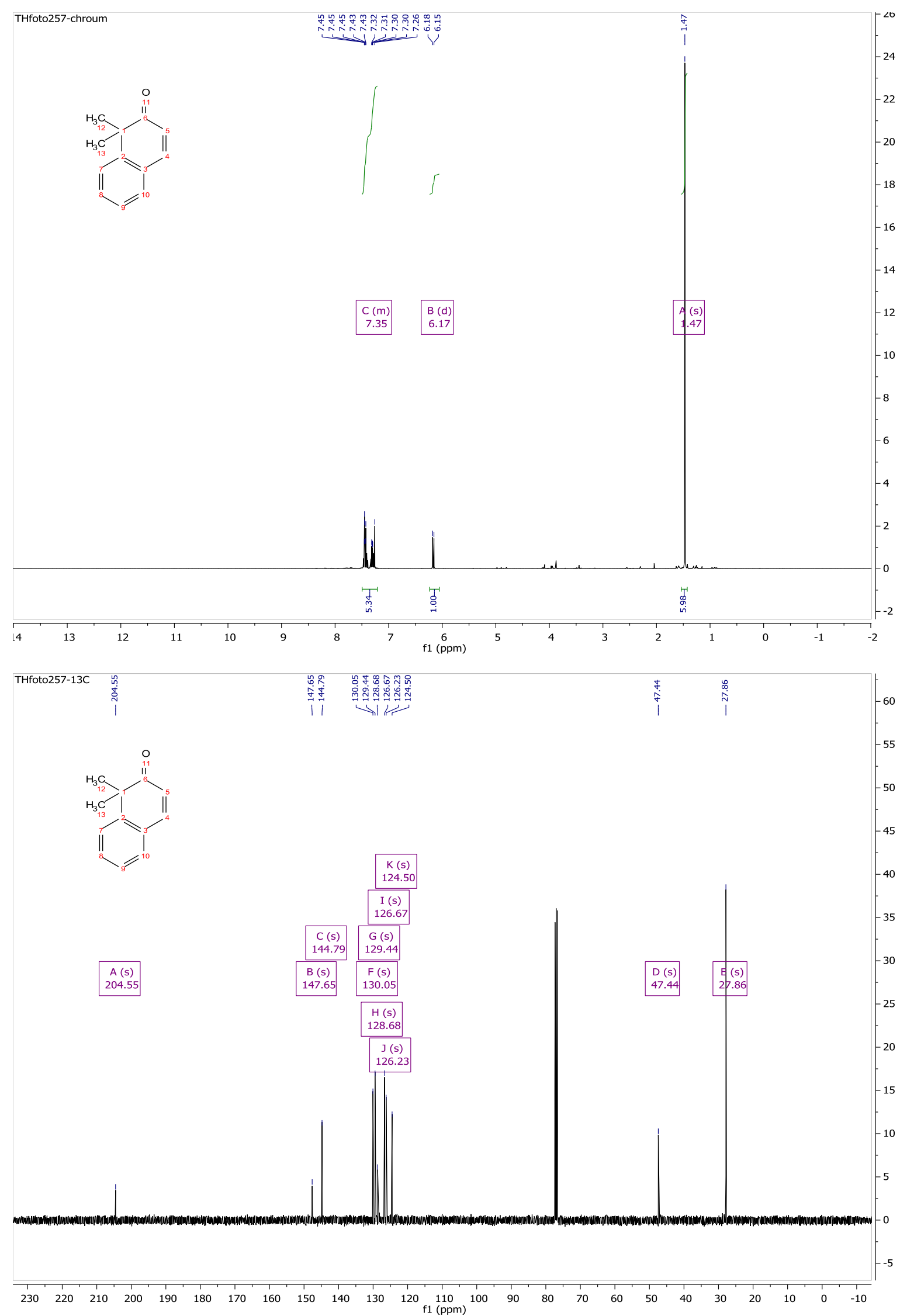
$4 e$
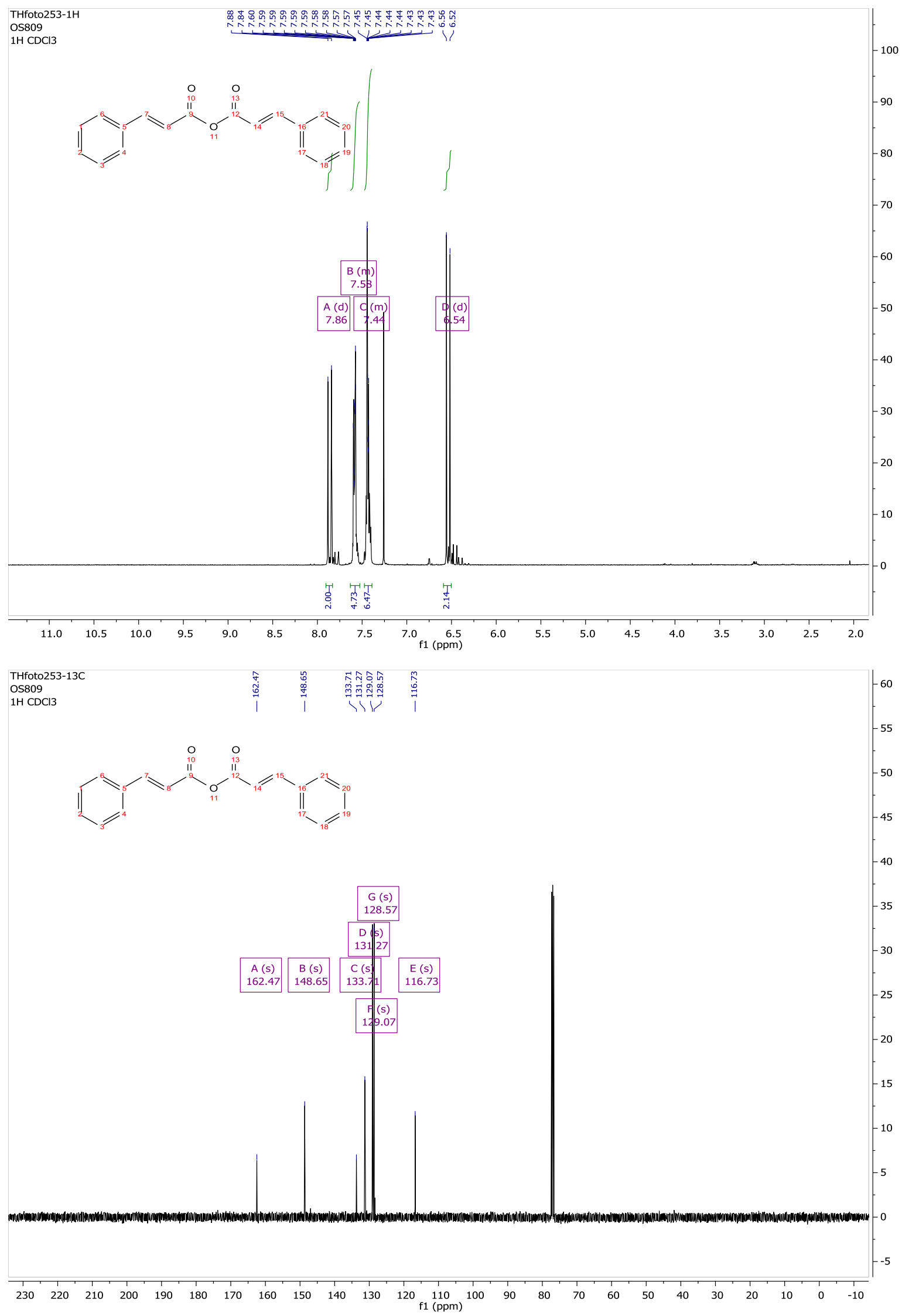

49 

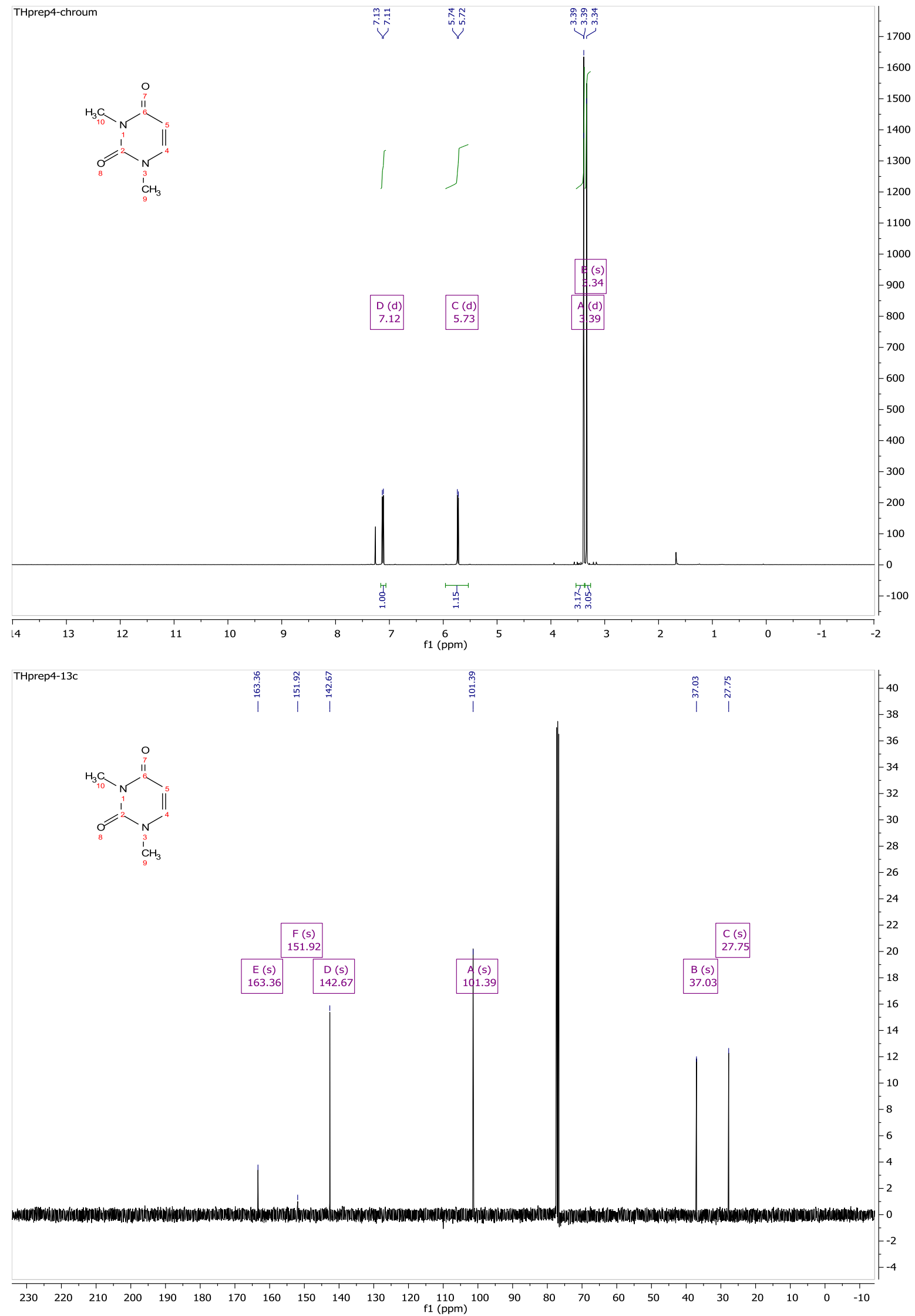
4h

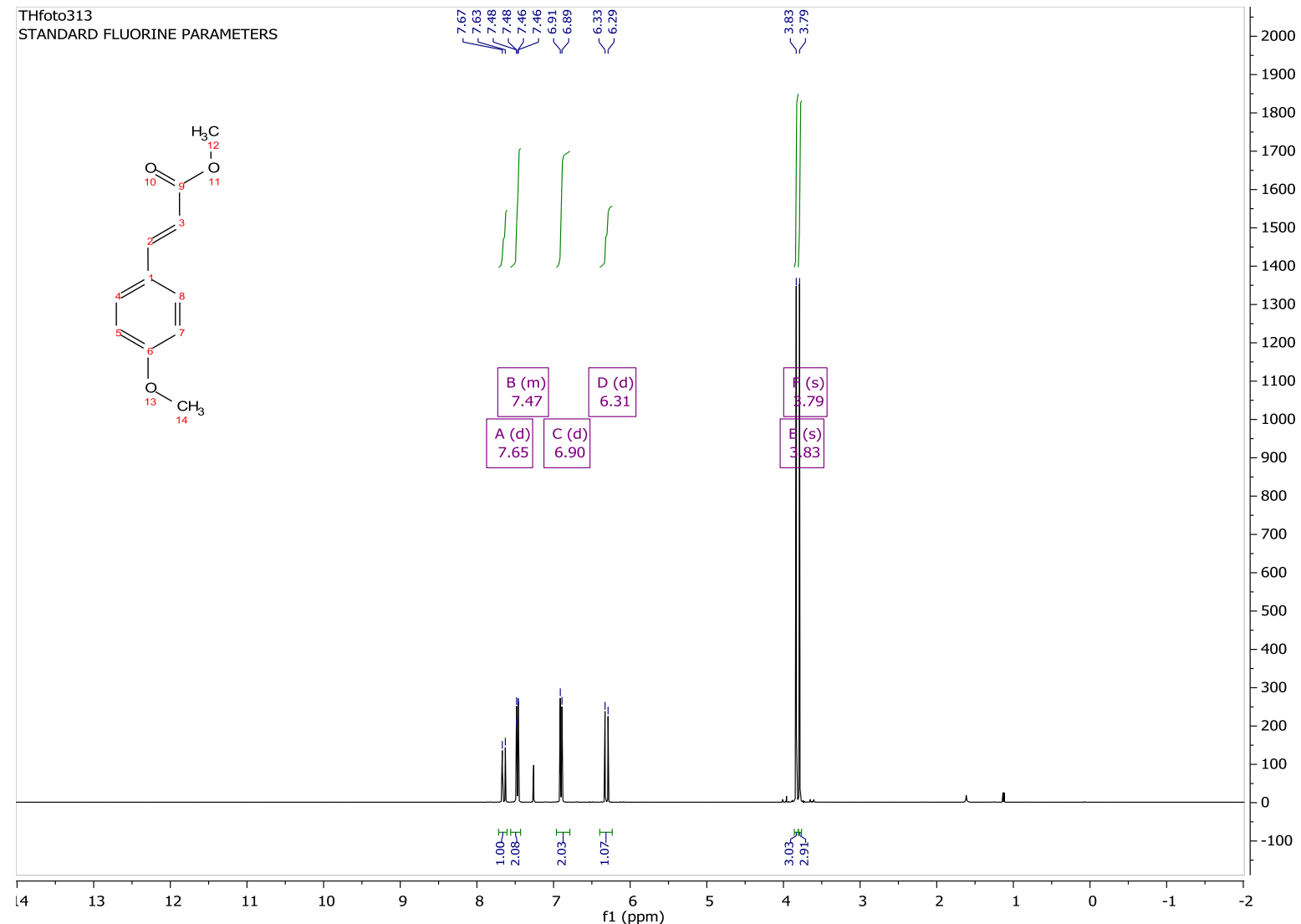

THfoto313-13C

STANDARD FLUORINE PARAMETERS

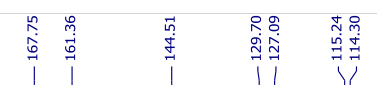
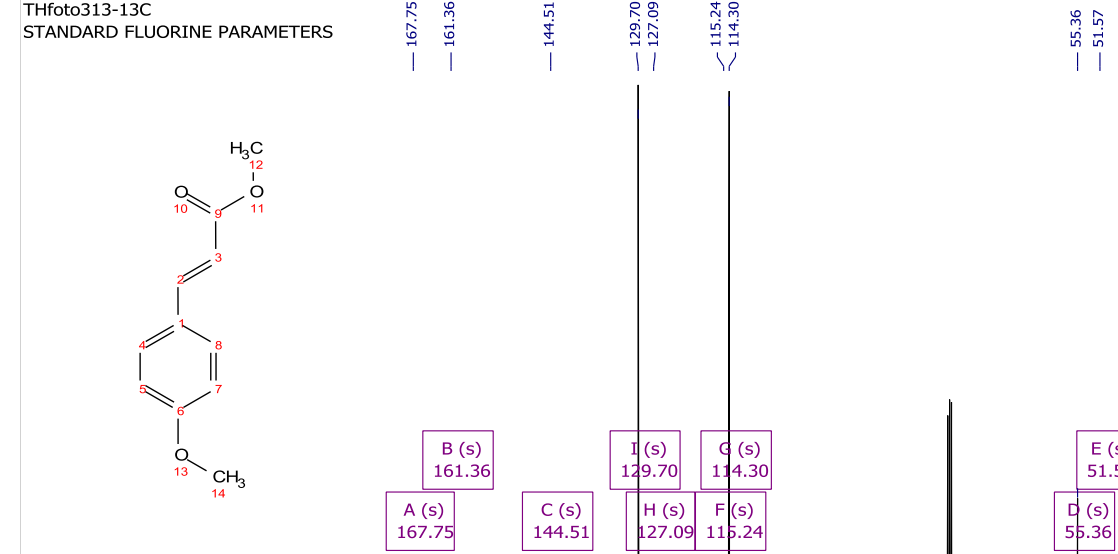

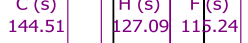

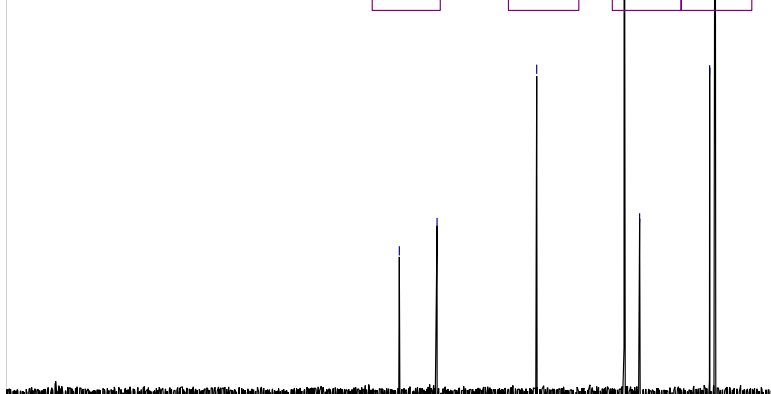

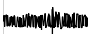

$\begin{array}{llllllllllll}230 & 220 & 210 & 200 & 190 & 180 & 170 & 160 & 150 & 140 & 130 & 120 \\ \mathrm{f} 1(\mathrm{ppm}) & 110\end{array}$ 

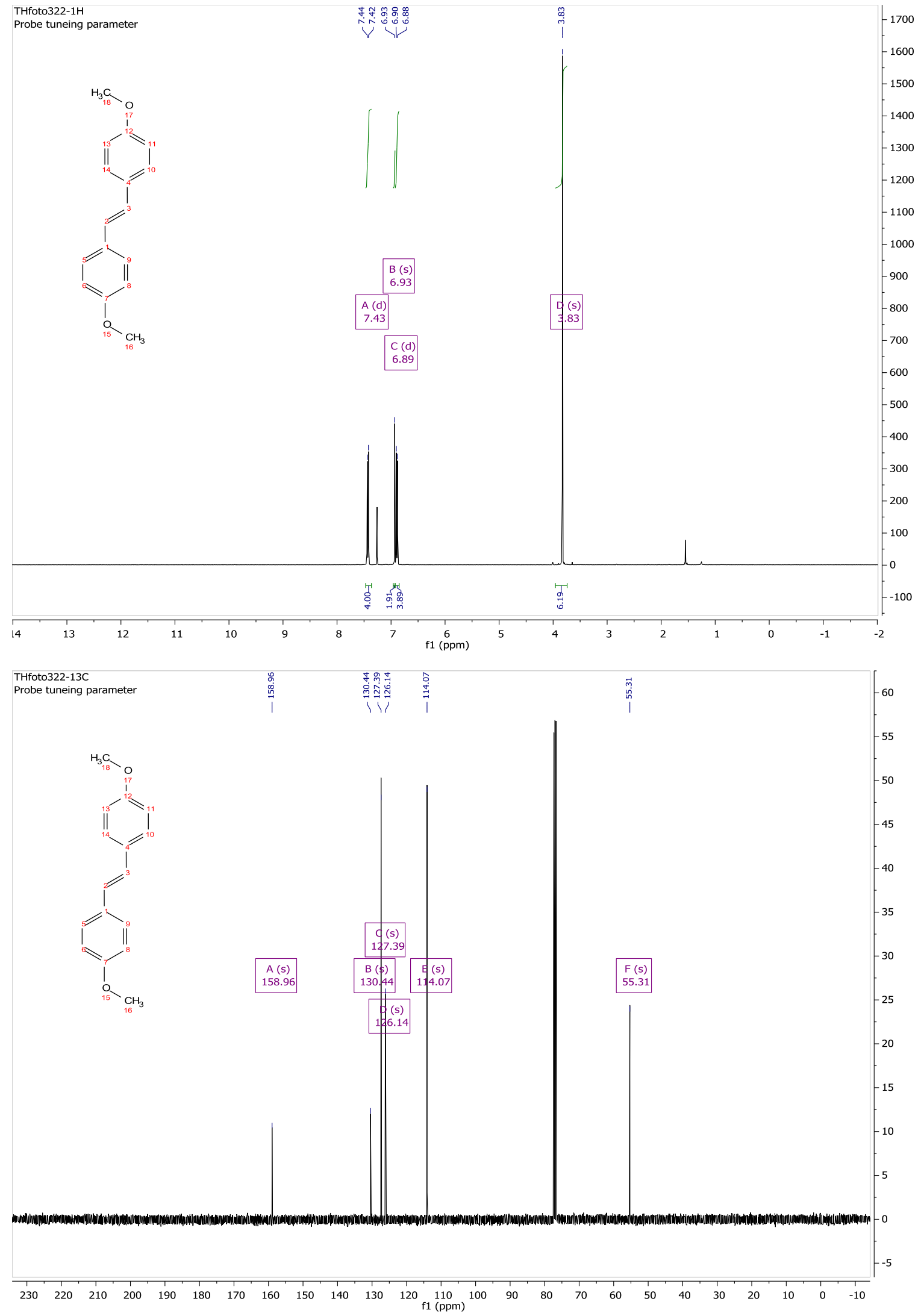
4j
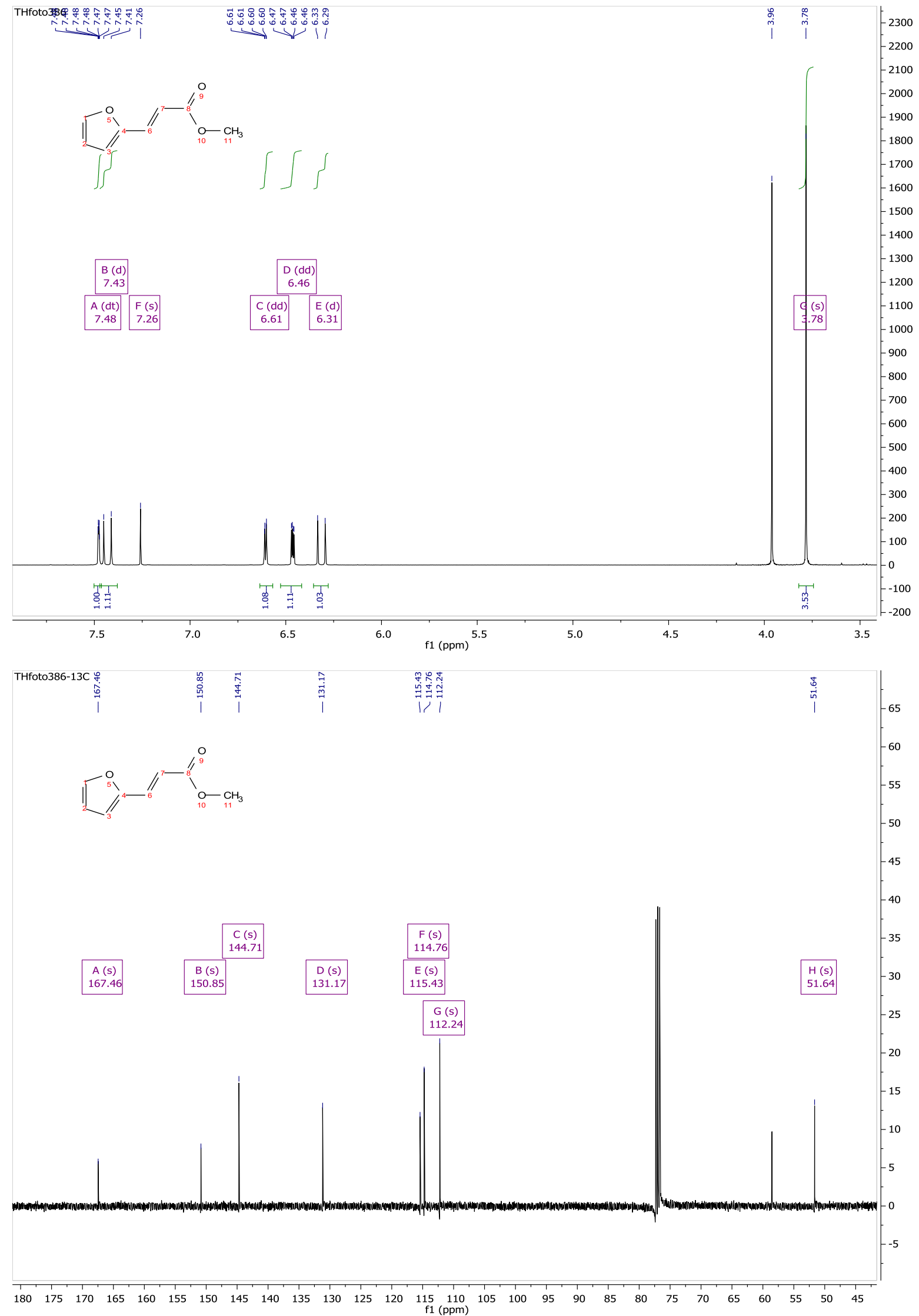
S14 Copy of NMR Spectra of compound 2c
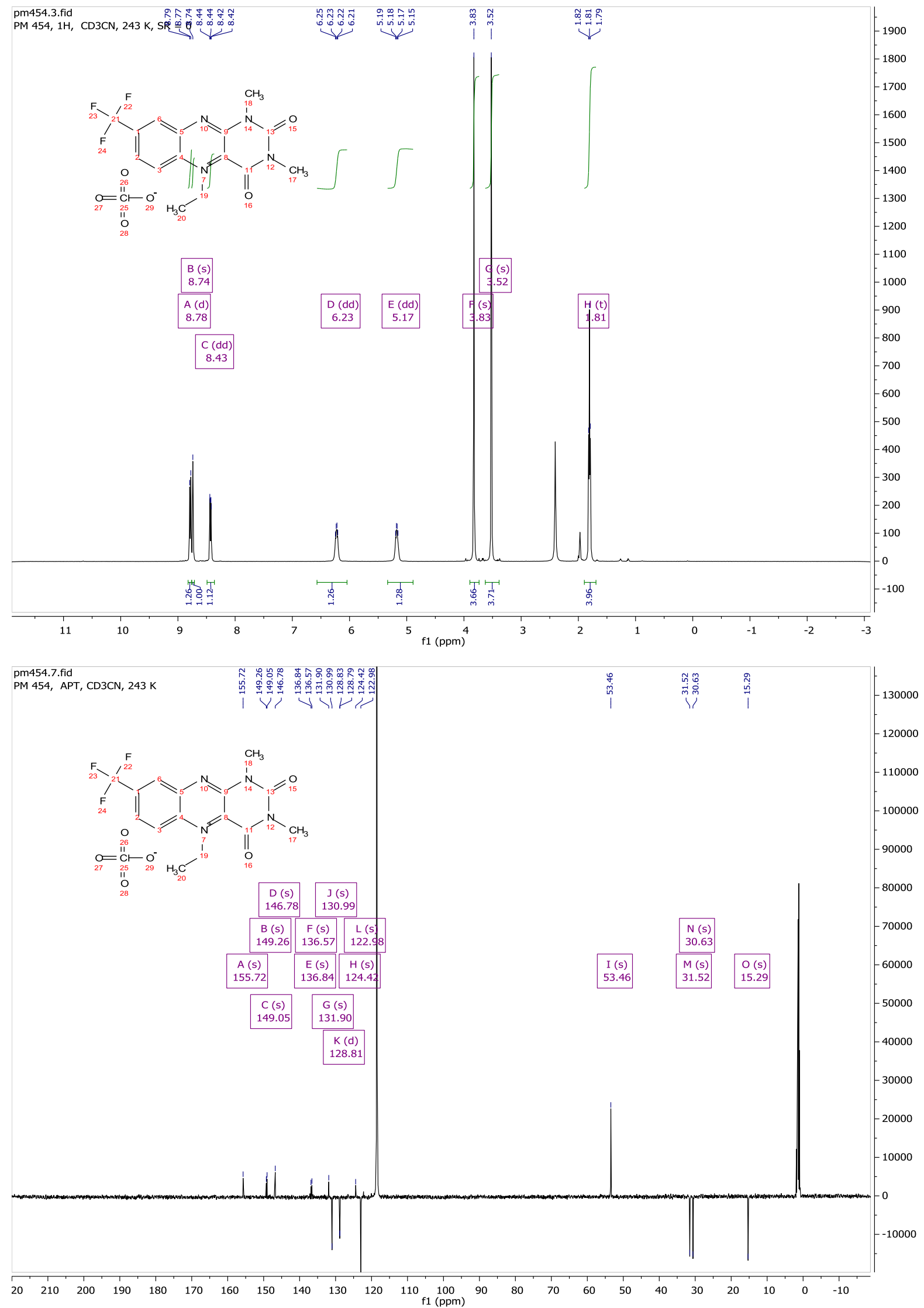